\title{
Palladium-Catalyzed Alkoxycarbonylation of Conjugated Enyne Oxiranes: A Diastereoselective Method for the Synthesis of 7-Hydroxy-2,3,5-trienoates
}

\author{
Melih Kuş, ${ }^{\dagger}$ Levent Artok, ${ }^{* \dagger}$ and Muhittin Aygün ${ }^{*}$ \\ ${ }^{\dagger}$ Department of Chemistry, Faculty of Science, Izmir Institute of Technology, Urla, 35430 Izmir, Turkey \\ ${ }^{\ddagger}$ Department of Physics, Faculty of Science, Dokuz Eylul University, Buca, Izmir, Turkey
}

Supporting Information

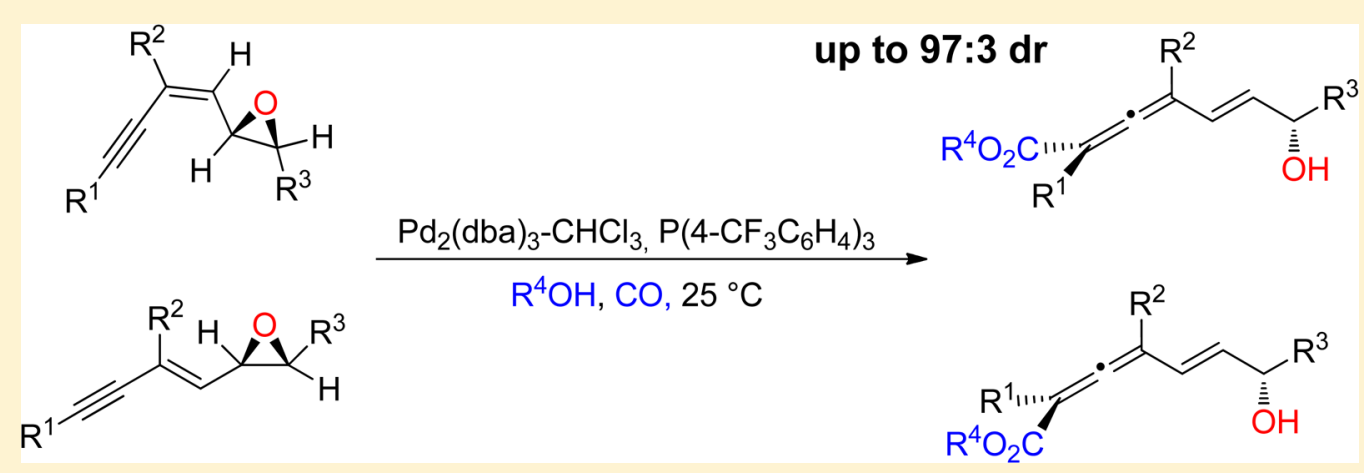

ABSTRACT: Palladium-catalyzed alkoxycarbonylative 1,5-substitution of conjugated enyne oxiranes provides a diastereoselective route to $(E)$-configured 7-hydroxy-2,3,5-trienoates. The reactions proceeded in a highly stereoselective manner, possibly through sequential formation of $\pi$-allylpalladium and $\sigma$-vinylallenyl palladium complexes. The major diastereomeric form of the product is determined by the configuration of the alkenyl moiety of the substrate.

\section{INTRODUCTION}

Vinylallenes are, interestingly, reactive compounds toward various cycloaddition and cyclization reactions. ${ }^{1}$ They exhibit particularly higher activity and selectivity with Diels-Alder reactions, because their configurational equilibrium is more on the side of s-cis conformer-a prerequisite for a $[4+2]$ cycloaddition reaction to occur effectively-than for 1,3 -dienes. ${ }^{2}$ In spite of their synthetic utility in organic reactions, there are only a few methods that can generate vinylallene structures. ${ }^{1 \mathrm{j}, \mathrm{o}, \mathrm{r}, 3}$

Recently, we introduced new methods for the construction of functionalized vinylallenes, which involved palladium- or rhodium-catalyzed reactions of carbonate or acetate derivatives of 2-en-4-yne alcohols. ${ }^{4}$ 2,3,5-Trienoates were a form of the vinylallene structure that was accomplished via palladiumcatalyzed alkoxycarbonylation of 2,4-enyne carbonates (Scheme 1). ${ }^{4 a}$

Scheme 1. Previous Study: Alkoxycarbonylation of Enyne Carbonates $^{4 a, c}$

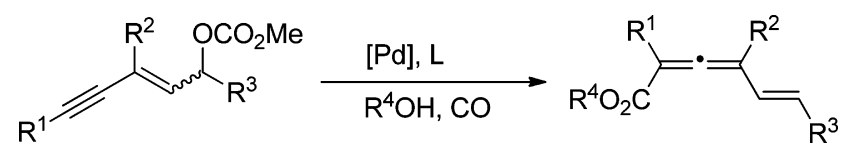

It was later found that in some cases, this reaction proceeds with high center-to-axial chirality transfer. ${ }^{4 c}$
Encouraged by these findings, we have decided to extend the methodology to enyne oxiranes in order to obtain more functionalized vinylallene structures. To the best of our knowledge, the only coupling method that involved the use of enyne oxiranes was their reactions with organocuprates, which led to alkyl-substituted vinylallenes that bear a hydroxyl group in the allylic position (Scheme 2). ${ }^{3 e}$ However, this

Scheme 2. Previous Study: 1,5- $\left(\mathrm{S}_{\mathrm{N}} 2^{\prime \prime}\right)$-Substitution Reactions of Enyne Oxiranes with Organocuprates ${ }^{3 e}$

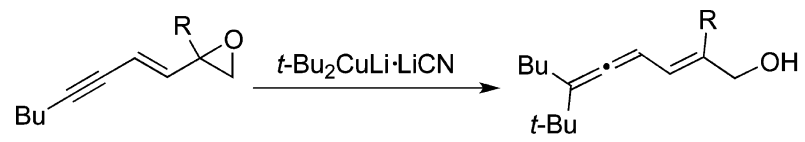

Only $(E)$-isomer when $\mathrm{R}$ is $\mathrm{H}$, whereas $(E) /(Z): 4: 1$ when $\mathrm{R}$ is $\mathrm{Me}$

method provided a very limited scope for both enyne substrates and their reacting partners, and moreover, the diastereoselectivity of this method, in terms of relative configuration of allenyl moiety and allylic carbon, could not be probed.

We describe here for the first time that conjugated enynes carrying an oxirane moiety as a leaving group undergo

Received: February 17, 2015

Published: April 26, 2015 
Table 1. Methoxycarbonylation of Enyne Oxirane $(Z)-1 a^{a}$<smiles>CC(C#CBr)=CC1OC1(C)C</smiles>

$(Z)-1 a$<smiles>COC(=O)C(Br)=C(C)C=CC(C)(C)O</smiles>

2aa

\begin{tabular}{|c|c|c|c|c|}
\hline entry & $\mathrm{Pd} \%$ & $\mathrm{PPh}_{3} \%$ & $t(\mathrm{~h})$ & yield $\%^{b}$ \\
\hline 1 & 0.5 & 2 & 1 & $>99^{c}$ \\
\hline 2 & 0.25 & 1 & 2 & $>99$ \\
\hline 3 & 0.5 & 3 & 3 & $>99$ \\
\hline 4 & 0.25 & 0.5 & 5 & $>99$ \\
\hline 5 & 1 & 0 & 16 & 0 \\
\hline 6 & 0 & 4 & 16 & 0 \\
\hline
\end{tabular}

${ }^{a}$ Reaction conditions: $0.3 \mathrm{mmol}$ of (Z)-1a, $2 \mathrm{~mL}$ of $\mathrm{MeOH}$, under CO filled balloon, $25{ }^{\circ} \mathrm{C} .{ }^{b}$ Determined by ${ }^{1} \mathrm{H}$ NMR analysis using benzaldehyde as the internal standard. ${ }^{c}$ Isolated yield.

Table 2. Effect of Ligand on Methoxycarbonylation of $(\mathrm{Z})-1 \mathrm{~b}^{a}$

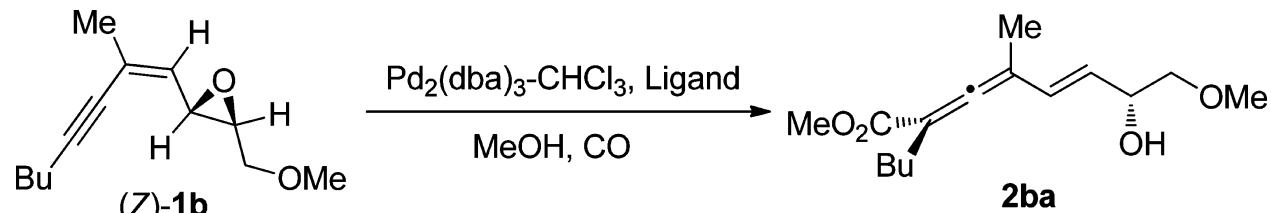

\begin{tabular}{|c|c|c|c|c|}
\hline entry & ligand & $t(\mathrm{~h})$ & yield $\%^{b}$ & $\mathrm{dr}^{c}$ \\
\hline 1 & $\mathrm{PPh}_{3}$ & 1 & 96 & $92: 8$ \\
\hline 2 & $\mathrm{PPh}_{3}{ }^{d}$ & 4 & 89 & $92: 8$ \\
\hline 3 & $\mathrm{P}\left(2-\mathrm{MeC}_{6} \mathrm{H}_{4}\right)_{3}$ & 0.5 & 92 & $92: 8$ \\
\hline 4 & $\mathrm{P}\left(2-\mathrm{OMeC}_{6} \mathrm{H}_{4}\right)_{3}$ & 0.5 & 97 & $87: 13$ \\
\hline 5 & $\mathrm{P}\left(4-\mathrm{OMeC}_{6} \mathrm{H}_{4}\right)_{3}$ & 1 & 95 & $86: 14$ \\
\hline 6 & $\mathrm{PPh}_{2} \mathrm{Me}$ & 2.5 & 81 & $80: 20$ \\
\hline 7 & Tri(2-furyl)phosphine & 1 & 94 & $83: 17$ \\
\hline 8 & $\mathrm{P}\left(4-\mathrm{CF}_{3} \mathrm{C}_{6} \mathrm{H}_{4}\right)_{3}$ & 0.5 & $94^{e}$ & $94: 6$ \\
\hline 9 & dppf & 1 & 72 & $90: 10$ \\
\hline 10 & dppe & 3 & 96 & $86: 14$ \\
\hline 11 & dppb & 1.5 & 89 & $86: 14$ \\
\hline 12 & Xantphos & 1.5 & 92 & $86: 14$ \\
\hline 13 & $( \pm)$-BINAP & 9.5 & $44^{f}$ & $85: 15$ \\
\hline 14 & BIPHEP & 10 & 0 & \\
\hline
\end{tabular}

${ }^{a}$ Reaction conditions: $0.1 \mathrm{mmol}$ of $(Z)-\mathbf{1 b}, 2 \mathrm{~mL}$ of $\mathrm{MeOH}, 1 \mathrm{~mol} \%$ of $\mathrm{Pd}, \mathrm{P} / \mathrm{Pd}$ ratio is 4:1, under CO-filled balloon, $25^{\circ} \mathrm{C}$. dppf: $1,1^{\prime}$ bis(diphenylphosphino)ferrocene; dppe: 1,2-bis(diphenylphosphino)ethane; dppb: 1,4-bis(diphenylphosphino)butane; Xanthphos: 4,5-bis(diphenylphosphino)-9,9-dimethylxanthene; BINAP: 2,2'-bis(diphenylphosphino)-1,1'-binaphthalene; BIPHEP: 2,2'-bis(diphenylphosphino)-1,1'biphenyl. ${ }^{b}$ Determined by ${ }^{1} \mathrm{H}$ NMR analysis using benzaldehyde as the internal standard. ${ }^{c}$ Diastereomeric ratio (dr) was determined by ${ }^{1} \mathrm{H}$ NMR. ${ }^{d} \mathrm{Pd}\left(\mathrm{PPh}_{3}\right)_{4}$ was the catalyst of this reaction. ${ }^{e}$ Isolated yield. ${ }^{f}$ The conversion of $(\mathrm{Z})-\mathbf{1 b}$ was $49 \%$.

alkoxycarbonylation in the presence of palladium under fairly mild conditions, forming hydroxy-functionalized 2,3,5-trienoates usually with high diastereoselectivities.

\section{RESULTS AND DISCUSSION}

We initiated our study using the enyne oxirane (Z)-1a whose oxirane terminus is dimethyl-substituted. We were pleased to observe that, with just our first attempt, the substrate $(Z)$-1a was converted to the desired 2,3,5-trienoate product 2aa, which is exclusively in $(E)$-configuration, and carries a hydroxyl group on the allylic position, almost quantitatively under very mild reaction conditions; the conversion was complete within just $1 \mathrm{~h}$, when the reaction was performed in the presence of a $\mathrm{Pd}_{2} \mathrm{dba}_{3}-\mathrm{CHCl}_{3}$ (0.5 mol \% Pd) and $\mathrm{PPh}_{3}$ (2 mol \%) combination in $\mathrm{MeOH}$, and under balloon pressure of $\mathrm{CO}$ at $25{ }^{\circ} \mathrm{C}$ (Table 1 , entry 1 ). The catalyst loading could even be reduced by half $\left(0.25 \% \mathrm{Pd}, 1 \% \mathrm{PPh}_{3}\right)$ without compromising the product yield (entry 2 ). The optimum $\mathrm{PPh}_{3} / \mathrm{Pd}$ ratio appears to be $4: 1$, because any deviation from this ratio resulted in a decrease in the reaction rate (entries 1-4). Finally, the controlled experiments proved that no conversion for the substrate would be possible in the absence of a phosphine ligand or a palladium source (entries 5 and 6).

Having determined that the enyne oxirane ( $Z$ )-1a is a perfectly amenable reagent toward palladium catalysis as a means to synthesize vinylallene structures, we next probed the diastereoselectivity-directly associated with the level of center-to-axial chirality transfer-of the method over the substrate $(Z)-\mathbf{l b}$, using a variety of mono- and bidentate ligands. The corresponding vinylallene product 2 ba was in $96 \%$ yield with a good diastereomeric ratio (92:8) when $\mathrm{PPh}_{3}$ was used as the ligand (Table 2, entry 1 ). The relative configuration of its 
Table 3. Alkoxycarbonylation of Enyne Oxirane $(Z)-1 a^{a}$

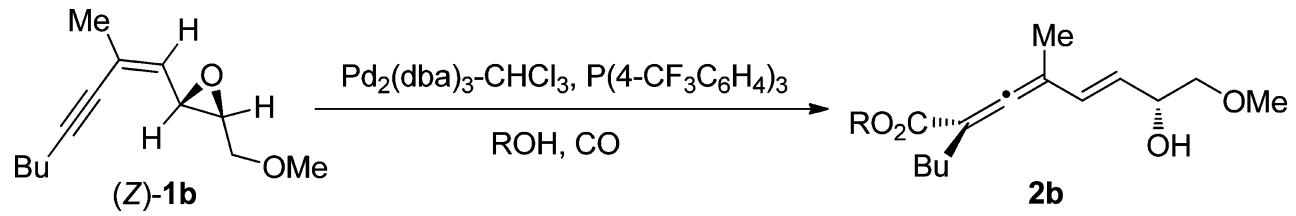

$(Z)-1 b$

$\begin{array}{cc}\text { entry } & \mathrm{ROH} \\ 1 & \mathrm{EtOH} \\ 2 & \mathrm{PrOH} \\ 3 & \mathrm{BuOH} \\ 4 & i-\mathrm{PrOH} \\ 5 & t \text {-BuOH } \\ 6 & \text { allyl alcohol } \\ 7^{d} & \end{array}$

$\begin{array}{cr}\text { product } & t(\mathrm{~h}) \\ \mathbf{2 b b} & 1 \\ \mathbf{2 b c} & 3 \\ \mathbf{2 b d} & 4 \\ \mathbf{2 b e} & 2 \\ \mathbf{2 b f} & 10 \\ \mathbf{2 b g} & 6 \\ & 1\end{array}$

$\begin{array}{rr}\text { (h) } & \text { yiel } \\ 1 & 84 \\ 3 & 87 \\ 4 & 83 \\ 2 & 88 \\ 10 & \\ 6 & 25 \\ 1 & 91\end{array}$

$\begin{array}{cc}\text { yield \% } & \mathrm{dr}^{b} \\ 84 & 80: 20 \\ 87 & 88: 12 \\ 83 & 86: 14 \\ 88 & 86: 14 \\ 0 & - \\ 25^{c} & 93: 7 \\ 91 & 93: 7\end{array}$

${ }^{a}$ Performed under the conditions of Table $2 .{ }^{b}$ Determined by ${ }^{1} \mathrm{H}$ NMR. ${ }^{c}$ The conversion of $(\mathrm{Z})-\mathbf{1 b}$ was $75 \%$. ${ }^{d}$ Reaction was performed with $3 \mathrm{~mol} \%$ of $\mathrm{Pd}$ and $12 \mathrm{~mol} \%$ of ligand.

Scheme 3. Methoxycarbonylation of (E)-1b

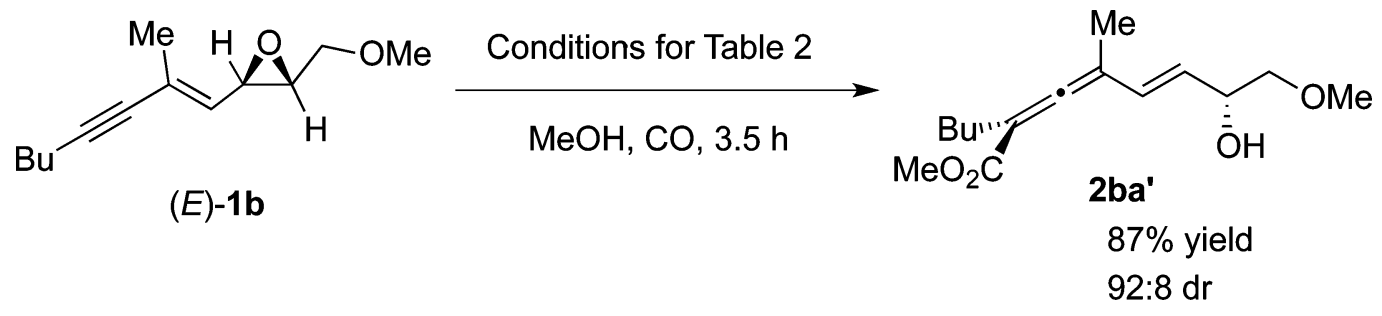

major diastereomer was determined by X-ray crystallography of its phenylcarbamate derivative (see the Supporting Information). ${ }^{5}$

Typically, comparable yields are obtained with a variety of monodentate phosphine ligands tested herein, with varying reaction times required for a full conversion. However, it has been found that the level of the chirality transfer is strongly dependent on the ligand type that is used. The reactions in the presence of a prepared catalyst $\mathrm{Pd}\left(\mathrm{PPh}_{3}\right)_{4}$ or $\mathrm{P}\left(2-\mathrm{MeC}_{6} \mathrm{H}_{4}\right)_{3}$ ligand proceeded with similar selectivities when compared with $\mathrm{PPh}_{3}$ (entries 2 and 3), whereas electron-rich phosphines and a heteroaryl-substituted phosphine all showed inferior stereoselectivities (entries 4-7). The best diastereoselectivity was obtained with the use of an electron-poor phosphine ligand, $\mathrm{P}\left(4-\mathrm{CF}_{3} \mathrm{C}_{6} \mathrm{H}_{4}\right)_{3}$, which afforded 2 ba with $94 \%$ yield and 94:6 dr within just $30 \mathrm{~min}$ (entry 8). Finally, the use of bidentate ligands brought about dismal selectivities or low activities in some cases (entries 9-14).

Having determined $\mathrm{P}\left(4-\mathrm{CF}_{3} \mathrm{C}_{6} \mathrm{H}_{4}\right)_{3}$ as the optimal ligand, next we explored the suitability of a number of alcohol reagents for their reactions with $(Z)-\mathbf{1 b}$. Except those with $t-\mathrm{BuOH}$ (Table 3, entry 5), the vinylallene products could be obtained at yields within the range of $83-91 \%$ with all alcohol reagents tested herein. Surprisingly, however, the reactions performed with alkyl alcohols, including $\mathrm{EtOH}, \mathrm{PrOH}, \mathrm{BuOH}$, and $i-\mathrm{PrOH}$, all proceeded with relatively lower stereoselectivities (entries 1-5). Nevertheless, gratifyingly an additional functionality could be introduced to a 7-hydroxy-2,3,5-trienoate structure with the use of allyl alcohol, which yielded the corresponding product $\mathbf{2 b g}$ in $91 \%$ yield and with 93:7 dr, albeit with higher Pd loading to complete the reaction (entries 6-7).

It seems that the method is also suitable for enyne oxiranes containing an $(E)$-configured alkenyl moiety; a carbonylation reaction of $(E)-\mathbf{1 b}$ in $\mathrm{MeOH}$ under the standard conditions (conditions denoted in Table 2) required remarkably longer reaction period for complete conversion and resulted in a diastereomer of $\mathbf{2} \mathbf{b a}\left(\mathbf{2} \mathbf{b a}^{\prime}\right)$ with a high yield, but slightly decreased selectivity of 92:8 dr (Scheme 3). This result also proves that the configuration of alkenyl moiety of substrate regulates the stereomeric outcome of the product. Nonetheless, because of some difficulties encountered during the synthesis of enyne oxiranes with (E)-alkenyl group, such as partial isomerization of the alkenyl group, further studies were continued using only $(Z)$-configured reagents.

Next we explored the scope of the protocol over a variety of substrates containing $(Z)$-alkenyl and $(E)$-oxirane moieties. Except in one case, alkoxycarbonylation reactions proceeded rather smoothly and provided the expected products at yields within the range of $81-92 \%$.

It was interesting to observe that the form of pendant oxygen functionality within $\mathrm{R}^{3}$ group is an important factor for the stereoselectivity of the process. Substituting the methoxymethyl group in $\mathrm{R}^{3}$ position with a benzyloxymethyl group $((Z)-1 \mathrm{c})$ had no detectable effect on both the reactivity and stereoselectivity (Table 4, entry 1 ). However, when $\mathrm{R}^{3}$ was a carbinol group $((Z)-1 \mathrm{~d})$ or its hydroxyl group was silyl-protected $((Z)-1 \mathbf{e})$, the corresponding methoxycarbonylated products 2da and 2ea were obtained with 90:10 and 89:11 dr, respectively (entries 2 and 3 ).

The same trend was also obvious for the reactions of $(Z)$-1f and $(Z)-\mathbf{1 g}$, where $\mathrm{R}^{3}$ is dimethylcarbinol and dimethylmethoxy methyl, respectively. Though comparably good yields could be achieved with both reagents, the products $2 \mathrm{fa}$ and $\mathbf{2 g a}$ were obtained with significantly different level of stereoselectivities, 85:15 and 95:5 dr, respectively (entries 4 and 5).

It seems that for an effective stereoselectivity, the alkenyl carbon that is proximal to the alkynyl moiety is required to be fully substituted, because the carbonylative reaction of the enyne oxirane containing a disubstituted alkenyl moiety $((Z)-\mathbf{1 h})$ with 
Table 4. Alkoxycarbonylation of Enyne Oxiranes ${ }^{a}$

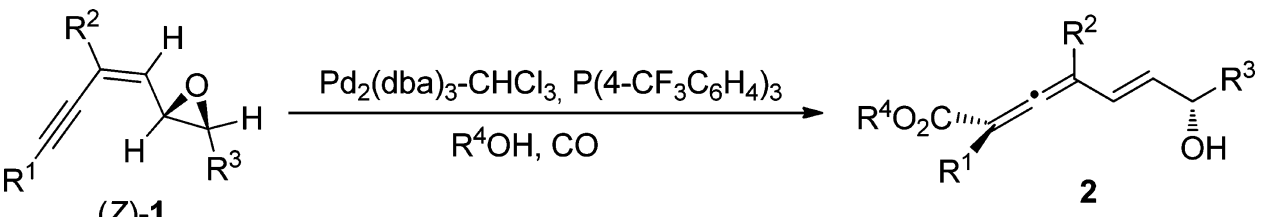

\section{$(Z)-1$}

\begin{tabular}{llll}
\hline entry product & $\begin{array}{l}\text { time } \\
(\min )\end{array}$ & $\begin{array}{l}\text { yield }^{b} \\
(\%)\end{array}$ & $\mathrm{dr}^{c}$ \\
\hline
\end{tabular}

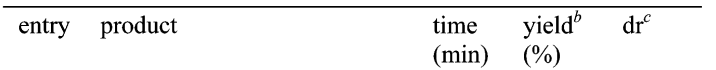

2<smiles>CC(=O)C(Br)=C(C)C=C[C@@H](O)CCl</smiles>

3<smiles>CCOCC(O)C=CC(C)=C(Br)C(C)=O</smiles>

4<smiles>CCCC(=CC(C)=CC=CC(C)(C)O)C(C)(C)O</smiles>

5<smiles>CO[C@H](Br)/C=C(\C)C=C[C@H](O)C(C)(C)O[Na]</smiles>

6<smiles>COC[C@H](O)/C=C/C=C/C(=O)OC</smiles>

7<smiles>CC(=O)C(Br)=C(Br)C=CC(O)C(C)(Br)Br</smiles>

8<smiles>CC(=O)C(Br)=C(C)C=CC(C)O</smiles>

9<smiles>COC[C@H](O)/C=C/C(Cl)=C(/Br)C(C)Br</smiles>

10

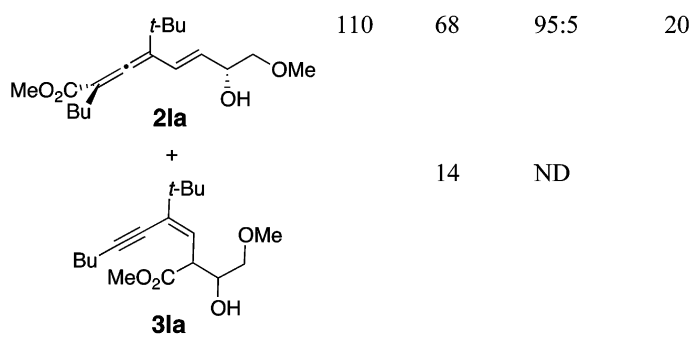

$\mathrm{MeO}_{2} \mathrm{C}$<smiles>COC[C@H](O)/C=C/C(C)=C1/[C@@H]([14CH3])[C@@H]1OC</smiles>

13<smiles></smiles>

$85: 15 \quad 14$
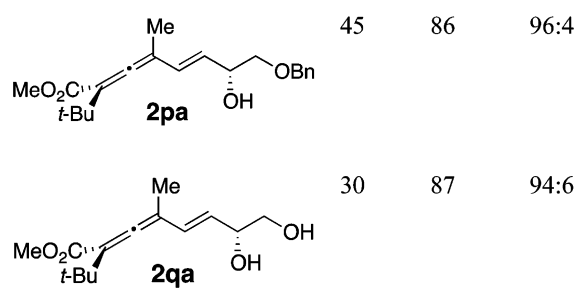

16

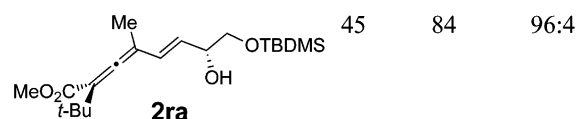

17

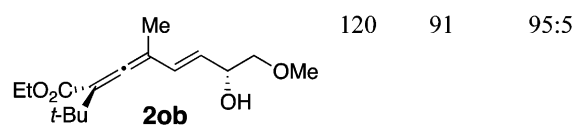

18

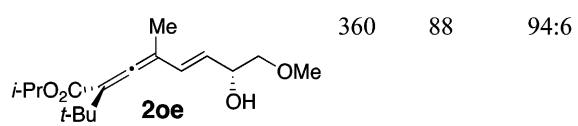

$19^{c}$
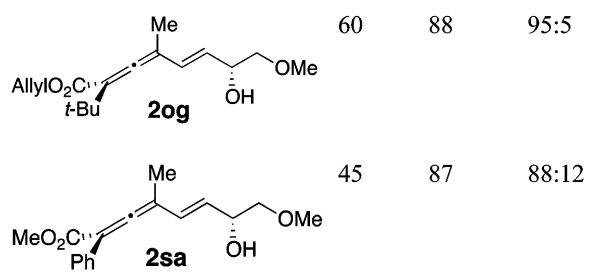

${ }^{a}$ Performed under the conditions of Table $2 .{ }^{b}$ Determined by ${ }^{1} \mathrm{H}$ NMR. ${ }^{c}$ Reaction was performed with 3 mol $\%$ of Pd and 12 mol $\%$ of ligand.

$\mathrm{MeOH}$ proceeded with an inferior stereoselectivity (entry 6). As a model for substrates with all alkyl substituents, the enyne oxirane $(Z)-1 \mathrm{i}$, where all $\mathrm{R}^{1}, \mathrm{R}^{2}$, and $\mathrm{R}^{3}$ positions were occupied by butyl groups, also gave rise to the expected product $2 \mathrm{ia}$ with a high selectivity of $93: 7 \mathrm{dr}$ (entry 7$)$. However, $(Z)-\mathbf{1 j}$ possessing a methyl group in the $\mathrm{R}^{3}$ interestingly afforded the lowest $\mathrm{dr}(82: 18)$ as compared to other enyne oxiranes tested herein (entry 8$){ }^{6}$ While the method tolerated a larger cyclohexyl 
group in the $\mathrm{R}^{2}$ (entry 9) under the reaction conditions, the presence of the tert-butyl group at this position $((Z)-11)$ brought about the formation of an allylic esterified byproduct (3la) along with the desired product 2la with a high $\mathrm{dr}$ (entry 10). ${ }^{7}$

It was also intriguing to find that there appears to be a direct relation between the diastereoselectivity and the size of $\mathrm{R}^{1}$ (entries $11-13) .{ }^{8}$ For instance, when $(Z)-1 \mathbf{m}$, which contains a terminal alkynyl moiety, was subjected to the methoxycarbonylation, the product $2 \mathrm{ma}$ was obtained with only $88: 12$ dr. Nevertheless, the substrate $(Z)-10\left(\mathrm{R}^{1}=t-\mathrm{Bu}\right)$ afforded the expected product 2oa in an excellent level of selectivity (97:3 dr).

The remarkable improvement on the stereoselectivity of the process that was gained with the presence of the tert-butyl group on the alkynyl moiety appears enduring; in contrast to the results obtained with $(Z)-1 \mathbf{b}$, when $\mathrm{R}^{1}=$ tert-butyl, the replacement of the methoxy group with benzyloxy- $((Z)-\mathbf{1 p})$, hydroxyl- $((Z)-\mathbf{1 q})$, or silyl-protected $((Z)-\mathbf{1 r})$ groups within $\mathrm{R}^{3}$ only slightly impacted the $\mathrm{dr}$ of the corresponding products (entries 14-16). Moreover, the carbonylative reaction of (Z)-1o with other alcohols also provided the corresponding vinylallenes with high dr values (entries 17-19).

The enyne oxirane ( $Z$ )-1s with a phenyl substituent at alkynyl terminus yielded the product 2 sa with 88:12 dr (entry 20), and the substrate 1t having an endocyclic double bond also successfully underwent methoxycarbonylation to generate an exocyclic conjugated vinylallene structure 2 ta with a relatively lower but synthetically still useful dr (Scheme 4).

\section{Scheme 4. Methoxycarbonylation of $1 \mathrm{t}$}

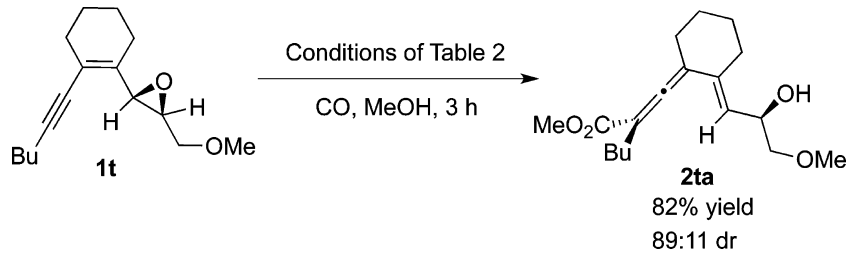

The mechanisms illustrated in Scheme 5 are proposed to account for the stereoselective formation of $\mathbf{2}$ and $\mathbf{2}^{\prime} .{ }^{9}$ The reaction cycle should begin with ring-opening by the attack of a palladium complex to both $(Z)-1$ and $(E)-1$ in anti-mode, ${ }^{10}$ leading to the formation of $\pi$-allylpalladium complexes $\mathbf{A}$ and $\mathbf{A}^{\prime}$, respectively, ${ }^{10 \mathrm{~d}, 11}$ Given the formation of vinylallenes exclusively in $(E)$-configuration for both intermediates ( $\mathbf{A}$ and $\left.\mathbf{A}^{\prime}\right)$, the alkanolate group $\left(\mathrm{CHR}^{3} \mathrm{O}^{-}\right)$on the planar allyl ligand should be oriented syn with respect to the middle allylic $\mathrm{C}-\mathrm{H}$. These intermediates additionally undergo a palladium shift to the far alkynyl carbon proceeding mainly with retention to form vinylallenyl palladium complexes $\mathbf{B}$ and $\mathbf{B}^{\prime}$, respectively. The relative position of the $\mathrm{R}^{2}$ group and alkynyl moiety on $\mathbf{A}$ and $\mathbf{A}^{\prime}$ may also be inverted via the $\pi-\sigma-\pi$ interconversion into $\mathbf{C}$ and $\mathbf{C}^{\prime},{ }^{4 c, 10 c}$ respectively, before undergoing such a $\mathrm{Pd}$ shift. The favored configuration of this side of the complex should be regulated by the inherent structure of $R^{2}$. For instance, when $R_{2}$ is hydrogen the thermodynamically more stable $\pi$-allyl complexes should bear alkynyl and $\mathrm{CHR}^{3} \mathrm{O}^{-}$moieties in syn,syn positions $\left(\mathbf{A}^{\prime}\right.$ and $\left.\mathbf{C}\right) .^{10 c, d, 11}$

However, such configurational transitions cannot alter the outcome of the reaction, because both isomeric intermediates $\mathbf{C}$ and $\mathbf{C}^{\prime}$ would also lead to $\mathbf{B}$ and $\mathbf{B}^{\prime}$, respectively. These $\sigma$-allenylpalladium complexes undergo successive $\mathrm{CO}$ insertion and reductive elimination, as verified previously, ${ }^{4,12}$ to yield 2 and $\mathbf{2}^{\prime}$ stereoselectively.
The loss of stereochemical integrity of the resulting products should take place during the course of the reaction cycle because subjecting the purified $\mathbf{2} \mathbf{b a}$ again to the standard conditions for $4 \mathrm{~h}$ had no influence on its original diastereomeric ratio. ${ }^{13}$ Though we cannot completely disregard the occurrence of the $\pi$-allylpalladium isomerization or its formation through synattack of the palladium, the back side migration of the palladium over the alkynyl side (by inversion of configuration) could be the primary basis for the formation of the minor diastereomeric form in each case, and particularly increased sizes of $R^{1}$ and $R^{2}$ might have limited the occurrence of such transitions in an inverted manner. However, the presence of a highly encumbered group, such as tert-butyl, at $\mathrm{R}^{2}$ also retards the effective migration of the $\pi$-allyl coordinated palladium, and as a result ( $Z$ )-11 partly underwent allylic alkoxycarbonylation to form the byproduct 3la.

Finally, the enyne oxirane $(Z)-1 \mathbf{i}^{\prime}$ containing a $(Z)$-configured oxirane moiety was also subjected to the standard reaction conditions. The reaction proceeded smoothly in accordance to the proposed mechanism, and thus yielded the vinylallene product $2 \mathbf{i a}^{\prime}$, which is the diastereomer of $2 \mathbf{i a}$ (see entry 7 of Table 4), with a high level of $\mathrm{dr}$ (Scheme 6).

\section{CONCLUSION}

In summary, we have established an unprecedented, mild, and efficient palladium-catalyzed diastereoselective 1,5-type alkoxycarbonylative substitution over conjugated enyne oxiranes, which provides a highly selective route to anti-substituted (E)-configured vinylallenes bearing a hydroxyl group on the allylic position. The reactions proceeded through successive formation of zwitterionic $\pi$-allyl- and $\sigma$-allenylpalladium complexes.

\section{EXPERIMENTAL SECTION}

General Methods. THF, DMF, and DCM solvents were all purified by a solvent purification system. $\mathrm{Et}_{2} \mathrm{O}$ was distilled from benzophenone-ketyl under argon prior to use. Methanol and ethanol were dried over $\mathrm{Mg}$ turnings in the presence of iodine and stored on molecular sieve $3 \AA$ under Ar. 1-Propanol and 2-propanol were dried first by stirring over anhydrous $\mathrm{CaO}$ followed by refluxing over $\mathrm{Mg}$ turnings in the presence of iodine. 1-Butanol was dried first by stirring over anhydrous $\mathrm{MgSO}_{4}$ and then refluxed over $\mathrm{Mg}$ turnings in the presence of iodine. tert- $\mathrm{BuOH}$ was stirred over $\mathrm{CaH}_{2}(5 \% \mathrm{w} / \mathrm{v})$, distilled, and stored on molecular sieve $3 \AA$ under Ar.

Syntheses of all enyne oxirane starting materials ( 1 and $\left.\mathbf{1}^{\prime}\right)$ were performed under Ar gas, and purification of all synthesized molecules was performed by column chromatography on silica gel. Silica gel material used for the purification of enyne oxirane substrates had a particle size range of $60-200$ mesh and was treated by $\mathrm{NEt}_{3}$ before use. It must be noted that the column chromatography of the substrate $\mathbf{1}$ and $\mathbf{1}^{\prime}$ on an untreated silica gel always resulted in decomposition. All other column purifications were performed on silica gel $60(35-70 \mu \mathrm{m})$. All substrates and vinylallene products appeared either colorless or as pale yellow oils.

The synthesized reactants and carbonylation products were analyzed by GC and GC-MS. NMR spectra were recorded on a $400 \mathrm{MHz}$ spectrometer. Chemical shifts are reported in ppm downfield from $\mathrm{Me}_{4} \mathrm{Si}$. Infrared spectra were obtained by ATR method with neat samples. High-resolution mass spectral analyses of new compounds were performed using EI-High Resolution Double Focusing Magnetic Sector (ionization mode: $70 \mathrm{eV}$, emission current: $1 \mathrm{~mA}$, source temperature: $160{ }^{\circ} \mathrm{C}$, resolution: 10000 (10\% valley definition)) and ESI-LTQ Orbitrap (source voltage: $+3.8 \mathrm{kV}$, capillary voltage: $41 \mathrm{~V}$, capillary temperature: $275^{\circ} \mathrm{C}$, tube lens voltage: $140 \mathrm{~V}$, system resolution: 60000 (10\% valley definition)).

The $\mathrm{Pd}_{2}(\mathrm{dba})_{3}-\mathrm{CHCl}_{3}$ complex was synthesized in the laboratory. ${ }^{14}$

Synthesis of (Z)-1i and (Z)-1i'. 1-Hexyne $(2.3 \mathrm{~mL}, 20 \mathrm{mmol})$ was added dropwise to a solution of $\mathrm{BBr}_{3}(22 \mathrm{~mL}, 22 \mathrm{mmol}, 1 \mathrm{M})$ in dry 
Scheme 5. Mechanism of Alkoxycarbonylation of Conjugated Enyne Oxiranes

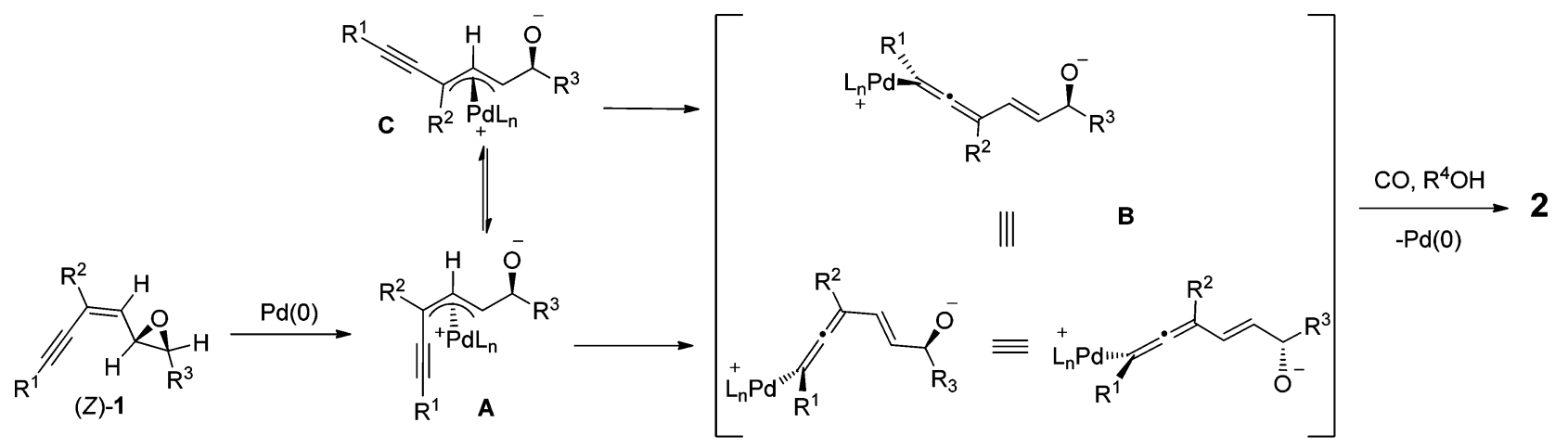

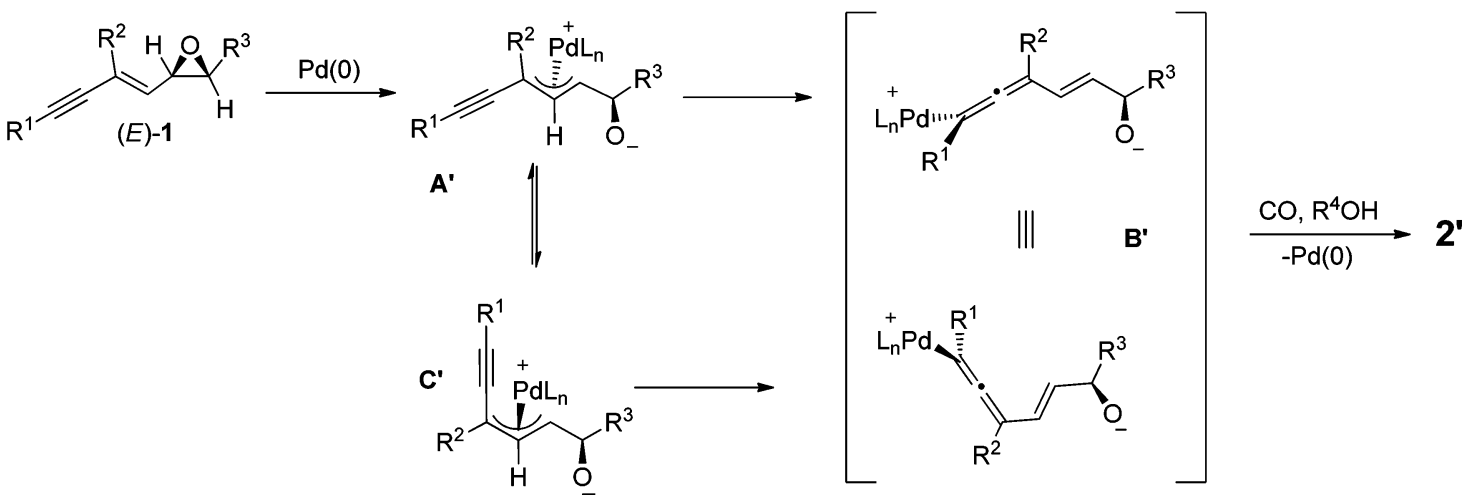

Scheme 6. Methoxycarbonylation of $(Z)-1 i^{\prime}$

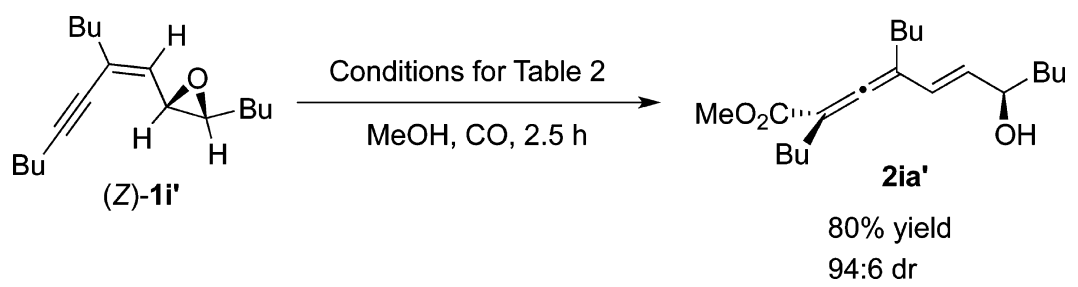

DCM at $-78{ }^{\circ} \mathrm{C}$. The mixture was allowed to warm to $0{ }^{\circ} \mathrm{C}$ and stirred for $1 \mathrm{~h}$. The reaction mixture was recooled to $-78{ }^{\circ} \mathrm{C}$, and a solution of 2,3-dimethylbutan-2,3-diol (2.84g, $24 \mathrm{mmol})$ in dry DCM $(20 \mathrm{~mL})$ was added dropwise. Following this addition, the mixture was warmed to ambient temperature and stirred for $1 \mathrm{~h}$. Then, the mixture was quenched by brine and extracted with DCM. The combined organic layers were dried over $\mathrm{MgSO}_{4}$, filtered, and evaporated. The residue was purified on silica gel column to afford $\mathbf{S} 1$ as a colorless oil (hexane-EtAc, yield: $3.57 \mathrm{~g}, 62 \%) .{ }^{15}$

To a stirred solution of $\mathrm{S} 1(4.64 \mathrm{~g}, 16 \mathrm{mmol})$ and $\mathrm{PdCl}_{2}\left(\mathrm{PPh}_{3}\right)_{2}$ $(1 \mathrm{~mol} \%, 112 \mathrm{mg}, 0.16 \mathrm{mmol})$ in dry THF $(20 \mathrm{~mL})$ was added a solution of 1-hexynylzinc chloride, which was generated by treatment of 1-hexyne (1.6 g, $19.2 \mathrm{mmol})$ with BuLi $(8.5 \mathrm{~mL}, 20.7 \mathrm{mmol}, 2.5 \mathrm{M}$ in hexane) in dry THF $(20 \mathrm{~mL})$ for $30 \mathrm{~min}$ at $-78{ }^{\circ} \mathrm{C}$ and following treatment with $\mathrm{ZnCl}_{2}(2.72 \mathrm{~g}, 19.2 \mathrm{mmol})$ for $30 \mathrm{~min}$ at $0{ }^{\circ} \mathrm{C}$. The resultant reaction mixture was stirred at room temperature (rt) for $1 \mathrm{~h}$ before quenching by $0.5 \mathrm{M} \mathrm{HCl}(\mathrm{aq})$. The content of the reaction medium was extracted with ether, washed with saturated $\mathrm{NaHCO}_{3}$ and brine, dried over $\mathrm{MgSO}_{4}$, filtered, and concentrated under reduced pressure. The residue was purified on silica gel column to afford $\mathbf{S 2}$ as a colorless oil (hexane-EtAc, yield: $3.9 \mathrm{~g}, 84 \%) .{ }^{15}$

A mixture of $1.5 \mathrm{~mol} \%$ of $\mathrm{Pd}_{2}(\mathrm{dba})_{3}(137 \mathrm{mg}, 0.15 \mathrm{mmol}), 6 \mathrm{~mol} \%$ of $\mathrm{AsPh}_{3}(184 \mathrm{mg}, 0.6 \mathrm{mmol})$, and $15 \mathrm{~mL}$ of $1 \mathrm{M} \mathrm{KOH}(\mathrm{aq})$ in $50 \mathrm{~mL}$ of THF was cooled to $0{ }^{\circ} \mathrm{C}$ before the addition of the compound $\mathbf{S 2}$ $(2.9 \mathrm{~g}, 10 \mathrm{mmol})$. Then, a THF solution $(10 \mathrm{~mL})$ of trans ${ }^{16}$ or cis-1iodo-1-hexene $\mathrm{i}^{17}(2.52 \mathrm{~g}, 12 \mathrm{mmol})$, which were synthesized according to the literature procedures, was added dropwise, and the reaction stirred for $30 \mathrm{~min}$ at room temperature. After the completion of the reaction, water was added to the resulting mixture before extracting with ether. The combined extracts were dried over $\mathrm{MgSO}_{4}$, filtered, and concentrated under reduced pressure. The residue was purified on silica gel column to afford the (5E,7Z)-8-butyltetradeca-5,7-dien-9-yne (S3i) or (5Z,7Z)-8-butyltetradeca-5,7-dien-9-yne (S3i') as yellowish oil products (hexane-EtAc, yield of S3i: $1.89 \mathrm{~g}, 77 \%$; yield of S3i': $1.25 \mathrm{~g}, 51 \%){ }^{18}$

To a solution of $\mathbf{S} 3 \mathbf{i}$ or $\mathbf{S} 3 \mathbf{i}^{\prime}(492 \mathrm{mg}, 2 \mathrm{mmol})$ in DCM $(30 \mathrm{~mL})$ was added $12 \mathrm{~mL}$ solution of $\mathrm{Na}_{2} \mathrm{CO}_{3}(25 \%)$ followed by $3.4 \mathrm{mmol}$ $(587 \mathrm{mg})$ of $m$-CPBA dropwise at $0{ }^{\circ} \mathrm{C}$. The mixture was stirred at the same temperature and monitored with TLC until the reactant was consumed completely. At the end of the epoxidation process, the mixture was extracted with DCM, dried over anhydrous $\mathrm{MgSO}_{4}$, filtered, and concentrated under reduced pressure. The crude mixture was chromatographed on $\mathrm{NEt}_{3}$-pretreated short silica gel column which afforded the enyne oxirane $(Z)-\mathbf{1 i}$ or $(Z)-1 \mathbf{i}^{\prime}$ in about $85 \%$ purities as pale yellow oil products (hexane-EtAc, yield of $(Z)-1 \mathbf{i}: 300 \mathrm{mg}$, $57 \%$; yield of (Z)-1i': $158 \mathrm{mg}, 30 \%)$.

(Z)-1i: ${ }^{1} \mathrm{H}$ NMR $\left(400 \mathrm{MHz}, \mathrm{C}_{6} \mathrm{D}_{6}\right) \delta: 5.32(\mathrm{~d}, J=8.7 \mathrm{~Hz}, 1 \mathrm{H})$, $3.90(\mathrm{dd}, J=8.7,2.3 \mathrm{~Hz}, 1 \mathrm{H}), 2.71(\mathrm{td}, J=5.5,2.3 \mathrm{~Hz}, 1 \mathrm{H}), 2.11(\mathrm{t}$, $J=6.8 \mathrm{~Hz}, 2 \mathrm{H}), 2.10(\mathrm{t}, J=6.4 \mathrm{~Hz}, 2 \mathrm{H}), 1.54-1.15(\mathrm{~m}, 14 \mathrm{H}), 0.84-$ $0.70(\mathrm{~m}, 9 \mathrm{H}) ;{ }^{13} \mathrm{C}\{1 \mathrm{H}\} \mathrm{NMR}\left(100 \mathrm{MHz}, \mathrm{C}_{6} \mathrm{D}_{6}\right) \delta: 137.5,133.3,95.7$, 78.6, 59.6, 56.4, 37.4, 31.8, 30.7, 30.3, 28.0, 22.4, 22.0, 21.8, 19.0, 13.7, 13.6, 13.3 .

(Z)-1i': ${ }^{1} \mathrm{H}$ NMR $\left(400 \mathrm{MHz}, \mathrm{CDCl}_{3}\right) \delta: 5.38(\mathrm{~d}, J=9.0,1 \mathrm{H}), 3.90$ $(\mathrm{dd}, J=9.0,4.8 \mathrm{~Hz}, 1 \mathrm{H}), 3.11(\mathrm{q}, J=4.8 \mathrm{~Hz}, 1 \mathrm{H}), 2.37(\mathrm{t}, J=6.8 \mathrm{~Hz}$, 

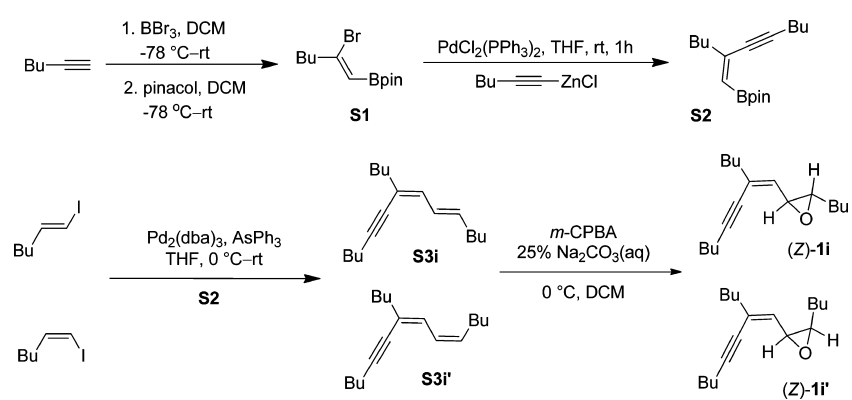

$2 \mathrm{H}), 2.15(\mathrm{td}, J=7.6,0.8 \mathrm{~Hz}, 2 \mathrm{H}), 1.60-1.25(\mathrm{~m}, 14 \mathrm{H}), 0.95-0.88$ $(\mathrm{m}, 9 \mathrm{H}) ;{ }^{13} \mathrm{C}\{1 \mathrm{H}\} \mathrm{NMR}\left(100 \mathrm{MHz}, \mathrm{CDCl}_{3}\right) \delta: 130.8,128.9,96.4,78.1$, 59.1, 55.4, 37.5, 30.8, 30.2, 28.5, 28.2, 22.5, 21.9, 21.9, 19.2, 14.0, 13.9, 13.8 .

Synthesis of (Z)-1a. A hexane solution of BuLi $(4.8 \mathrm{~mL}, 12 \mathrm{mmol}$, $2.5 \mathrm{M})$ was added dropwise to a solution of isopropyl(triphenyl)phosphonium iodide $(4.32 \mathrm{~g}, 10 \mathrm{mmol})$ in THF $(30 \mathrm{~mL})$ at $0{ }^{\circ} \mathrm{C}$ and stirred for further $1 \mathrm{~h}$. The enyne aldehyde $(Z)-S 4 a(1.8 \mathrm{~g}, 12 \mathrm{mmol})$, which was synthesized as prescribed elsewhere, ${ }^{4 a, b}$ was added dropwise to the resulting mixture and stirred for $1 \mathrm{~h}$ at $\mathrm{rt}$. The reaction was quenched by the addition of saturated $\mathrm{NH}_{4} \mathrm{Cl}$ (aq) solution, and the organic layer was extracted with diethyl ether. The combined extracts were dried over $\mathrm{MgSO}_{4}$ and chromatographed on a silica gel column to obtain pure S3a (hexane/EtOAc, yield: $1.65 \mathrm{~g}, 78 \%){ }^{19}$

The epoxidation of S3a $(352 \mathrm{mg}, 2 \mathrm{mmol})$ and isolation of the product $(Z)-\mathbf{1}$ a was performed as specified for $(Z)-\mathbf{1 i}$ (hexane-EtOAc, yield: $0.27 \mathrm{~g}, 70 \%)$.

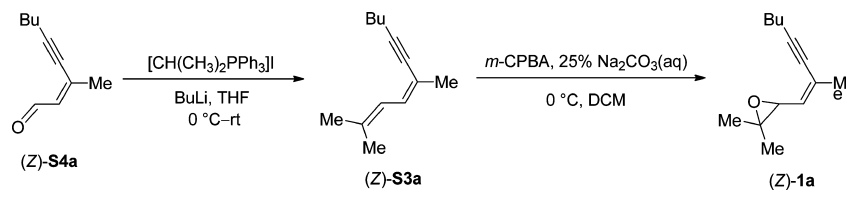

(Z)-1a: ${ }^{1} \mathrm{H}$ NMR $\left(400 \mathrm{MHz}, \mathrm{CDCl}_{3}\right) \delta: 5.38(\mathrm{dq}, J=8.9,1.5 \mathrm{~Hz}$ $1 \mathrm{H}), 3.65(\mathrm{~d}, J=8.9 \mathrm{~Hz}, 1 \mathrm{H}), 2.34(\mathrm{t}, J=6.8 \mathrm{~Hz}, 2 \mathrm{H}), 1.87(\mathrm{~s}, 3 \mathrm{H})$, $1.56-1.36(\mathrm{~m}, 4 \mathrm{H}), 1.35(\mathrm{~s}, 3 \mathrm{H}), 1.27(\mathrm{~s}, 3 \mathrm{H}), 0.9(\mathrm{t}, J=7.2 \mathrm{~Hz}, 3 \mathrm{H})$; ${ }^{13} \mathrm{C}\{1 \mathrm{H}\}$ NMR $\left(100 \mathrm{MHz}, \mathrm{CDCl}_{3}\right) \delta: 130.7,125.5,95.9,79.1,62.6$, 60.5, 30.9, 24.9, 24.2, 22.1, 19.6, 19.3, 13.7 .

Syntheses of (Z)-1b-e,h,k-s. A general procedure for the syntheses of noncyclic enyne aldehydes (S4) used here was reported elsewhere. ${ }^{4 a, b}$

To a solution of $\mathrm{NaH}$ (1.1 equiv) in THF $(2.5 \mathrm{~mL} / \mathrm{mmol} \mathrm{S4})$ was added triethyl phosphonoacetate ( 1.2 equiv) at $0{ }^{\circ} \mathrm{C}$, and the mixture stirred for $1 \mathrm{~h}$ at room temperature. Subsequently, to the reaction mixture was added $\$ 4(6.5-10 \mathrm{mmol})$ dropwise at $-78^{\circ} \mathrm{C}$ and stirred for $1 \mathrm{~h}$ at room temperature. The reaction was terminated by the addition of saturated $\mathrm{NH}_{4} \mathrm{Cl}$ and extracted with $\mathrm{Et}_{2} \mathrm{O}$. The organic layer was dried over $\mathrm{MgSO}_{4}$, filtered, and concentrated under reduced pressure to obtain $S 5$ with $E / Z$ isomeric ratios varying in the range of 97:3 to $95: 5 .^{20}$ The crude mixture was purified on silica gel column (hexane-EtAc) to obtain $\mathbf{S 5}$ in pure isomeric form (yields of $(2 E, 4 Z)$ S5: $\mathrm{R}^{1}=\mathrm{Bu}, \mathrm{R}^{2}=\mathrm{Me}, 3.17 \mathrm{~g}, 72 \% ; \mathrm{R}^{1}=\mathrm{H}, \mathrm{R}^{2}=\mathrm{Me}, 2.30 \mathrm{~g}, 70 \% ; \mathrm{R}^{1}=$ $\mathrm{Bu}, \mathrm{R}^{2}=\mathrm{H}, 1.48 \mathrm{~g}, 72 \% ; \mathrm{R}^{1}=\mathrm{Bu}, \mathrm{R}^{2}=\mathrm{Cy}, 1.4 \mathrm{~g}, 68 \% ; \mathrm{R}^{1}=\mathrm{Cy}, \mathrm{R}^{2}=$ $\mathrm{Me}, 1.97 \mathrm{~g}, 80 \%$; $\mathrm{R}^{1}=t-\mathrm{Bu}, \mathrm{R}^{2}=\mathrm{Me}, 1.80 \mathrm{~g}, 82 \% ; \mathrm{R}^{1}=\mathrm{Bu}, \mathrm{R}^{2}=t-\mathrm{Bu}$, $\left.2.12 \mathrm{~g}, 81 \% ; \mathrm{R}^{1}=\mathrm{Ph}, \mathrm{R}^{2}=\mathrm{Me}, 2.04 \mathrm{~g}, 85 \%\right)$.

A DIBALH ( $\sim 3$ eq, 1.0 $\mathrm{M}$ in cyclohexane) solution was added dropwise to the solution of S5 in DCM $(\sim 6 \mathrm{~mL} / \mathrm{mmol} \mathrm{S5})$ at $-78^{\circ} \mathrm{C}$. After the reaction mixture was stirred for $4 \mathrm{~h}$ at the same temperature, $1 \mathrm{M} \mathrm{HCl}(\mathrm{aq})$ solution was added before extracting with DCM. The organic layers were combined, washed with brine, dried over $\mathrm{MgSO}_{4}$, filtered, and concentrated under reduced pressure. The crude mixture was subjected to silica gel column chromatography to purify the corresponding S6 compound (hexane-EtOAc; yields of (2E,4Z)-S6: $\mathrm{R}^{1}=\mathrm{Bu}, \mathrm{R}^{2}=\mathrm{Me}, 2.18 \mathrm{~g}, 85 \% ; \mathrm{R}^{1}=\mathrm{H}, \mathrm{R}^{2}=\mathrm{Me}, 1.45 \mathrm{~g}, 85 \% ; \mathrm{R}^{1}=\mathrm{Bu}$, $\mathrm{R}^{2}=\mathrm{H}, 1.12 \mathrm{~g}, 95 \% ; \mathrm{R}^{1}=\mathrm{Bu}, \mathrm{R}^{2}=\mathrm{Cy}, 1.1 \mathrm{~g}, 94 \% ; \mathrm{R}^{1}=\mathrm{Cy}, \mathrm{R}^{2}=\mathrm{Me}$, $1.55 \mathrm{~g}, 95 \% ; \mathrm{R}^{1}=t-\mathrm{Bu}, \mathrm{R}^{2}=\mathrm{Me}, 1.39 \mathrm{~g}, 95 \% ; \mathrm{R}^{1}=\mathrm{Bu}, \mathrm{R}^{2}=t$-Bu, $1.6 \mathrm{~g}$, $\left.90 \% ; \mathrm{R}^{1}=\mathrm{Ph}, \mathrm{R}^{2}=\mathrm{Me}, 1.6 \mathrm{~g}, 92 \%\right)$.

The epoxidation of $\mathbf{S 6}(2 \mathrm{mmol})$ and isolation of the corresponding S7 products were performed as specified for $(Z)-\mathbf{1 i}$ (yields of $(Z)-\mathbf{S 7}$ :
$\mathrm{R}^{1}=\mathrm{Bu}, \mathrm{R}^{2}=\mathrm{Me},(Z)-1 \mathrm{~d}, 0.23 \mathrm{~g}, 60 \% ; \mathrm{R}^{1}=\mathrm{H}, \mathrm{R}^{2}=\mathrm{Me}, 0.17 \mathrm{~g}, 60 \%$; $\mathrm{R}^{1}=\mathrm{Bu}, \mathrm{R}^{2}=\mathrm{H}, 0.19 \mathrm{~g}, 55 \% ; \mathrm{R}^{1}=\mathrm{Bu}, \mathrm{R}^{2}=\mathrm{Cy}, 0.26 \mathrm{~g}, 49 \% ; \mathrm{R}^{1}=\mathrm{Cy}$, $\mathrm{R}^{2}=\mathrm{Me}, 0.25 \mathrm{~g}, 57 \% ; \mathrm{R}^{1}=t-\mathrm{Bu}, \mathrm{R}^{2}=\mathrm{Me},(Z)-1 \mathrm{o}, 0.25 \mathrm{~g}, 65 \% ; \mathrm{R}^{1}=$ $\mathrm{Bu}, \mathrm{R}^{2}=t$-Bu, $0.24 \mathrm{~g}, 50 \% ; \mathrm{R}^{1}=\mathrm{Ph}, \mathrm{R}^{2}=\mathrm{Me}, 0.26 \mathrm{~g}, 60 \%$ ).

As for the alkylation of the pendant hydroxyl group of S7, a suspension of sodium hydride (1.1 equiv) in DMF $(1 \mathrm{~mL})$ was added to a solution of $\mathbf{S 7}(1 \mathrm{mmol})$ in DMF $(1 \mathrm{~mL} / \mathrm{mmol} \mathrm{S7})$ at $-20{ }^{\circ} \mathrm{C}$. The mixture was stirred for further $30 \mathrm{~min}$ before the addition of methyl iodide ( 1.2 equiv) or benzyl bromide (1.2 equiv). The mixture was stirred for $4 \mathrm{~h}$ at the same temperature, and then the reaction was terminated by the addition of $\mathrm{MeOH}(5 \mathrm{~mL})$ and brine $(5 \mathrm{~mL})$ and extracted with DCM. The combined extracts were dried over $\mathrm{MgSO}_{4}$, filtered, and concentrated under reduced pressure. The crude mixture was subjected to column chromatography over $\mathrm{NEt}_{3}$-pretreated short silica gel column to afford the corresponding alkoxy-substituted enyne oxirane products as colorless oil (hexane-EtOAc, yields $(Z)-\mathbf{1 b}$, 0.18 g, 87\%; (Z)-1c, 0.22 g, 77\%; (Z)-1h, 0.17 g, 90\%; (Z)-1k, 0.24 g, 87\%; (Z)-11, 0.21 g, 83\%; (Z)-1m, 0.13 g, 87\%; (Z)-1n, 0.19 g; 84\%; (Z)-1o, 0.19 g, 90\%; (Z)-1p, 0.24 g, 85\%; (Z)-1s, 0.20 g, 87\%). ${ }^{22}$

A mixture of $(Z)-1 d(194, \mathrm{mg}, 1 \mathrm{mmol})$ or $(Z)-1 \mathrm{q}(227 \mathrm{mg}$, $1.17 \mathrm{mmol}), \mathrm{Et}_{3} \mathrm{~N}(0.18 \mathrm{~mL}, 1.3 \mathrm{mmol}), t$-butyldimethylsilyl chloride $(0.2 \mathrm{~g}, 1.3 \mathrm{mmol})$, and 4-dimethylaminopyridine (DMAP) (15 mg, $0.12 \mathrm{mmol})$ in $\mathrm{CH}_{2} \mathrm{Cl}_{2}(12 \mathrm{~mL})$ was stirred at room temperature for $24 \mathrm{~h}$. Then, the reaction was terminated by water, and the content of the reaction flask was extracted with DCM. The organic solution was dried with $\mathrm{MgSO}_{4}$, filtered, and evaporated. The residue was chromatographed over $\mathrm{NEt}_{3}$-pretreated short silica gel column to afford silylated enyne oxiranes as a colorless oil (hexane-EtOAc, yields (Z)-1e, $0.28 \mathrm{~g}$, 79\%; (Z)-1r, $0.25 \mathrm{~g}, 81 \%){ }^{23}$

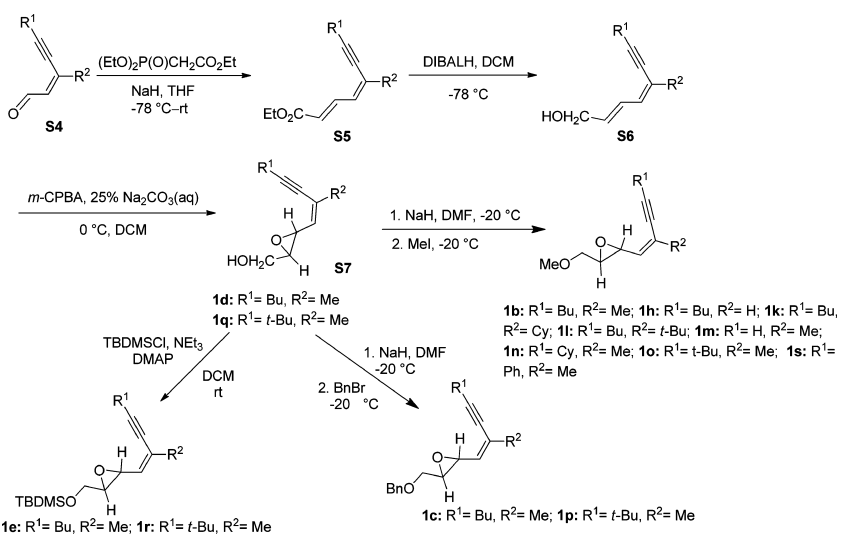

Synthesis of (E)-1b. Synthesis of (E)-1b was performed starting from $(E)$-configured $\mathbf{S} 4$ following the same method employed for the synthesis of $(Z)-\mathbf{1 b}$. Yields: $\left(\mathrm{R}^{1}=\mathrm{Bu}, \mathrm{R}^{2}=\mathrm{Me}\right):(2 E, 4 E)-\mathbf{S} 5 \mathbf{b}, 1.12 \mathrm{~g}$, 79\%; (2E,4E)-S6b, $0.83 \mathrm{~g}, 91 \%$; (E)-S7b (produced from $4.6 \mathrm{mmol}$ of (2E,4E)-S6b), $0.43 \mathrm{~g}$, 47\%; (E)-1b (produced from $2.2 \mathrm{mmol}$ of (E)-S7b), $0.40 \mathrm{~g}, 88 \%$.

(Z)-1b: ${ }^{1} \mathrm{H}$ NMR $\left(400 \mathrm{MHz}, \mathrm{CDCl}_{3}\right) \delta: 5.23(\mathrm{dd}, J=8.9,1.2 \mathrm{~Hz}$, $1 \mathrm{H}), 3.74(\mathrm{dd}, J=8.9,2.4 \mathrm{~Hz}, 1 \mathrm{H}), 3.71(\mathrm{dd}, J=11.6,3.2 \mathrm{~Hz}, 1 \mathrm{H})$, $3.40(\mathrm{dd}, J=11.6,5.7 \mathrm{~Hz}, 1 \mathrm{H}), 3.40(\mathrm{~s}, 3 \mathrm{H}), 3.08(\mathrm{ddd}, J=5.7,3.2$, $2.4 \mathrm{~Hz}, 1 \mathrm{H}), 2.35(\mathrm{t}, J=7.2 \mathrm{~Hz}, 2 \mathrm{H}), 1.87(\mathrm{~d}, J=1.2 \mathrm{~Hz}, 3 \mathrm{H}), 1.55-$ $1.40(\mathrm{~m}, 4 \mathrm{H}), 0.92(\mathrm{t}, J=7.2 \mathrm{~Hz}, 3 \mathrm{H}) ;{ }^{13} \mathrm{C}\{1 \mathrm{H}\} \mathrm{NMR}(100 \mathrm{MHz}$, $\left.\mathrm{CDCl}_{3}\right) \delta: 131.5,125.7,95.9,78.7,72.5,59.2,58.3,54.2,30.7,23.8$, 21.9, 19.1, 13.6.

(E)-1b: $\left(400 \mathrm{MHz}, \mathrm{CDCl}_{3}\right) \delta: 5.35(\mathrm{dd}, J=9.0,1.0 \mathrm{~Hz}, 1 \mathrm{H}), 3.67$ $(\mathrm{dd}, J=11.3,3.0 \mathrm{~Hz}, 1 \mathrm{H}), 3.48(\mathrm{dd}, J=9.0,2.2 \mathrm{~Hz}, 1 \mathrm{H}), 3.44(\mathrm{dd}, J=$ $11.3,5.1 \mathrm{~Hz}, 1 \mathrm{H}), 3.38(\mathrm{~s}, 3 \mathrm{H}), 3.06(\mathrm{ddd}, J=5.1,3.0,2.2 \mathrm{~Hz}, 1 \mathrm{H})$, $2.28(\mathrm{t}, J=6.8 \mathrm{~Hz}, 2 \mathrm{H}), 1.93(\mathrm{~d}, J=1.0 \mathrm{~Hz}, 3 \mathrm{H}), 1.54-1.37(\mathrm{~m}, 4 \mathrm{H})$, $0.90(\mathrm{t}, J=7.6 \mathrm{~Hz}, 3 \mathrm{H}) ;{ }^{13} \mathrm{C}\{1 \mathrm{H}\} \mathrm{NMR}\left(100 \mathrm{MHz}, \mathrm{CDCl}_{3}\right) \delta: 131.3$, 125.2, 90.3, 82.4, 80.0, 59.2, 58.6, 51.9, 30.7, 21.9, 18.9, 18.2, 13.6.

(Z)-1c: ${ }^{1} \mathrm{H}$ NMR $\left(400 \mathrm{MHz}, \mathrm{C}_{6} \mathrm{D}_{6}\right) \delta: 7.24-7.22(\mathrm{~m}, 2 \mathrm{H}), 7.14-$ $7.10(\mathrm{~m}, 2 \mathrm{H}), 7.05(\mathrm{dt}, J=7.2,1.6 \mathrm{~Hz}, 1 \mathrm{H}), 5.14(\mathrm{dd}, J=8.9,1.6 \mathrm{~Hz}$, $1 \mathrm{H}), 4.35\left(\mathrm{~d}, \mathrm{~A}\right.$ of $\left.\mathrm{AB}, J_{\mathrm{AB}}=12.1 \mathrm{~Hz}, 1 \mathrm{H}\right), 4.31\left(\mathrm{~d}, \mathrm{~B}\right.$ of $\mathrm{AB}, J_{\mathrm{AB}}=12.1$ $\mathrm{Hz}, 1 \mathrm{H}), 4.01(\mathrm{dd}, J=8.9,2.2 \mathrm{~Hz}, 1 \mathrm{H}), 3.45(\mathrm{dd}, J=11.4,3.0 \mathrm{~Hz}$, $1 \mathrm{H}), 3.25(\mathrm{dd}, J=11.4,5.5 \mathrm{~Hz}, 1 \mathrm{H}), 2.95$ (ddd, $J=5.5,3.0,2.2 \mathrm{~Hz}$, 
$1 \mathrm{H}), 2.06(\mathrm{t}, J=6.8 \mathrm{~Hz}, 2 \mathrm{H}), 1.71(\mathrm{~d}, J=1.6 \mathrm{~Hz}, 3 \mathrm{H}), 1.29-1.16(\mathrm{~m}$, $4 \mathrm{H}), 0.7(\mathrm{t}, J=7.2 \mathrm{~Hz}, 3 \mathrm{H}) ;{ }^{13} \mathrm{C}\{1 \mathrm{H}\}$ NMR $\left(100 \mathrm{MHz}, \mathrm{C}_{6} \mathrm{D}_{6}\right) \delta$ : $138.5,132.8,128.2,127.4,127.3,124.8,95.5,79.1,72.8,70.0,58.2$, 53.8, 30.6, 23.5, 21.8, 18.9, 13.3 .

(Z)-1d: ${ }^{1} \mathrm{H}$ NMR $\left(400 \mathrm{MHz}, \mathrm{CDCl}_{3}\right) \delta: 5.25(\mathrm{dd}, J=9.0,1.4 \mathrm{~Hz}$, $1 \mathrm{H}), 3.96(\mathrm{dd}, J=12.6,2.3 \mathrm{~Hz}, 1 \mathrm{H}), 3.87(\mathrm{dd}, J=9.0,2.3 \mathrm{~Hz}, 1 \mathrm{H})$, $3.68(\mathrm{dd}, J=12.6,4.1 \mathrm{~Hz}, 1 \mathrm{H}), 3.11-3.09(\mathrm{~m}, 1 \mathrm{H}), 2.36(\mathrm{t}, J=7.0 \mathrm{~Hz}$, $2 \mathrm{H}), 1.88(\mathrm{~d}, J=1.4 \mathrm{~Hz}, 3 \mathrm{H}), 1.56-1.39(\mathrm{~m}, 4 \mathrm{H}), 0.92(\mathrm{t}, J=7.2 \mathrm{~Hz}$ $3 \mathrm{H}) ;{ }^{13} \mathrm{C}\{1 \mathrm{H}\}$ NMR $\left(100 \mathrm{MHz}, \mathrm{C}_{6} \mathrm{D}_{6}\right) \delta: 132.5,124.8,95.7,79.1$, 61.1, 59.5, 53.7, 30.6, 23.5, 21.8, 19.0, 13.3 .

(Z)-1e: ${ }^{1} \mathrm{H}$ NMR $\left(400 \mathrm{MHz}, \mathrm{CDCl}_{3}\right) \delta: 5.22(\mathrm{dd}, J=9.1,1.5 \mathrm{~Hz}$, $1 \mathrm{H}), 3.86(\mathrm{dd}, J=12.1,3.2 \mathrm{~Hz}, 1 \mathrm{H}), 3.76(\mathrm{dd}, J=9.1,2.4 \mathrm{~Hz}, 1 \mathrm{H})$, $3.71(\mathrm{dd}, J=12.1,4.8 \mathrm{~Hz}, 1 \mathrm{H}), 3.00(\mathrm{ddd}, J=4.8,3.2,2.4 \mathrm{~Hz}, 1 \mathrm{H})$, $2.33(\mathrm{t}, J=7.2 \mathrm{~Hz}, 2 \mathrm{H}), 1.86(\mathrm{~d}, J=1.5 \mathrm{~Hz}, 3 \mathrm{H}), 1.56-1.37(\mathrm{~m}, 4 \mathrm{H})$, $0.91(\mathrm{t}, J=6.8 \mathrm{~Hz}, 3 \mathrm{H}), 0.89(\mathrm{~s}, 9 \mathrm{H}), 0.07(\mathrm{~s}, 3 \mathrm{H}), 0.06(\mathrm{~s}, 3 \mathrm{H})$. ${ }^{13} \mathrm{C}\{1 \mathrm{H}\}$ NMR $\left(100 \mathrm{MHz}, \mathrm{CDCl}_{3}\right) \delta: 131.9,125.3,95.7,78.7,63.0$, 60.1, 54.3, 30.8, 25.8, 23.8, 22.0, 19.1, 18.3, 13.6, -5.3, -5.4.

(Z)-1h: ${ }^{1} \mathrm{H}$ NMR $\left(400 \mathrm{MHz}, \mathrm{CDCl}_{3}\right) \delta: 5.75$ (dtd, $J=10.8,2.4,0.8$ $\mathrm{Hz}, 1 \mathrm{H}), 5.44(\mathrm{dd}, J=10.8,8.8 \mathrm{~Hz}, 1 \mathrm{H}), 3.80(\mathrm{dd}, J=8.8,2.2 \mathrm{~Hz}$, $1 \mathrm{H}), 3.74(\mathrm{dd}, J=11.2,2.8 \mathrm{~Hz}, 1 \mathrm{H}), 3.44-3.38(\mathrm{~m}, 1 \mathrm{H}), 3.41(\mathrm{~s}, 3 \mathrm{H})$, $3.11(\mathrm{dt}, J=5.4,2.6 \mathrm{~Hz}, 1 \mathrm{H}), 2.36(\mathrm{td}, J=6.8,2.3 \mathrm{~Hz}, 2 \mathrm{H}), 1.58-1.41$ $(\mathrm{m}, 4 \mathrm{H}), 0.92(\mathrm{t}, J=7.2 \mathrm{~Hz}, 3 \mathrm{H}) ;{ }^{13} \mathrm{C}\{1 \mathrm{H}\} \operatorname{NMR}\left(100 \mathrm{MHz}, \mathrm{CDCl}_{3}\right)$ $\delta: 137.2,115.4,97.1,72.3,59.2,58.2,53.3,50.4,30.6,21.9,19.2$, 13.5.

(Z)-1k: ${ }^{1} \mathrm{H}$ NMR (400 MHz, $\left.\mathrm{CDCl}_{3}\right) \delta: 5.21(\mathrm{~d}, J=8.7 \mathrm{~Hz}, 1 \mathrm{H})$, 3.77 (dd, $J=8.7,2.4 \mathrm{~Hz}, 1 \mathrm{H}), 3.72(\mathrm{dd}, J=11.5,3.1 \mathrm{~Hz}, 1 \mathrm{H}), 3.38$ $(\mathrm{dd}, J=11.5,5.7 \mathrm{~Hz}, 1 \mathrm{H}), 3.40(\mathrm{~s}, 3 \mathrm{H}), 3.07(\mathrm{dt}, J=5.7,2.4 \mathrm{~Hz}, 1 \mathrm{H})$, $2.37(\mathrm{t}, J=7.2 \mathrm{~Hz}, 2 \mathrm{H}), 2.02-1.97(\mathrm{~m}, 1 \mathrm{H}), 1.76-1.20(\mathrm{~m}, 14 \mathrm{H})$, $0.92(\mathrm{t}, J=7.2 \mathrm{~Hz}, 3 \mathrm{H}) ;{ }^{13} \mathrm{C}\{1 \mathrm{H}\} \operatorname{NMR}\left(100 \mathrm{MHz} \mathrm{CDCl}_{3}\right) \delta: 136.3$, 129.1, 97.0, 76.7, 72.6, 59.2, 58.5, 54.3, 45.4, 31.7, 31.6, 30.8, 29.7, 26.2, 26.0, 22.0, 19.2, 13,6.

(Z)-11: ${ }^{1} \mathrm{H}$ NMR (400 MHz, $\left.\mathrm{CDCl}_{3}\right) \delta: 5.24(\mathrm{~d}, J=8.9 \mathrm{~Hz}, 1 \mathrm{H})$, $3.81(\mathrm{dd}, J=8.9,2.4 \mathrm{~Hz}, 1 \mathrm{H}), 3.74(\mathrm{dd}, J=11.2,3.1 \mathrm{~Hz}, 1 \mathrm{H}), 3.42-$ $3.38(\mathrm{~m}, 1 \mathrm{H}), 3.41(\mathrm{~s}, 3 \mathrm{H}), 3.10(\mathrm{ddd}, J=5.6,3.1,2.4 \mathrm{~Hz}, 1 \mathrm{H}), 2.39$ $(\mathrm{t}, J=7.2 \mathrm{~Hz}, 2 \mathrm{H}), 1.59-1.41(\mathrm{~m}, 4 \mathrm{H}), 1.11(\mathrm{~s}, 9 \mathrm{H}), 0.93(\mathrm{t}, J=7.6$ $\mathrm{Hz}, 3 \mathrm{H}) ;{ }^{13} \mathrm{C}\{1 \mathrm{H}\}$ NMR $\left(100 \mathrm{MHz}, \mathrm{CDCl}_{3}\right) \delta: 140.2,127.4,97.4$, 72.7, 59.2, 58.5, 54.7, 36.1, 30.8, 28.9, 21.9, 19.2, 13.6.

(Z)-1m: ${ }^{1} \mathrm{H}$ NMR $\left(400 \mathrm{MHz}, \mathrm{C}_{6} \mathrm{D}_{6}\right) \delta: 5.10(\mathrm{dq}, J=8.9,1.0 \mathrm{~Hz}$, $1 \mathrm{H}), 3.84(\mathrm{dd}, J=8.9,2.0 \mathrm{~Hz}, 1 \mathrm{H}), 3.25(\mathrm{dd}, J=11.5,3.0 \mathrm{~Hz}, 1 \mathrm{H})$, $3.06-2.99(\mathrm{~m}, 1 \mathrm{H}), 3.05(\mathrm{~s}, 3 \mathrm{H}), 2.83(\mathrm{ddd}, J=5.5,3.0,2.0 \mathrm{~Hz}, 1 \mathrm{H})$, $2.74(\mathrm{~s}, 1 \mathrm{H}), 1.57(\mathrm{~d}, J=1.0 \mathrm{~Hz}, 3 \mathrm{H}) ;{ }^{13} \mathrm{C}\{1 \mathrm{H}\}$ NMR $(100 \mathrm{MHz}$, $\left.\mathrm{C}_{6} \mathrm{D}_{6}\right) \delta: 136.0,122.9,82.4,81.5,72.2,58.4,58.0,53.2,22.7$.

(Z)-1n: ${ }^{1} \mathrm{H}$ NMR (400 MHz, $\left.\mathrm{C}_{6} \mathrm{D}_{6}\right) \delta: 5.14(\mathrm{dq}, J=9.0,1.4 \mathrm{~Hz}$, $1 \mathrm{H}), 4.01(\mathrm{dd}, J=9.0,2.0 \mathrm{~Hz}, 1 \mathrm{H}), 3.35(\mathrm{dd}, J=11.3,3.2 \mathrm{~Hz}, 1 \mathrm{H})$, $3.16(\mathrm{dd}, J=11.3,5.5 \mathrm{~Hz}, 1 \mathrm{H}), 3.09(\mathrm{~s}, 3 \mathrm{H}), 2.91$ (ddd, $J=5.5,3.2$, $2.0 \mathrm{~Hz}, 1 \mathrm{H}), 2.34(\mathrm{~m}, 1 \mathrm{H}), 1.71(\mathrm{~d}, J=1.4 \mathrm{~Hz}, 3 \mathrm{H}), 1.66-1.03(\mathrm{~m}$, $10 \mathrm{H}) ;{ }^{13} \mathrm{C}\{1 \mathrm{H}\}$ NMR $\left(100 \mathrm{MHz}, \mathrm{C}_{6} \mathrm{D}_{6}\right) \delta: 132.7,124.7,99.5,79.2$, 72.4, 58.5, 58.1, 53.7, 32.5, 32.4, 29.6, 25.7, 24.6, 23.5.

(Z)-10: ${ }^{1} \mathrm{H}$ NMR (400 MHz, $\left.\mathrm{C}_{6} \mathrm{D}_{6}\right) \delta: 5.12(\mathrm{dd}, J=8.5,1.3 \mathrm{~Hz}$, $1 \mathrm{H}), 3.95(\mathrm{dd}, J=8.5,2.3 \mathrm{~Hz}, 1 \mathrm{H}), 3.34(\mathrm{dd}, J=11.5,3.1 \mathrm{~Hz}, 1 \mathrm{H})$, $3.16(\mathrm{dd}, J=11.5,5.5 \mathrm{~Hz}, 1 \mathrm{H}), 3.09(\mathrm{~s}, 3 \mathrm{H}), 2.90$ (ddd, $J=5.5,3.1$, $2.3 \mathrm{~Hz}, 1 \mathrm{H}), 1.68(\mathrm{~d}, J=1.3 \mathrm{~Hz}, 3 \mathrm{H}), 1.12(\mathrm{~s}, 9 \mathrm{H}) ;{ }^{13} \mathrm{C}\{1 \mathrm{H}\} \mathrm{NMR}$ $\left(100 \mathrm{MHz}, \mathrm{C}_{6} \mathrm{D}_{6}\right) \delta: 132.8,124.5,103.4,77.7,72.4,58.5,58.0,53.7$, $30.6,27.9,23.4$

(Z)-1p: ${ }^{1} \mathrm{H}$ NMR $\left(400 \mathrm{MHz}, \mathrm{CDCl}_{3}\right) \delta: 7.35-7.28(\mathrm{~m}, 5 \mathrm{H}), 5.22$ $(\mathrm{dq}, J=8.9,1.4 \mathrm{~Hz}, 1 \mathrm{H}), 4.61\left(\mathrm{~d}, \mathrm{~A}\right.$ of $\left.\mathrm{AB}, J_{\mathrm{AB}}=12.0 \mathrm{~Hz}, 1 \mathrm{H}\right), 4.57$ $\left(\mathrm{d}, \mathrm{B}\right.$ of $\left.\mathrm{AB}, J_{\mathrm{AB}}=12.0 \mathrm{~Hz}, 1 \mathrm{H}\right), 3.81(\mathrm{dd}, J=11.3,3.0 \mathrm{~Hz}, 1 \mathrm{H}), 3.76$ (dd, $J=8.9,2.2 \mathrm{~Hz}, 1 \mathrm{H}), 3.53(\mathrm{dd}, J=11.3,5.5 \mathrm{~Hz}, 1 \mathrm{H}), 3.13$ (ddd, $J=5.5,3.0,2.2 \mathrm{~Hz}, 1 \mathrm{H}), 1.86(\mathrm{~d}, J=1.4 \mathrm{~Hz}, 3 \mathrm{H}), 1.24(\mathrm{~s}, 9 \mathrm{H})$; ${ }^{13} \mathrm{C}\{1 \mathrm{H}\}$ NMR $\left(100 \mathrm{MHz}, \mathrm{CDCl}_{3}\right) \delta: 140.0,137.9,131.3,128.4$, 127.7, 125.7, 104.0, 77.1, 73.3, 70.1, 58.6, 54.3, 31.0, 28.1, 23.8.

(Z)-1q: ${ }^{1} \mathrm{H}$ NMR $\left(400 \mathrm{MHz}, \mathrm{C}_{6} \mathrm{D}_{6}\right) \delta: 5.17(\mathrm{dd}, J=8.9,1.1 \mathrm{~Hz}$, $1 \mathrm{H}), 4.1(\mathrm{dd}, J=8.9,2.3 \mathrm{~Hz}, 1 \mathrm{H}), 3.54-3.48(\mathrm{~m}, 1 \mathrm{H}), 3.34-3.30(\mathrm{~m}$, $1 \mathrm{H}), 2.75-2.73(\mathrm{~m}, 1 \mathrm{H}), 1.73(\mathrm{~d}, J=1.1 \mathrm{~Hz}, 3 \mathrm{H}), 1.17(\mathrm{~s}, 9 \mathrm{H})$; ${ }^{13} \mathrm{C}\{1 \mathrm{H}\}$ NMR $\left(100 \mathrm{MHz}, \mathrm{C}_{6} \mathrm{D}_{6}\right) \delta: 132.5,124.6,103.7,77.6,61.0$, 59.4, 53.6, 30.6, 27.9, 23.4 .

(Z)-1r: ${ }^{1} \mathrm{H}$ NMR $\left(400 \mathrm{MHz}, \mathrm{CDCl}_{3}\right) \delta: 5.21(\mathrm{~d}, J=9.0 \mathrm{~Hz}, 1 \mathrm{H})$, $3.87(\mathrm{dd}, J=12.0,3.2 \mathrm{~Hz}, 1 \mathrm{H}), 3.74(\mathrm{~m}, 2 \mathrm{H}), 3.01(\mathrm{~m}, 1 \mathrm{H}), 1.86(\mathrm{~s}$, $3 \mathrm{H}), 1.26(\mathrm{~s}, 9 \mathrm{H}), 0.90(\mathrm{~s}, 9 \mathrm{H}), 0.08(\mathrm{~s}, 6 \mathrm{H}) ;{ }^{13} \mathrm{C}\{1 \mathrm{H}\}$ NMR: $(100$ $\left.\mathrm{MHz}, \mathrm{CDCl}_{3}\right) \delta: 131.6,125.3,103.8,77.1,62.9,60.2,54.3,31.0,28.1$, $25.9,23.9,18.3,-5,3$.
(Z)-1s: ${ }^{1} \mathrm{H}$ NMR $\left(400 \mathrm{MHz}, \mathrm{CDCl}_{3}\right) \delta: 7.46-7.44(\mathrm{~m}, 2 \mathrm{H}), 7.34-$ $7.32(\mathrm{~m}, 3 \mathrm{H}), 5.38(\mathrm{dq}, J=9.0,1.5 \mathrm{~Hz}, 1 \mathrm{H}), 3.84(\mathrm{dd}, J=9.0,2.4 \mathrm{~Hz}$, $1 \mathrm{H}), 3.74(\mathrm{dd}, J=11.7,3.2 \mathrm{~Hz}, 1 \mathrm{H}), 3.43(\mathrm{dd}, J=11.7,6.0 \mathrm{~Hz}, 1 \mathrm{H})$, $3.42(\mathrm{~s}, 3 \mathrm{H}) 3.14(\mathrm{ddd}, J=6.0,3.2,2.4 \mathrm{~Hz}, 1 \mathrm{H}), 2.00(\mathrm{~d}, J=1.5 \mathrm{~Hz}$, $3 \mathrm{H}) ;{ }^{13} \mathrm{C}\{1 \mathrm{H}\} \mathrm{NMR}\left(100 \mathrm{MHz}, \mathrm{CDCl}_{3}\right) \delta: 133.1,131.5,128.5,128.3$, $124.9,122.9,94.5,87.3,72.5,59.2,58.5,54.1,23.4$.

Synthesis of (Z)-1f and (Z)-1g. To the dry $\mathrm{Et}_{2} \mathrm{O}(15 \mathrm{~mL})$ solution of S5b $(1.1 \mathrm{~g}, 5 \mathrm{mmol})$ was added an ethereal $(15 \mathrm{~mL})$ solution of 2.1 eq. MeMgI $(5.25 \mathrm{~mL}, 10.5 \mathrm{mmol}, 2 \mathrm{M})$ dropwise at $-50{ }^{\circ} \mathrm{C}$, and then the mixture stirred for $6 \mathrm{~h}$ at the same temperature. The mixture was allowed to warm to $0{ }^{\circ} \mathrm{C}$ and quenched by the addition of $30 \mathrm{~mL}$ of saturated $\mathrm{NH}_{4} \mathrm{Cl}(\mathrm{aq})$ solution before extracting with $\mathrm{Et}_{2} \mathrm{O}$. The combined extracts were dried over $\mathrm{MgSO}_{4}$, filtered, and evaporated. The resulting residue was purified on a silica gel column to afford S8 (hexane-EtAc, yield: $0.49 \mathrm{~g}, 80 \%$ ).

The epoxidation of $\mathbf{S 8}(412 \mathrm{mg}, 2 \mathrm{mmol})$ and isolation of the product $(Z)$-1f were performed as specified for $(Z)$-1i (hexane-EtOAc, yield: $0.27 \mathrm{~g}, 60 \%)$.

The hydroxyl group of $(Z)-1 \mathrm{f}(222 \mathrm{mg}, 1.0 \mathrm{mmol})$ was methylated as described above furnishing the enyne oxirane $(Z)-1 \mathrm{~g}$ in $90 \%$ yield $(0.21 \mathrm{~g})$.

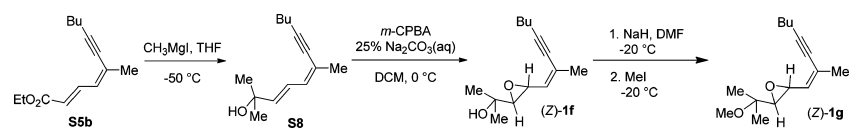

(Z)-1f: ${ }^{1} \mathrm{H}$ NMR $\left(400 \mathrm{MHz}, \mathrm{CDCl}_{3}\right) \delta: 5.26(\mathrm{dd}, J=8.8,1.6 \mathrm{~Hz}$, $1 \mathrm{H}), 3.89(\mathrm{dd}, J=8.8,2.3 \mathrm{~Hz}, 1 \mathrm{H}), 2.88(\mathrm{~d}, J=2.3 \mathrm{~Hz}, 1 \mathrm{H}), 2.36(\mathrm{t}$, $J=7.0 \mathrm{~Hz}, 2 \mathrm{H}), 1.87(\mathrm{~d}, J=1.6 \mathrm{~Hz}, 3 \mathrm{H}), 1.78(\mathrm{~d}, J=0.8 \mathrm{~Hz}, 1 \mathrm{H})$, $1.57-1.40(\mathrm{~m}, 4 \mathrm{H}), 1.31(\mathrm{~s}, 3 \mathrm{H}), 1.25(\mathrm{~s}, 3 \mathrm{H}), 0.92(\mathrm{t}, J=6.8 \mathrm{~Hz}$, $3 \mathrm{H}) ;{ }^{13} \mathrm{C}\{1 \mathrm{H}\} \mathrm{NMR}\left(100 \mathrm{MHz}, \mathrm{CDCl}_{3}\right) \delta: 132.0,125.1,78.8,73.8$, 64.7, 53.3, 50.7, 30.7, 23.7, 22.2, 21.9, 21.0, 19.1, 13.5 .

(Z)-1g: ${ }^{1} \mathrm{H}$ NMR $\left(400 \mathrm{MHz}, \mathrm{CDCl}_{3}\right) \delta: 5.24(\mathrm{dd}, J=8.9,1.5 \mathrm{~Hz}$, $1 \mathrm{H}), 3.69(\mathrm{dd}, J=8.9,2.3 \mathrm{~Hz}, 1 \mathrm{H}), 3.29(\mathrm{~s}, 3 \mathrm{H}), 2.87(\mathrm{~d}, J=2.3 \mathrm{~Hz}$, $1 \mathrm{H}), 2.36(\mathrm{t}, J=6.7 \mathrm{~Hz}, 2 \mathrm{H}), 1.87(\mathrm{~d}, J=1.5 \mathrm{~Hz}, 3 \mathrm{H}), 1.56-1.39(\mathrm{~m}$, $4 \mathrm{H}), 1.18(\mathrm{~s}, 3 \mathrm{H}), 1.15(\mathrm{~s}, 3 \mathrm{H}), 0.92(\mathrm{t}, J=7.2 \mathrm{~Hz}, 3 \mathrm{H}) ;{ }^{13} \mathrm{C}\{1 \mathrm{H}\}$ NMR $\left(100 \mathrm{MHz}, \mathrm{CDCl}_{3}\right) \delta: 132.2,125.3,95.8,79.0,74.0,64.9,53.4$, $50.9,30.9,23.9,22.4,22.0,21.1,19.2,13.7$.

Synthesis of (Z)-1j. To a stirred solution of diphenylethylphosphine oxide $(4.6 \mathrm{~g}, 20 \mathrm{mmol})$ in dry THF $(70 \mathrm{~mL})$ was added BuLi $(2.5 \mathrm{M}$ in hexane, $8.8 \mathrm{~mL}$ ) dropwise at $0{ }^{\circ} \mathrm{C}$ and stirred for a further $30 \mathrm{~min}$. The solution was cooled to $-78{ }^{\circ} \mathrm{C}$, and then the dienyne ester S5b $(3.88 \mathrm{~g}, 20 \mathrm{~mol})$ was added dropwise. The solution was allowed to warm to ambient temperature and subsequently stirred overnight. Saturated $\mathrm{NH}_{4} \mathrm{Cl}$ solution was added, and subsequently its THF content was removed under reduced pressure. The aqueous residue was diluted with brine $(20 \mathrm{~mL})$ and extracted with DCM. The combined organic phases were dried over $\mathrm{MgSO}_{4}$, filtered, and concentrated under reduced pressure. The product $\mathbf{S 9}$ was purified by column chromatography on silica gel (hexane-EtAc, yield: $1.9 \mathrm{~g}, 25 \%) .^{24}$

To a stirred solution of $\mathbf{S 9}(1.9 \mathrm{~g}, 5 \mathrm{mmol})$ in ethanol $(50 \mathrm{~mL})$ was added $\mathrm{NaBH}_{4}(189 \mathrm{mg}, 5 \mathrm{mmol})$ in one portion and stirred for a further $8 \mathrm{~h}$ at ambient temperature. The reaction afforded $\mathbf{S 1 0}$ enriched in threo form. Saturated $\mathrm{NH}_{4} \mathrm{Cl}(15 \mathrm{~mL})$ was added, and subsequently its ethanol content was removed under reduced pressure. The aqueous mixture was diluted with brine $(20 \mathrm{~mL})$ and extracted with DCM. The combined organic layers were dried over $\mathrm{MgSO}_{4}$, filtered, and concentrated under reduced pressure. The product $\mathbf{S 1 0}$ was isolated in pure threo form by column chromatography on silica gel (hexane/EtAc, yield: $1.31 \mathrm{~g}, 69 \%){ }^{24}$

To a stirred solution of $\mathbf{S 1 0}(1.31 \mathrm{~g}, 3.45 \mathrm{mmol})$ in DMF $(50 \mathrm{~mL})$ was added $\mathrm{NaH}$ ( $60 \%$ dispersion in oil; $138 \mathrm{mg}, 3.45 \mathrm{mmol}$ ) in one portion at ambient temperature and stirred for a further $3 \mathrm{~h}$. The reaction was quenched by the addition of $25 \mathrm{~mL}$ of water and $15 \mathrm{~mL}$ of brine and subsequently extracted with $\mathrm{Et}_{2} \mathrm{O}$. The combined extracts were washed with water, dried over $\mathrm{MgSO}_{4}$, filtered, and concentrated under reduced pressure. The product $\mathbf{S} \mathbf{3} \mathbf{j}$ was purified by column chromatography on silica gel (hexane, yield: $330 \mathrm{mg}, 59 \%){ }^{24}$ 
The epoxidation of S3j $(162 \mathrm{mg}, 1 \mathrm{mmol})$ and isolation of the product $(Z)-\mathbf{1} \mathbf{j}$ were performed as specified for $(Z)-\mathbf{1 i}$ (hexane-EtAc, yield: $35.6 \mathrm{mg}, 20 \%)$.
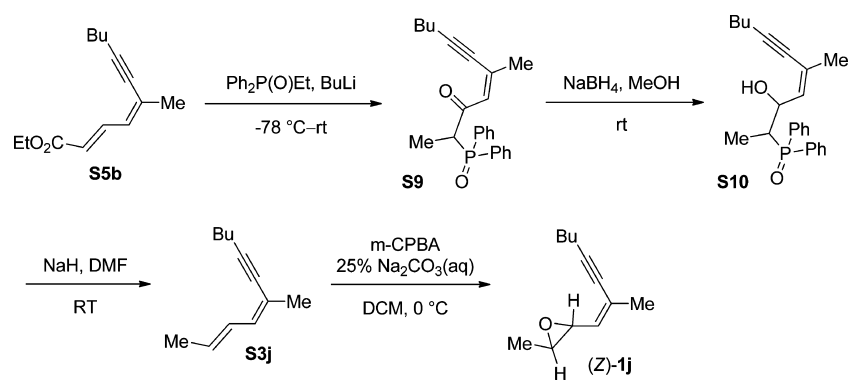

S9: ${ }^{1} \mathrm{H}$ NMR $\left(400 \mathrm{MHz}, \mathrm{CDCl}_{3}\right) \delta: 7.86-7.74(\mathrm{~m}, 4 \mathrm{H}), 7.55-7.43$ $(\mathrm{m}, 6 \mathrm{H}), 6.34(\mathrm{~d}, J=1.2 \mathrm{~Hz}, 1 \mathrm{H}), 3.99(\mathrm{dq}, J=13.5,7.0 \mathrm{~Hz}, 1 \mathrm{H}), 2.42$ $(\mathrm{t}, J=7.0 \mathrm{~Hz}, 2 \mathrm{H}), 1.89(\mathrm{~d}, J=1.2 \mathrm{~Hz}, 3 \mathrm{H}), 1.60-1.51(\mathrm{~m}, 2 \mathrm{H})$, $1.47-1.38(\mathrm{~m}, 5 \mathrm{H}), 0.92(\mathrm{t}, J=7.0 \mathrm{~Hz}, 3 \mathrm{H})$.

S10: ${ }^{1} \mathrm{H}$ NMR (400 MHz, $\left.\mathrm{CDCl}_{3}\right) \delta: 7.83-7.71(\mathrm{~m}, 4 \mathrm{H}), 7.56-$ $7.43(\mathrm{~m}, 6 \mathrm{H}), 5.53(\mathrm{dd}, J=8.3,1.2 \mathrm{~Hz}, 1 \mathrm{H}), 4.80(\mathrm{dt}, J=13.8,8.3 \mathrm{~Hz}$, $1 \mathrm{H}), 2.82-2.71(\mathrm{~m}, 1 \mathrm{H}), 2.15(\mathrm{t}, J=7.2 \mathrm{~Hz}, 2 \mathrm{H}), 1.65(\mathrm{~d}, J=1.2 \mathrm{~Hz}$, $3 \mathrm{H}), 1.40-1.28(\mathrm{~m}, 4 \mathrm{H}), 1.04(\mathrm{dd}, J=17.2,7.4 \mathrm{~Hz}, 3 \mathrm{H}), 0.78(\mathrm{t}, J=$ $7.2 \mathrm{~Hz}, 3 \mathrm{H})$

S3j: ${ }^{1} \mathrm{H}$ NMR $\left(400 \mathrm{MHz}, \mathrm{CDCl}_{3}\right) \delta: 6.48(\mathrm{ddq}, J=15.4,10.8,1.6$ $\mathrm{Hz}, 1 \mathrm{H}), 6.11(\mathrm{~d}, J=10.8 \mathrm{~Hz}, 1 \mathrm{H}), 4.80(\mathrm{dq}, J=15.4,7.0 \mathrm{~Hz}, 1 \mathrm{H})$, $2.39(\mathrm{t}, J=6.8 \mathrm{~Hz}, 2 \mathrm{H}), 1.86(\mathrm{~s}, 3 \mathrm{H}), 1.79(\mathrm{~d}, J=7.0 \mathrm{~Hz}, 3 \mathrm{H}), 1.60-$ $1.52(\mathrm{~m}, 2 \mathrm{H}), 1.51-1.44(\mathrm{~m}, 2 \mathrm{H}), 0.94(\mathrm{t}, J=6.8 \mathrm{~Hz}, 3 \mathrm{H})$.

(Z)-1j: ${ }^{1} \mathrm{H}$ NMR $\left(400 \mathrm{MHz}, \mathrm{C}_{6} \mathrm{D}_{6}\right) \delta: 5.16(\mathrm{dd}, J=8.9,1.4 \mathrm{~Hz}$, $1 \mathrm{H}), 3.75(\mathrm{dd}, J=8.9,2.1 \mathrm{~Hz}, 1 \mathrm{H}), 2.64(\mathrm{qd}, J=5.4,2.1 \mathrm{~Hz}, 1 \mathrm{H}), 2.08$ $(\mathrm{t}, J=6.8 \mathrm{~Hz}, 2 \mathrm{H}), 1.73(\mathrm{~d}, J=1.4 \mathrm{~Hz}, 3 \mathrm{H}), 1.31-1.20(\mathrm{~m}, 4 \mathrm{H}), 1.04$ $(\mathrm{d}, J=5.2 \mathrm{~Hz}, 3 \mathrm{H}), 0.72(\mathrm{t}, J=7.2 \mathrm{~Hz}, 3 \mathrm{H}) ;{ }^{13} \mathrm{C}\{1 \mathrm{H}\}$ NMR $(100$ $\left.\mathrm{MHz}, \mathrm{C}_{6} \mathrm{D}_{6}\right) \delta: 133.7,123.9,95.2,79.3,57.4,55.2,30.6,23.4,21.8$, 19.0, 17.3, 13.2.

Synthesis of (Z)-1t. $\mathrm{PBr}_{3}(1.4 \mathrm{~mL}, 13.8 \mathrm{mmol})$ was added dropwise to a mixture of DMF $(1.2 \mathrm{~mL}, 15.3 \mathrm{mmol})$ and chloroform $(10 \mathrm{~mL})$ at $0{ }^{\circ} \mathrm{C}$, and then the resulting mixture stirred for $1 \mathrm{~h}$. Subsequently, $0.5 \mathrm{~g}$ of cyclohexanone $(6 \mathrm{mmol})$ was added dropwise and stirred for $8 \mathrm{~h}$ at room temperature. The reaction was terminated with water, neutralized with the addition of solid $\mathrm{NaHCO}_{3}$, and extracted with DCM. The extract was washed with brine, dried over $\mathrm{MgSO}_{4}$, filtered, and concentrated under reduced pressure. The purification on short silica gel column provided the compound S11 (hexane/EtAC, $0.92 \mathrm{~g}, 81 \%$ ).

A mixture of $S 11$ (945 mg, $5 \mathrm{mmol}), \mathrm{Pd}\left(\mathrm{PPh}_{3}\right)_{4}(123 \mathrm{mg}, 0.1 \mathrm{mmol}$, $2 \mathrm{~mol} \mathrm{\%}$ of $\mathrm{Pd})$, and $\mathrm{CuI}(21 \mathrm{mg}, 0.1 \mathrm{mmol}, 2 \mathrm{mmol} \%$ of $\mathrm{Cu})$ in $10 \mathrm{~mL}$ of $\mathrm{Et}_{3} \mathrm{~N}$ was stirred for $10 \mathrm{~min}$ at room temperature followed by the addition of 1-hexyne $(0.5 \mathrm{~g}, 6 \mathrm{mmol})$. After being stirred for $3 \mathrm{~h}$ at room temperature, water was added and extracted with $\mathrm{Et}_{2} \mathrm{O}$. The combined organic phases were dried over $\mathrm{MgSO}_{4}$, filtered, and concentrated under reduced pressure. The residue was purified by column chromatography on silica gel to obtain endocyclic enyne aldehyde S4t (hexane/EtAc, $0.84 \mathrm{~g}, 90 \%)^{25}$

The conversion of $\mathbf{S 4 t}(840 \mathrm{mg}, 4.42 \mathrm{mmol})$ to dienyne ester $\mathbf{S 5 t}$ was performed by HWE reaction as described above (hexane/EtAc, $0.96 \mathrm{~g}, 84 \%$ ). Further successive synthetic procedures, which involved the reduction of the ester S5t $(960 \mathrm{mg}, 3.7 \mathrm{mmol}$ ) to the enyne alcohol S6t $(730 \mathrm{mg}, 91 \%$ yield), the epoxidation of S6t $(436 \mathrm{mg}$, $2 \mathrm{mmol}$ ) to $\mathbf{S 7 t}$ ( $260 \mathrm{mg}$, $55 \%$ yield), and finally methyl derivatization of hydroxyl group of $\mathbf{S 7 t}(260 \mathrm{mg}, 1.1 \mathrm{mmol})$ to obtain $(Z)-\mathbf{1 t}(0.22 \mathrm{~g}$, $90 \%)$ were all conducted as described above.

(Z)-1t: ${ }^{1} \mathrm{H}$ NMR $\left(400 \mathrm{MHz}, \mathrm{CDCl}_{3}\right) \delta: 4.00-3.97(\mathrm{~m}, 1 \mathrm{H}), 3.73$ $(\mathrm{dt}, J=11.2,2.8 \mathrm{~Hz}, 1 \mathrm{H}), 3.45-3.30(\mathrm{~m}, 4 \mathrm{H}), 3.20$ (sext, $J=2.8 \mathrm{~Hz}$, $1 \mathrm{H}), 2.34(\mathrm{t}, J=6.8 \mathrm{~Hz}, 2 \mathrm{H}), 2.21-2.10(\mathrm{~m}, 2 \mathrm{H}), 2.02-1.91(\mathrm{~m}, 2 \mathrm{H})$, $1.79-1.32(\mathrm{~m}, 6 \mathrm{H}), 0.98-0.79(\mathrm{~m}, 5 \mathrm{H}) ;{ }^{13} \mathrm{C}\{1 \mathrm{H}\}$ NMR $(100 \mathrm{MHz}$, $\left.\mathrm{CDCl}_{3}\right) \delta: 137.3,121.7,94.2,79.5,73.1,59.2,56.2,55.2,30.9,30.8$, 22.4, 22.2, 21.9, 21.7, 19.2, 13.6 .

Carbonylation Reactions. A palladium complex, phosphine ligand, and $1 \mathrm{~mL}$ of alcohol were added successively to a Schlenck apparatus that is attached to an $\mathrm{Ar}$ line and stirred $1 \mathrm{~h}$ at $25^{\circ} \mathrm{C}$ before the addition of the substrate. A CO full balloon was then fixed to the
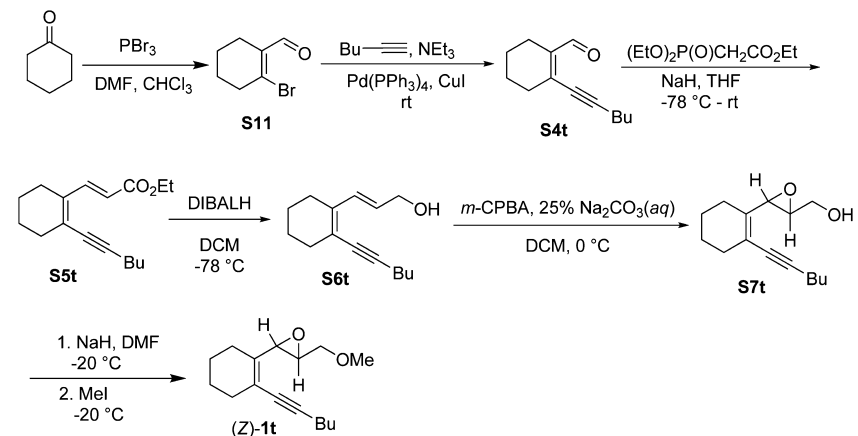

reaction vessel, and $1 \mathrm{~mL}$ of alcohol solution of the substrate $\mathbf{1}$ or $\mathbf{1}^{\prime}$ was added. When the reaction was complete as judged by TLC analysis, the solvent content was evaporated and the residue was purified by column chromatography on silica gel to afford $\mathbf{2}$ or $\mathbf{2}^{\prime}$ as a pale yellow oil. The coupling constants of olefinic protons and NOE studies confirmed (E)-configured structures. ${ }^{1} \mathrm{H}$ NMR analyses of vinylallene products, except that of $2 \mathbf{i} \mathbf{a}^{\prime}$, were performed in $\mathrm{C}_{6} \mathrm{D}_{6}$. With this solvent, the ${ }^{1} \mathrm{H}$ NMR signals of diastereomers were resolved adequately, allowing to determine diastereomeric ratios smoothly. In contrast, when using $\mathrm{CDCl}_{3}$ solvent, diastereomeric signals were all overlapped.

Product Data. 2aa: Yield: $24.9 \mathrm{mg}$ (99\%); ${ }^{1} \mathrm{H}$ NMR (400 MHz, $\left.\mathrm{CDCl}_{3}\right) \delta: 6.13(\mathrm{~d}, J=16.0 \mathrm{~Hz}, 1 \mathrm{H}), 5.76(\mathrm{~d}, J=16.0 \mathrm{~Hz}, 1 \mathrm{H}), 3.67$ $(\mathrm{s}, 3 \mathrm{H}), 2.22(\mathrm{t}, J=7.6 \mathrm{~Hz}, 2 \mathrm{H}), 1.85(\mathrm{~s}, 3 \mathrm{H}), 1.37-1.27(\mathrm{~m}, 10 \mathrm{H})$, $0.86(\mathrm{t}, J=7.0 \mathrm{~Hz}, 3 \mathrm{H}) ;{ }^{13} \mathrm{C}\{1 \mathrm{H}\} \mathrm{NMR}\left(100 \mathrm{MHz}, \mathrm{CDCl}_{3}\right) \delta: 213.4$, 167.7, 137.8, 122.9, 103.1, 99.7, 70.9, 52.0, 30.1, 29.8, 28.6, 22.1, 14.8, 13.8; FTIR $\left(\nu_{\max } / \mathrm{cm}^{-1}\right): 3409,2957,2930,2871,1940,1714,1436$, $1378,1264,1133,1083,1019,966,912,723,696$; MS (EI, $m / z): 252$ $\left(1, \mathrm{M}^{+}\right), 234(2), 205(7), 194(7), 179(5), 151(23), 135(35), 121$ (15), 107 (32), 91 (43), 77 (25), 65 (15), 59 (46), 43 (100); HRMS (ESI) $\mathrm{C}_{15} \mathrm{H}_{25} \mathrm{O}_{3}(\mathrm{MH})^{+}, 253.1798$ (calculated); 253.1799 (found).

2ba: Yield: $25.2 \mathrm{mg}(94 \%) ;{ }^{1} \mathrm{H}$ NMR $\left(400 \mathrm{MHz}, \mathrm{C}_{6} \mathrm{D}_{6}\right) \delta: 6.43$ (dd, $J=15.7,1.6 \mathrm{~Hz}, 1 \mathrm{H}$, major), 6.41 (dd, $J=15.7,1.6 \mathrm{~Hz}, 1 \mathrm{H}$, minor), 5.51 (dd, $J=15.7,5.5 \mathrm{~Hz}, 1 \mathrm{H}$, major), 5.51 (dd, $J=15.7,5.5$ $\mathrm{Hz}, 1 \mathrm{H}$, minor), 4.23-4.19 (m, $1 \mathrm{H}), 3.36$ (s, 3H, major), 3.35 (s, $3 \mathrm{H}$, minor), $3.03\left(\mathrm{dd}, \mathrm{A}\right.$ of $\left.\mathrm{ABX}, J_{\mathrm{AB}}=9.2 \mathrm{~Hz}, J_{\mathrm{AX}}=4.0 \mathrm{~Hz}, 1 \mathrm{H}\right), 2.98(\mathrm{dd}$, $\mathrm{B}$ of $\left.\mathrm{ABX}, J_{\mathrm{AB}}=9.2 \mathrm{~Hz}, J_{\mathrm{BX}}=7.2 \mathrm{~Hz}, 1 \mathrm{H}\right), 2.95(\mathrm{~s}, 3 \mathrm{H}), 2.39(\mathrm{td}, J=$ 7.5, $2.2 \mathrm{~Hz}, 2 \mathrm{H}), 2.23(\mathrm{bs}, 1 \mathrm{H}), 1.69(\mathrm{~s}, 3 \mathrm{H}), 1.47-1.39(\mathrm{~m}, 2 \mathrm{H}), 1.23$ (sext, $J=7.6 \mathrm{~Hz}, 2 \mathrm{H}), 0.78(\mathrm{t}, J=7.2 \mathrm{~Hz}, 3 \mathrm{H}$, major), $0.78(\mathrm{t}, J=7.2$ $\mathrm{Hz}, 3 \mathrm{H}$, minor $) ;{ }^{13} \mathrm{C}\{1 \mathrm{H}\}$ NMR: $\left(100 \mathrm{MHz}, \mathrm{C}_{6} \mathrm{D}_{6}\right) \delta: 213.5,166.9$, 129.2, 127.3, 103.1, 76.5, 70.6, 58.2, 51.3, 30.3, 28.9, 22.2, 14.5, 13.7; FTIR $\left(\nu_{\max } / \mathrm{cm}^{-1}\right): 3419,2955,2928,2870,1943,1715,1456,1436$, 1379, 1266, 1241, 1193, 1129, 1016, 966; MS (EI, $m / z): 268\left(1, \mathrm{M}^{+}\right)$, 236 (1), 223 (4), 205 (1), 191 (6), 151 (2), 135 (7), 121 (6), 93 (8), 91 (14), 77 (10), 59 (6), 45 (100); HRMS (ESI): $\mathrm{C}_{15} \mathrm{H}_{25} \mathrm{O}_{4}(\mathrm{MH})^{+}$, 269.1747 (calculated); 269.1747 (found).

2ba': Yield: $23.3 \mathrm{mg},(87 \%) ;{ }^{1} \mathrm{H}$ NMR (400 MHz, $\left.\mathrm{C}_{6} \mathrm{D}_{6}\right) \delta: 6.42$ (dd, $J=15.7,1.6 \mathrm{~Hz}, 1 \mathrm{H}$, major), 6.40 (dd, $J=15.7,1.6 \mathrm{~Hz}, 1 \mathrm{H}$, minor), $5.52(\mathrm{dd}, J=15.7,5.7 \mathrm{~Hz}, 1 \mathrm{H}), 4.22-4.20(\mathrm{~m}, 1 \mathrm{H}), 3.37(\mathrm{~s}$, $3 \mathrm{H}$, major), 3.35 (s, $3 \mathrm{H}$, minor), 3.03 (dd, $\mathrm{A}$ of $\mathrm{ABX}, J_{\mathrm{AB}}=9.6 \mathrm{~Hz}$, $\left.J_{\mathrm{AX}}=4.0 \mathrm{~Hz}, 1 \mathrm{H}\right), 2.99\left(\mathrm{dd}, \mathrm{B}\right.$ of $\left.\mathrm{ABX}, J_{\mathrm{AB}}=9.6 \mathrm{~Hz}, J_{\mathrm{BX}}=8.0 \mathrm{~Hz}, 1 \mathrm{H}\right)$, $2.96(\mathrm{~s}, 3 \mathrm{H}), 2.38(\mathrm{td}, J=7.6 \mathrm{~Hz}, 1.8 \mathrm{~Hz}, 2 \mathrm{H}), 2.29$ (bs, $1 \mathrm{H}), 1.70(\mathrm{~s}$, $3 \mathrm{H}), 1.48-1.40(\mathrm{~m}, 2 \mathrm{H}), 1.25(\mathrm{sext}, J=7.6 \mathrm{~Hz}, 2 \mathrm{H}), 0.79(\mathrm{t}, J=7.6$ $\mathrm{Hz}, 3 \mathrm{H}) ;{ }^{13} \mathrm{C}\{1 \mathrm{H}\}$ NMR $\left(100 \mathrm{MHz}, \mathrm{C}_{6} \mathrm{D}_{6}\right) \delta: 213.5,166.9,129.2$, 103.1, 100.0, 76.5, 70.6, 58.2, 51.3, 30.3, 28.9, 22.2, 14.5, 13.7; FTIR $\left(\nu_{\max } / \mathrm{cm}^{-1}\right): 3419,2955,2928,2870,1943,1715,1456,1436,1379$, 1266, 1241, 1193, 1129, 1016, 966; MS (EI, $m / z): 268\left(1, \mathrm{M}^{+}\right), 236$ (1), 223 (4), 205 (1), 191 (6), 151 (2), 135 (7), 121 (6), 93 (8), 91 (14), 77 (10), 59 (6), 45 (100); HRMS (ESI): $\mathrm{C}_{15} \mathrm{H}_{25} \mathrm{O}_{4}(\mathrm{MH})^{+}$, 269.1747 (calculated); 269.1747 (found).

2bb: Yield: $23.7 \mathrm{mg}(84 \%) ;{ }^{1} \mathrm{H}$ NMR (400 MHz, $\left.\mathrm{C}_{6} \mathrm{D}_{6}\right) \delta: 6.44$ (dd, $J=15.4,1.6 \mathrm{~Hz}, 1 \mathrm{H}$, major), $6.43(\mathrm{dd}, J=15.4,1.6 \mathrm{~Hz}, 1 \mathrm{H}$, minor), 5.54 (dd, $J=15.4,5.6 \mathrm{~Hz}, 1 \mathrm{H}), 4.24-4.18(\mathrm{~m}, 1 \mathrm{H}), 3.99$ $(\mathrm{q}, J=7.1 \mathrm{~Hz}, 2 \mathrm{H}), 3.03\left(\mathrm{dd}, \mathrm{A}\right.$ of $\mathrm{ABX}, J_{\mathrm{AB}}=9.6 \mathrm{~Hz}, J_{\mathrm{AX}}=4.0 \mathrm{~Hz}$, $1 \mathrm{H}), 2.98\left(\mathrm{dd}, \mathrm{B}\right.$ of $\left.\mathrm{ABX}, J_{\mathrm{AB}}=9.6 \mathrm{~Hz}, J_{\mathrm{BX}}=7.6 \mathrm{~Hz}, 1 \mathrm{H}\right), 2.95(\mathrm{~s}, 3 \mathrm{H})$, $2.41(\mathrm{td}, J=7.2,2.4 \mathrm{~Hz}, 2 \mathrm{H}) 2.28$ (bs, $1 \mathrm{H}), 1.71(\mathrm{~s}, 3 \mathrm{H}), 1.49-1.41$ 
(m, 2H), 1.25 (sext, $J=7.2 \mathrm{~Hz}, 2 \mathrm{H}), 0.92(\mathrm{t}, J=7.1 \mathrm{~Hz}, 3 \mathrm{H}$, major), 0.91 $(\mathrm{t}, J=7.1 \mathrm{~Hz}, 3 \mathrm{H}$, minor) $0.79(\mathrm{t}, J=7.2 \mathrm{~Hz}, 3 \mathrm{H}$, major), $0.79(\mathrm{t}, J=$ $7.2 \mathrm{~Hz}, 3 \mathrm{H}$, minor $) ;{ }^{13} \mathrm{C}\{1 \mathrm{H}\}$ NMR: $\left(100 \mathrm{MHz}, \mathrm{CDCl}_{3}\right) \delta: 213.5,167.1$, 128.7, 127.8, 103.1, 100.3, 76.4, 71.1, 60.8, 59.1, 30.2, 28.6, 22.2, 14.7, 14.3, 13.9; FTIR $\left(\nu_{\max } / \mathrm{cm}^{-1}\right): 3418,2956,2926,2858,1940,1708,1458$, $1367,1322,1261,1240,1172,1118,1062,1023,965,864,713$. MS (EI, $m / z): 282\left(1, \mathrm{M}^{+}\right), 238$ (1), 237 (4), 209 (2), 191 (5), 135 (3), 121 (5), 107 (6), 93 (8), 91 (12), 77 (7), 65 (5), 55 (7), 45 (100); HRMS (ESI): $\mathrm{C}_{16} \mathrm{H}_{26} \mathrm{O}_{4}(\mathrm{MH})^{+}, 283.1904$ (calculated); 283.1909 (found).

2bc: Yield: $25.8 \mathrm{mg}(87 \%) ;{ }^{1} \mathrm{H}$ NMR $\left(400 \mathrm{MHz}, \mathrm{C}_{6} \mathrm{D}_{6}\right) \delta: 6.44(\mathrm{dd}$, $J=15.8,1.6 \mathrm{~Hz}, 1 \mathrm{H}$, major), 6.43 (dd, $J=15.8,1.6 \mathrm{~Hz}, 1 \mathrm{H}$, minor), $5.53(\mathrm{dd}, J=15.8,5.6 \mathrm{~Hz}, 1 \mathrm{H}), 4.23-4.19(\mathrm{~m}, 1 \mathrm{H}), 4.02-3.92(\mathrm{~m}$, $2 \mathrm{H}), 3.03\left(\mathrm{dd}, \mathrm{A}\right.$ of $\left.\mathrm{ABX}, J_{\mathrm{AB}}=9.6 \mathrm{~Hz}, J_{\mathrm{AX}}=4.4 \mathrm{~Hz}, 1 \mathrm{H}\right), 2.98(\mathrm{dd}, \mathrm{B}$ of $\left.\mathrm{ABX}, J_{\mathrm{AB}}=9.6 \mathrm{~Hz}, J_{\mathrm{BX}}=7.2 \mathrm{~Hz}, 1 \mathrm{H}\right), 2.94(\mathrm{~s}, 3 \mathrm{H}), 2.41(\mathrm{td}, J=7.3$, $1.6 \mathrm{~Hz}, 2 \mathrm{H}), 2.22(\mathrm{bs}, 1 \mathrm{H}), 1.72(\mathrm{~s}, 3 \mathrm{H}), 1.49-1.33(\mathrm{~m}, 4 \mathrm{H}), 1.25$ (sext, $J=7.4 \mathrm{~Hz}, 2 \mathrm{H}), 0.79(\mathrm{t}, J=7.4 \mathrm{~Hz}, 3 \mathrm{H}$, major), $0.80(\mathrm{t}, J=7.4$ $\mathrm{Hz}, 3 \mathrm{H}$, minor), $0.66(\mathrm{t}, J=7.3 \mathrm{~Hz}, 3 \mathrm{H}$, major), $0.65(\mathrm{t}, J=7.3 \mathrm{~Hz}$, $3 \mathrm{H}$, minor); ${ }^{13} \mathrm{C}\{1 \mathrm{H}\}$ NMR (100 $\left.\mathrm{MHz}, \mathrm{CDCl}_{3}\right) \delta: 213.6,167.2$, 128.6, 127.9, 103.1, 100.3, 76.5, 71.1, 66.3, 59.1, 30.2, 28.5, 22.2, 22.0, 14.7, 13.9, 10.3; FTIR $\left(\nu_{\max } / \mathrm{cm}^{-1}\right): 3406,2958,2928,1941,1709$, 1458, 1397, 1322, 1263, 1172, 1132, 1062, 1015, 965, 713; MS (EI, $m / z): 296\left(1, \mathrm{M}^{+}\right), 278$ (1), 251 (3), 236 (1), 209 (3), 191 (5), 135 (4), 121 (5), 91 (10), 79 (6), 65 (3), 45 (100); HRMS (ESI): $\mathrm{C}_{17} \mathrm{H}_{28} \mathrm{O}_{4}(\mathrm{MH})^{+}, 297.2060$ (calculated), 297.2065 (found).

2bd: Yield: $25.7 \mathrm{mg}(83 \%) ;{ }^{1} \mathrm{H}$ NMR (400 MHz, $\left.\mathrm{C}_{6} \mathrm{D}_{6}\right) \delta: 6.45$ (dd, $J=16.0,1.2 \mathrm{~Hz}, 1 \mathrm{H}$, major), $6.43(\mathrm{dd}, J=16.0,1.2 \mathrm{~Hz}, 1 \mathrm{H}$, minor), 5.53 (dd, $J=16.0,5.6 \mathrm{~Hz}, 1 \mathrm{H}$, major), 5.53 (dd, $J=16,5.6$ $\mathrm{Hz}, 1 \mathrm{H}$, minor), 4.24-4.19 (m, 1H), 4.09-3.99 (m, 2H), 3.04 (dd, A of $\left.\mathrm{ABX}, J_{\mathrm{AB}}=9.6 \mathrm{~Hz}, J_{\mathrm{AX}}=4.0 \mathrm{~Hz}, 1 \mathrm{H}\right), 2.99\left(\mathrm{dd}, \mathrm{B}\right.$ of $\mathrm{ABX}, J_{\mathrm{AB}}=9.6$ $\left.\mathrm{Hz}, J_{\mathrm{BX}}=7.6 \mathrm{~Hz}, 1 \mathrm{H}\right), 2.95(\mathrm{~s}, 3 \mathrm{H}), 2.41(\mathrm{td}, J=7.4,2.4 \mathrm{~Hz}, 2 \mathrm{H}) 2.26$ (bs, $1 \mathrm{H}), 1.75$ (s, 3H, minor), 1.73 (s, 3H, major), 1.46 (quint, $J=7.2$ $\mathrm{Hz}, 2 \mathrm{H}$ ), 1.36 (quint, $J=7.4 \mathrm{~Hz}, 2 \mathrm{H}), 1.25$ (sext, $J=7.2 \mathrm{~Hz}, 2 \mathrm{H}), 1.12$ (sext, $J=7.4 \mathrm{~Hz}, 2 \mathrm{H}), 0.80(\mathrm{t}, J=7.2 \mathrm{~Hz}, 3 \mathrm{H}$, minor), $0.79(\mathrm{t}, J=7.2$ $\mathrm{Hz}, 3 \mathrm{H}$, major), $0.68(\mathrm{t}, J=7.4 \mathrm{~Hz}, 3 \mathrm{H}$, major), $0.67(\mathrm{t}, J=7.4 \mathrm{~Hz}, 3 \mathrm{H}$, minor); ${ }^{13} \mathrm{C}\{1 \mathrm{H}\}$ NMR $\left(100 \mathrm{MHz}, \mathrm{CDCl}_{3}\right) \delta: 213.6,167.2,128.6$, $127.8,103.1,100.3,76.4,71.1,64.7,59.1,30.6,30.2,28.5,22.2,19.2$, 14.7, 13.9, 13.7; FTIR $\left(\nu_{\max } / \mathrm{cm}^{-1}\right): 3428,2958,2929,2873,1940$, 1710, 1458, 1379, 1322, 1261, 1131, 1062, 1016, 965, 713; MS (EI, $m / z)$ : $310\left(1, \mathrm{M}^{+}\right), 293$ (1), 265 (1), 235 (2), 207 (4), 191 (4), 161 (3), 121 (3), 105 (5), 91 (10), 79 (5), 57 (15), 45 (100); HRMS (ESI): $\mathrm{C}_{18} \mathrm{H}_{31} \mathrm{O}_{4}(\mathrm{MH})^{+}, 311.2217$ (calculated), 311.2219 (found).

2be: Yield: $26.1 \mathrm{mg}(88 \%) ;{ }^{1} \mathrm{H}$ NMR $\left(400 \mathrm{MHz}, \mathrm{C}_{6} \mathrm{D}_{6}\right) \delta: 6.43$ (dd, $J=16.0,1.6 \mathrm{~Hz}, 1 \mathrm{H}), 5.51$ (dd, $J=16.0,5.6 \mathrm{~Hz}, 1 \mathrm{H}), 5.05$ (sept, $J=6.4 \mathrm{~Hz}, 1 \mathrm{H}$, major), 5.05 (sept, $J=6.4 \mathrm{~Hz}, 1 \mathrm{H}$, minor), $4.23-4.16$ (m, 1H), $3.02\left(\mathrm{dd}, \mathrm{A}\right.$ of $\left.\mathrm{ABX}, J_{\mathrm{AB}}=9.2 \mathrm{~Hz}, J_{\mathrm{AX}}=4.0 \mathrm{~Hz}, 1 \mathrm{H}\right), 2.97$ (dd, B of $\left.\mathrm{ABX}, J_{\mathrm{AB}}=9.2 \mathrm{~Hz}, J_{\mathrm{BX}}=7.6 \mathrm{~Hz}, 1 \mathrm{H}\right), 2.94(\mathrm{~s}, 3 \mathrm{H}), 2.42(\mathrm{td}$, $J=7.6,2.0 \mathrm{~Hz}, 2 \mathrm{H}), 2.21$ (bs, $1 \mathrm{H}), 1.71(\mathrm{~s}, 3 \mathrm{H}), 1.45$ (quint, $J=7.6$ $\mathrm{Hz}, 2 \mathrm{H}), 1.25$ (sext, $J=7.6 \mathrm{~Hz}, 2 \mathrm{H}), 1.02(\mathrm{~d}, J=6.4 \mathrm{~Hz}, 3 \mathrm{H}), 1.01$ $(\mathrm{d}, J=6.4 \mathrm{~Hz}, 3 \mathrm{H}), 0.79(\mathrm{t}, J=7.6 \mathrm{~Hz}, 3 \mathrm{H}$, major $), 0.79(\mathrm{t}, J=7.6 \mathrm{~Hz}$, $3 \mathrm{H}$, minor); ${ }^{13} \mathrm{C}\{1 \mathrm{H}\}$ NMR (100 MHz, $\left.\mathrm{CDCl}_{3}\right) \delta: 213.5,166.7$, 128.9, 127.6, 103.0, 100.7, 76.5, 71.1, 68.1, 59.1, 30.2, 28.6, 22.2, 21.9, 21.8, 14.7, 13.9; FTIR $\left(\nu_{\max } / \mathrm{cm}^{-1}\right) \delta: 3442,2957,2927,1942,1706$, 1457, 1373, 1322, 1264, 1107, 1062, 1014, 966,831,713; MS (EI, $m / z)$ : $296\left(1, \mathrm{M}^{+}\right), 282$ (1), 251 (2), 209 (3), 191 (4), 180 (1), 121 (6), 107 (4), 91 (7), 77 (6), 55 (4), 45 (100); HRMS (ESI): $\mathrm{C}_{17} \mathrm{H}_{29} \mathrm{O}_{4}(\mathrm{MH})^{+}$, 297.2060 (calculated), 297.2060 (found).

2bg: Yield: $26.8 \mathrm{mg}$ (91\%); ${ }^{1} \mathrm{H}$ NMR (400 MHz, $\left.\mathrm{C}_{6} \mathrm{D}_{6}\right) \delta: 6.43$ (dd, $J=15.6,0.8 \mathrm{~Hz}, 1 \mathrm{H}), 5.72-5.62(\mathrm{~m}, 1 \mathrm{H}), 5.52(\mathrm{dd}, J=15.6,5.6$ $\mathrm{Hz}, 1 \mathrm{H}), 5.08(\mathrm{dq}, J=18.8,1.6 \mathrm{~Hz}, 1 \mathrm{H}), 4.89(\mathrm{dq}, J=6.4,1.6 \mathrm{~Hz}$, $1 \mathrm{H}), 4.53-4.41(\mathrm{~m}, 2 \mathrm{H}), 4.23-4.19(\mathrm{~m}, 1 \mathrm{H}), 3.04(\mathrm{dd}, \mathrm{A}$ of $\mathrm{ABX}$, $\left.J_{\mathrm{AB}}=9.6 \mathrm{~Hz}, J_{\mathrm{AX}}=4.0 \mathrm{~Hz}, 1 \mathrm{H}\right), 2.99\left(\mathrm{dd}, \mathrm{B}\right.$ of $\mathrm{ABX}, J_{\mathrm{AB}}=9.6 \mathrm{~Hz}, J_{\mathrm{BX}}=$ $7.2 \mathrm{~Hz}, 1 \mathrm{H}), 2.95(\mathrm{~s}, 3 \mathrm{H}), 2.39(\mathrm{td}, J=7.2,2.0 \mathrm{~Hz}, 2 \mathrm{H}), 2.31(\mathrm{bs}, 1 \mathrm{H})$, $1.70(\mathrm{~s}, 3 \mathrm{H}), 1.44$ (quint, $J=7.2 \mathrm{~Hz}, 2 \mathrm{H}), 1.24($ sext, $J=7.2 \mathrm{~Hz}, 2 \mathrm{H})$, $0.79(\mathrm{t}, J=7.2 \mathrm{~Hz}, 3 \mathrm{H}$, minor), $0.78(\mathrm{t}, J=7.2 \mathrm{~Hz}, 3 \mathrm{H}$, major); ${ }^{13} \mathrm{C}\{1 \mathrm{H}\}$ NMR $\left(100 \mathrm{MHz}, \mathrm{CDCl}_{3}\right) \delta: 213.7,166.8,132.4,128.5$, 128.1, 117.4, 103.2, 100.0, 76.4, 71.1, 65.2, 59.1, 30.1, 28.5, 22.2, 14.7, 13.9; FTIR $\left(\nu_{\max } / \mathrm{cm}^{-1}\right): 3417,2956,2928,2873,1939,1710,1647$, 1456, 1379, 1322, 1259, 1236, 1172, 1129, 1062, 1015, 965, 928, 837, 713. MS (EI, $m / z$ ): 294 (1, M+), 261 (1), 249 (2), 221 (1), 191 (5),
165 (3), 121 (6), 91 (11), 77 (9), 65 (4), 55 (7), 45 (100); HRMS (ESI): $\mathrm{C}_{17} \mathrm{H}_{27} \mathrm{O}_{4}(\mathrm{MH})^{+}, 295.1904$ (calculated), 295.1910 (found).

2ca: Yield: $31.0 \mathrm{mg}(90 \%) ;{ }^{1} \mathrm{H}$ NMR $\left(400 \mathrm{MHz}, \mathrm{C}_{6} \mathrm{D}_{6}\right) \delta: 7.24-$ $7.01(\mathrm{~m}, 5 \mathrm{H}), 6.44$ (dd, $J=15.8,1.6 \mathrm{~Hz}, 1 \mathrm{H}$, major), 6.42 (dd, $J=$ $15.8,1.6 \mathrm{~Hz}, 1 \mathrm{H}$, minor), 5.49 (dd, $J=15.8,5.7 \mathrm{~Hz}, 1 \mathrm{H}), 4.29-4.23$ $(\mathrm{m}, 1 \mathrm{H}), 4.21\left(\mathrm{~d}, \mathrm{~A}\right.$ of $\left.\mathrm{AB}, J_{\mathrm{AB}}=12.0 \mathrm{~Hz}, 1 \mathrm{H}\right), 4.19\left(\mathrm{~d}, \mathrm{~B}\right.$ of $\mathrm{AB}, J_{\mathrm{AB}}=$ $12.0 \mathrm{~Hz}, 1 \mathrm{H}), 3.36(\mathrm{~s}, 3 \mathrm{H}$, major) $3.34(\mathrm{~s}, 3 \mathrm{H}$, minor), 3.18 (dd, $\mathrm{A}$ of $\left.\mathrm{ABX}, J_{\mathrm{AB}}=9.2 \mathrm{~Hz}, J_{\mathrm{AX}}=3.6 \mathrm{~Hz}, 1 \mathrm{H}\right), 3.11\left(\mathrm{dd}, \mathrm{B}\right.$ of $\mathrm{ABX}, J_{\mathrm{AB}}=9.2 \mathrm{~Hz}$, $\left.J_{\mathrm{BX}}=7.6 \mathrm{~Hz}, 1 \mathrm{H}\right), 2.38(\mathrm{td}, J=7.6,2.8 \mathrm{~Hz}, 2 \mathrm{H}), 1.69(\mathrm{~s}, 3 \mathrm{H}), 1.42$ (quint, $J=7.6 \mathrm{~Hz}, 2 \mathrm{H}), 1.23(\mathrm{sext}, J=7.6 \mathrm{~Hz}, 2 \mathrm{H}), 0.78(\mathrm{t}, J=7.6 \mathrm{~Hz}$, $3 \mathrm{H}) ;{ }^{13} \mathrm{C}\{1 \mathrm{H}\} \mathrm{NMR}\left(100 \mathrm{MHz}, \mathrm{CDCl}_{3}\right) \delta: 213.6,167.6,137.7,128.5$, 128.4, 128.0, 127.9, 127.8, 103.2, 99.9, 74.0, 73.4, 71.2, 52.1, 30.1, 28.6, 22.2, 14.7, 13.9; FTIR $\left(\nu_{\max } / \mathrm{cm}^{-1}\right): 3416,2954,2928,2869,1943$, $1714,1454,1435,1379,1322,1266,1134,1016,965,738,698 . \mathrm{MS}$ (EI, $m / z)$ : $344\left(1, \mathrm{M}^{+}\right), 312(1), 279$ (3), 253 (2), 191 (2), 167 (20), 149 (77), 113 (10), 104 (12), 91 (65), 70 (35), 57 (42), 57 (54), 43 (80), 41 (100); HRMS (ESI): $\mathrm{C}_{21} \mathrm{H}_{29} \mathrm{O}_{4}(\mathrm{MH})^{+}, 345.2060$ (calculated); 345.2065 (found).

2da: Yield: $21.3 \mathrm{mg}(84 \%)$; ${ }^{1} \mathrm{H}$ NMR $\left(400 \mathrm{MHz}, \mathrm{C}_{6} \mathrm{D}_{6}\right): \delta: 6.35$ (d, $J=16.0 \mathrm{~Hz}, 1 \mathrm{H}$, major), $6.34(\mathrm{~d}, J=16.0 \mathrm{~Hz}, 1 \mathrm{H}$, minor), 5.48 (dd, $J=16.0,5.9 \mathrm{~Hz}, 1 \mathrm{H}), 4.10-4.04(\mathrm{~m}, 1 \mathrm{H}), 3.44-3.24(\mathrm{~m}, 2 \mathrm{H}), 3.37(\mathrm{~s}$, $3 \mathrm{H}), 2.39(\mathrm{td}, J=7.6,3.2 \mathrm{~Hz}, 2 \mathrm{H}), 1.70(\mathrm{~s}, 3 \mathrm{H}), 1.44$ (quint, $J=7.6$ $\mathrm{Hz}, 2 \mathrm{H}), 1.25$ (sext, $J=7.6 \mathrm{~Hz}, 2 \mathrm{H}), 0.80(\mathrm{t}, J=7.6 \mathrm{~Hz}, 3 \mathrm{H}$, major), $0.80(\mathrm{t}, J=7.2 \mathrm{~Hz}, 3 \mathrm{H}$, minor $) ;{ }^{13} \mathrm{C}\{1 \mathrm{H}\}$ NMR: $\left(100 \mathrm{MHz}, \mathrm{C}_{6} \mathrm{D}_{6}\right) \delta$ : 213.6, 167.0, 129.2, 128.0, 103.2, 100.1, 72.8, 66.3, 51.4, 30.3, 28.9, 22.2, 14.5, 13.7; FTIR $\left(\nu_{\max } / \mathrm{cm}^{-1}\right): 3384,2954,2928,2871,1940$, 1715, 1436, 1379, 1265, 1240, 1131, 1076, 1017, 965, 875; LC-MS/ MS: 255 (100, $\left.\mathrm{MH}^{+}\right), 237$ (20), 227 (8), 225 (20), 223 (42), 207 (12), 205 (50), 195 (12), 193 (15), 177 (18), 175 (18), 163 (15), 149 (8); HRMS (ESI): $\mathrm{C}_{14} \mathrm{H}_{23} \mathrm{O}_{4}(\mathrm{MH})^{+}, 255.1596$ (calculated); 255.1591 (found).

2ea: Yield: $30.2 \mathrm{mg}(82 \%) ;{ }^{1} \mathrm{H}$ NMR (400 MHz, $\left.\mathrm{C}_{6} \mathrm{D}_{6}\right) \delta: 6.45$ (dd, $J=15.8,1.6 \mathrm{~Hz}, 1 \mathrm{H}$, major), 6.34 (dd, $J=15.8,1.6 \mathrm{~Hz}, 1 \mathrm{H}$, minor) 5.54 (dd, $J=15.8,5.6 \mathrm{~Hz}, 1 \mathrm{H}$, major), $5.50(\mathrm{dd}, J=15.8,5.6 \mathrm{~Hz}, 1 \mathrm{H}$, minor), 4.19-4.10 (m, $1 \mathrm{H}), 3.42\left(\mathrm{dd}, \mathrm{A}\right.$ of $\mathrm{ABX}, J_{\mathrm{AB}}=9.6 \mathrm{~Hz}, J_{\mathrm{AX}}=$ $4.4 \mathrm{~Hz}, 1 \mathrm{H}), 3.37(\mathrm{~s}, 3 \mathrm{H}), 3.31\left(\mathrm{dd}, \mathrm{B}\right.$ of $\mathrm{ABX}, J_{\mathrm{AB}}=9.6 \mathrm{~Hz}, J_{\mathrm{BX}}=7.2$ $\mathrm{Hz}, 1 \mathrm{H}), 2.39(\mathrm{td}, J=7.2,1.6 \mathrm{~Hz}, 2 \mathrm{H}), 2.24(\mathrm{~d}, J=3.6 \mathrm{~Hz}, 1 \mathrm{H}), 1.72$ (s, 3H, major), 1.69 (s, 3H, minor), 1.44 (quint, $J=7.2 \mathrm{~Hz}, 2 \mathrm{H}$ ), 1.24 (sext, $J=7.2 \mathrm{~Hz}, 2 \mathrm{H}$ ), 0.89 (s, 9H, minor), 0.85 (s, 9H, major), 0.79 $(\mathrm{t}, J=7.2 \mathrm{~Hz}, 3 \mathrm{H}),-0.06(\mathrm{~s}, 6 \mathrm{H}) ;{ }^{13} \mathrm{C}\{1 \mathrm{H}\}$ NMR $\left(100 \mathrm{MHz}, \mathrm{C}_{6} \mathrm{D}_{6}\right)$ $\delta: 213.5,166.9,129.2,127.5,103.1,100.0,72.4,67.2,51.3,30.3,28.9$, 25.6, 22.2, 18.1, 14.5, 13.7, -5.6, -5.7; FTIR $\left(\nu_{\max } / \mathrm{cm}^{-1}\right): 3465,2954$, 2928, 2858, 1940, 1716, 1463, 1435, 1361, 1258, 1118, 963, 837, 777; MS (EI, $m / z): 368\left(1, \mathrm{M}^{+}\right), 339$ (1), 311 (2), 279 (1), 249 (3), 219 (2), 191 (4), 175 (5), 159 (6), 145 (3), 121 (5), 117 (15), 105 (30), 91 (20), 77 (20), 75 (100), 73 (50), 57 (85), 41 (60); HRMS (ESI): $\mathrm{C}_{20} \mathrm{H}_{37} \mathrm{O}_{4} \mathrm{Si}(\mathrm{MH})^{+}, 369.2456$ (calculated), 369.2470 (found).

2fa: Yield: $25.4 \mathrm{mg}(90 \%) ;{ }^{1} \mathrm{H}$ NMR $\left(400 \mathrm{MHz}, \mathrm{C}_{6} \mathrm{D}_{6}\right) \delta: 6.29(\mathrm{dd}$, $J=15.7,1.2 \mathrm{~Hz}, 1 \mathrm{H}$, minor), 6.28 (dd, $J=15.7,1.2 \mathrm{~Hz}, 1 \mathrm{H}$, major), $5.63(\mathrm{dd}, J=15.7,6.6 \mathrm{~Hz}, 1 \mathrm{H}$, minor), $5.62(\mathrm{dd}, J=15.7,6.6 \mathrm{~Hz}, 1 \mathrm{H}$, major), $3.73(\mathrm{~d}, J=6.6 \mathrm{~Hz}, 1 \mathrm{H}), 3.36(\mathrm{~s}, 3 \mathrm{H}$, major), $3.34(\mathrm{~s}, 3 \mathrm{H}$, minor), 2.38 (td, $J=7.2,1.6 \mathrm{~Hz}, 2 \mathrm{H}), 2.15(\mathrm{bs}, 1 \mathrm{H}), 2.00(\mathrm{bs}, 1 \mathrm{H})$, 1.71 (s, 3H, minor), $1.71(\mathrm{~s}, 3 \mathrm{H}$, major), 1.43 (quint, $J=7.2 \mathrm{~Hz}, 2 \mathrm{H}$ ), 1.23 (sext, $J=7.2 \mathrm{~Hz}, 2 \mathrm{H}), 1.06$ (s, $3 \mathrm{H}$, major), 1.05 (s, $3 \mathrm{H}$, minor), $1.03(\mathrm{~s}, 3 \mathrm{H}$, minor), $1.02(\mathrm{~s}, 3 \mathrm{H}$, major), $0.78(\mathrm{t}, J=7.2 \mathrm{~Hz}, 3 \mathrm{H})$; ${ }^{13} \mathrm{C}\{1 \mathrm{H}\}$ NMR $\left(100 \mathrm{MHz}, \mathrm{C}_{6} \mathrm{D}_{6}\right) \delta: 213.5,166.9,129.3,128.4,103.2$, 100.0, 79.2, 72.3, 51.3, 30.3, 28.8, 26.1, 23.8, 22.1, 14.5, 13.6; FTIR $\left(\nu_{\max } / \mathrm{cm}^{-1}\right): 3407,2956,2929,2860,1940,1714,1436,1377,1265$, 1242, 1133, 1015, 965, 898; MS (EI, $m / z): 282\left(2, \mathrm{M}^{+}\right), 250(2), 222$ (1), 191 (3), 164 (4), 134 (4), 121 (20), 91 (7), 77 (16), 65 (5), 59 (100), 43 (37); HRMS (ESI): $\mathrm{C}_{16} \mathrm{H}_{27} \mathrm{O}_{4}(\mathrm{MH})^{+}: 283.1904$ (calculated), 283.1908 (found).

2ga: Yield: $26.3 \mathrm{mg}$ (89\%); ${ }^{1} \mathrm{H}$ NMR $\left(400 \mathrm{MHz}, \mathrm{C}_{6} \mathrm{D}_{6}\right) \delta: 6.45$ (dd, $J=15.8,1.6 \mathrm{~Hz}, 1 \mathrm{H}), 5.68(\mathrm{dd}, J=15.8,6.2 \mathrm{~Hz}, 1 \mathrm{H}), 3.96(\mathrm{~d}, J=6.2$ $\mathrm{Hz}, 1 \mathrm{H}$ ), 3.36 (s, 3H, major), 3.33 (s, 3H, minor), 2.86 (s, 3H), 2.44 (bs, $1 \mathrm{H}), 2.38(\mathrm{td}, J=7.6,1.6 \mathrm{~Hz}, 2 \mathrm{H}), 1.72(\mathrm{~s}, 3 \mathrm{H}), 1.43$ (quint, $J=$ $7.6 \mathrm{~Hz}, 2 \mathrm{H}), 1.23(\mathrm{sext}, J=7.6 \mathrm{~Hz}, 2 \mathrm{H}), 0.93(\mathrm{~s}, 3 \mathrm{H}), 0.88(\mathrm{~s}, 3 \mathrm{H})$, $0.78(\mathrm{t}, J=7.6 \mathrm{~Hz}, 3 \mathrm{H}) ;{ }^{13} \mathrm{C}\{1 \mathrm{H}\}$ NMR $\left(100 \mathrm{MHz}, \mathrm{C}_{6} \mathrm{D}_{6}\right) \delta: 213.4$, 166.9, 129.1, 103.2, 99.9, 77.6, 77.1, 51.3, 48.6, 30.3, 28.9, 22.1, 20.2, 19.2, 14.6, 13.7; FTIR $\left(\nu_{\max } / \mathrm{cm}^{-1}\right): 3446,2953,2928,2859,1939$, 
$1712,1459,1435,1380,1321,1264,1241,1118,1062,1015,965,861$, 833, 740, 713; MS (EI, $m / z): 296\left(1, \mathrm{M}^{+}\right), 265$ (1), 233 (1), 221 (1), 191 (1), 121 (3), 91 (5), 74 (4), 73 (100), 59 (5), 43 (20); HRMS (ESI): $\mathrm{C}_{17} \mathrm{H}_{29} \mathrm{O}_{4}(\mathrm{MH})^{+}: 297.2060$ (calculated), 297.2063 (found).

2ha: Yield: $22.1 \mathrm{mg}(87 \%):{ }^{1} \mathrm{H}$ NMR $\left(400 \mathrm{MHz}, \mathrm{C}_{6} \mathrm{D}_{6}\right) \delta: 6.24$ (ddd, $J=15.2,10.6,1.6 \mathrm{~Hz}, 1 \mathrm{H}), 5.98(\mathrm{dt}, J=10.6,2.7 \mathrm{~Hz}, 1 \mathrm{H}), 5.46$ (ddd, $J=15.2,4.8,0.8 \mathrm{~Hz}, 1 \mathrm{H}), 4.16-4.08(\mathrm{~m}, 1 \mathrm{H}), 3.36(\mathrm{~s}, 3 \mathrm{H}$, major), 3.35 (s, 3H, minor), $2.99\left(\mathrm{dd}, \mathrm{A}\right.$ of $\mathrm{ABX}, J_{\mathrm{AB}}=9.6 \mathrm{~Hz}, J_{\mathrm{AX}}=$ $4.0 \mathrm{~Hz}, 1 \mathrm{H}), 2.93\left(\mathrm{dd}, \mathrm{B}\right.$ of $\left.\mathrm{ABX}, J_{\mathrm{AB}}=9.6 \mathrm{~Hz}, J_{\mathrm{BX}}=7.6 \mathrm{~Hz}, 1 \mathrm{H}\right), 2.93$ $(\mathrm{s}, 3 \mathrm{H}), 2.41-2.29(\mathrm{~m}, 2 \mathrm{H}), 2.20(\mathrm{bs}, 1 \mathrm{H}), 1.42$ (quint, $J=7.2 \mathrm{~Hz}$, $2 \mathrm{H}), 1.23$ (sext, $J=7.2 \mathrm{~Hz}, 2 \mathrm{H}), 0.79(\mathrm{t}, J=7.2 \mathrm{~Hz}, 3 \mathrm{H}$, minor), 0.78 $(\mathrm{t}, J=7.2 \mathrm{~Hz}, 3 \mathrm{H}$, major $) ;{ }^{13} \mathrm{C}\{1 \mathrm{H}\}$ NMR $\left(100 \mathrm{MHz}, \mathrm{C}_{6} \mathrm{D}_{6}\right) \delta: 213.8$, 166.6, 132.7, 124.1, 101.9, 97.0, 76.3, 70.3, 58.2, 51.3, 30.2, 28.6, 22.1, 13.6. FTIR $\left(\nu_{\max } / \mathrm{cm}^{-1}\right): 3423,2955,2930,2872,1941,1716,1436$, $1322,1268,1133,1062,967,853,713$; MS (EI, $m / z): 254\left(1, \mathrm{M}^{+}\right)$, 223 (1), 209 (2), 177 (4), 149 (3), 135 (4), 125 (6), 107 (8), 91 (7), 77 (10), 65 (5), 59 (10), 45 (100); HRMS (ESI): $\mathrm{C}_{14} \mathrm{H}_{23} \mathrm{O}_{4}(\mathrm{M})^{+}$: 255.1591 (calculated), 255.1593 (found).

2ia: Yield: $26.4 \mathrm{mg}(82 \%) ;{ }^{1} \mathrm{H}$ NMR $\left(400 \mathrm{MHz}, \mathrm{CDCl}_{3}\right) \delta: 6.08(\mathrm{~d}$, $J=15.8 \mathrm{~Hz}, 1 \mathrm{H}), 5.72(\mathrm{dd}, J=15.8,6.8 \mathrm{~Hz}, 1 \mathrm{H}), 4.16(\mathrm{q}, J=6.8 \mathrm{~Hz}$, $1 \mathrm{H}), 3.70(\mathrm{~s}, 3 \mathrm{H}), 2.31-2.15(\mathrm{~m}, 4 \mathrm{H}), 1.62-1.28(\mathrm{~m}, 16 \mathrm{H}), 0.93(\mathrm{~m}$, $9 \mathrm{H}) ;{ }^{13} \mathrm{C}\{1 \mathrm{H}\}$ NMR: $\left(100 \mathrm{MHz}, \mathrm{CDCl}_{3}\right) \delta: 213.1,167.8,132.8$, 126.2, 108.2, 101.4, 73.1, 52.0, 37.0, 30.3, 29.6, 28.7, 28.3, 27.6, 22.6, 22.4, 22.3, 14.0, 13.9, 13.8; FTIR $\left(\nu_{\max } / \mathrm{cm}^{-1}\right)$ : 3418, 2956, 2929, 2860, 1937, 1716, 1465, 1435, 1378, 1265, 1133, 1072, 964; MS (EI, $m / z): 322\left(1, \mathrm{M}^{+}\right), 236(3), 194$ (5), 163 (5), 119 (5), 105 (6), 91 (17), 85 (12), 69 (30), 57 (50), 41 (100); HRMS (ESI): $\mathrm{C}_{20} \mathrm{H}_{35} \mathrm{O}_{3}$ $(\mathrm{MH})^{+}: 323.2581$ (calculated), 323.2584 (found).

2ia': Yield: $25.5 \mathrm{mg}(80 \%) ;{ }^{1} \mathrm{H}$ NMR (400 $\left.\mathrm{MHz}, \mathrm{CDCl}_{3}\right) \delta: 6.08$ $(\mathrm{d}, J=15.7 \mathrm{~Hz}, 1 \mathrm{H}), 5.72(\mathrm{dd}, J=15.7,6.7 \mathrm{~Hz}, 1 \mathrm{H}), 4.17(\mathrm{q}, J=6.7$ $\mathrm{Hz}, 1 \mathrm{H}), 3.71(\mathrm{~s}, 3 \mathrm{H}), 2.30-2.14(\mathrm{~m}, 4 \mathrm{H}), 1.71-1.18(\mathrm{~m}, 15 \mathrm{H})$, $0.97-0.82(\mathrm{~m}, 9 \mathrm{H}) ;{ }^{13} \mathrm{C}\{1 \mathrm{H}\}$ NMR $\left(100 \mathrm{MHz}, \mathrm{CDCl}_{3}\right) \delta: 213.1$, 167.8, 132.8, 126.1, 108.2, 101.4, 73.0, 52.0, 37.1, 30.4, 29.6, 28.7, 28.4, 27.6, 22.6, 22.4, 22.3, 14.0, 13.9; FTIR $\left(\nu_{\max } / \mathrm{cm}^{-1}\right): 3422,2956,2928$, 2859, 1937, 1716, 1465, 1435, 1379, 1264, 1132, 1074, 963; MS (EI, $m / z): 322\left(1, \mathrm{M}^{+}\right), 236(20), 231$ (21), 205 (20), 195 (36), 194 (90), 179 (35), 177 (42), 165 (39), 163 (50), 152 (38), 135 (55), 121 (61),107 (68), 105 (60), 93(72), 91 (74), 85 (69), 79 (62), 57 (100), 55 (41); HRMS (ESI) $\mathrm{C}_{20} \mathrm{H}_{35} \mathrm{O}_{3}(\mathrm{MH})^{+}: 323.2581$ (calculated), 323.2584 (found).

2ja: Yield: $20.7 \mathrm{mg}(87 \%)$; ${ }^{1} \mathrm{H}$ NMR $\left(400 \mathrm{MHz}, \mathrm{C}_{6} \mathrm{D}_{6}\right) \delta: 6.16(\mathrm{dd}$, $J=15.6,1.2 \mathrm{~Hz}, 1 \mathrm{H}), 5.50(\mathrm{dd}, J=15.6,6.2 \mathrm{~Hz}, 1 \mathrm{H}$, minor), 5.49 (dd, $J=15.6,6.2 \mathrm{~Hz}, 1 \mathrm{H}$, major), 4.03 (quint, $J=6.2 \mathrm{~Hz}, 1 \mathrm{H}), 3.38(\mathrm{~s}, 3 \mathrm{H}$, major), $3.37(\mathrm{~s}, 3 \mathrm{H}$, minor $), 2.39(\mathrm{td}, J=7.4,1.2 \mathrm{~Hz}, 2 \mathrm{H}), 1.70(\mathrm{~s}$, $3 \mathrm{H}), 1.49-1.41(\mathrm{~m}, 2 \mathrm{H}), 1.25$ (sext, $J=7.4 \mathrm{~Hz}, 2 \mathrm{H}), 1.04(\mathrm{~d}, J=6.2$ $\mathrm{Hz}, 3 \mathrm{H}), 0.80(\mathrm{t}, J=7.4 \mathrm{~Hz}, 3 \mathrm{H}$, minor $), 0.80(\mathrm{t}, J=7.4 \mathrm{~Hz}, 3 \mathrm{H}$, major); ${ }^{13} \mathrm{C}\{1 \mathrm{H}\}$ NMR (100 MHz, $\left.\mathrm{C}_{6} \mathrm{D}_{6}\right) \delta: 213.3,166.9,134.7$, 125.2, 103.1, 99.9, 68.0, 51.2, 30.3, 28.9, 23.1, 22.1, 14.5, 13.6; FTIR $\left(\nu_{\max } / \mathrm{cm}^{-1}\right): 3410,2956,2928,1941,1715,1436,1322,1266,1135$, 1062, 1016, 965, 714; MS (EI, $m / z): 238\left(3, \mathrm{M}^{+}\right), 205(5), 194,(15)$, 181 (15), 164 (13), 151 (55), 135 (45), 121 (100), 107 (30), 93 (90), 77 (45), 65 (10), 55 (14), 43 (92); HRMS (ESI): $\mathrm{C}_{14} \mathrm{H}_{23} \mathrm{O}_{3}(\mathrm{MH})^{+}$: 239.1648 (calculated), 239.1652 (found).

2ka: Yield: $29.9 \mathrm{mg}$ (89\%): ${ }^{1} \mathrm{H}$ NMR (400 MHz, $\left.\mathrm{C}_{6} \mathrm{D}_{6}\right) \delta: 6.27$ (dd, $J=15.8,1.4 \mathrm{~Hz}, 1 \mathrm{H}$, major), $6.26(\mathrm{dd}, J=15.8,1.4 \mathrm{~Hz}, 1 \mathrm{H}$, minor), $5.78(\mathrm{dd}, J=15.8,5.5 \mathrm{~Hz}, 1 \mathrm{H}), 4.27-4.23(\mathrm{~m}, 1 \mathrm{H}), 3.38(\mathrm{~s}, 3 \mathrm{H}$, major), 3.37 (s, $3 \mathrm{H}$, minor), 3.05 (dd, $\mathrm{A}$ of $\mathrm{ABX}, J_{\mathrm{AB}}=9.6 \mathrm{~Hz}, J_{\mathrm{AX}}=$ $4.0 \mathrm{~Hz}, 1 \mathrm{H}), 3.02\left(\mathrm{dd}, \mathrm{B}\right.$ of $\left.\mathrm{ABX}, J_{\mathrm{AB}}=9.6 \mathrm{~Hz}, J_{\mathrm{BX}}=7.2 \mathrm{~Hz}, 1 \mathrm{H}\right), 2.94$ (s, 3H), 2.46-2.41 (m, 2H), $2.34(\mathrm{bs}, 1 \mathrm{H}), 2.16-2.09(\mathrm{~m}, 1 \mathrm{H}), 2.03-$ $1.99(\mathrm{~m}, 1 \mathrm{H}), 1.92-1.87(\mathrm{~m}, 1 \mathrm{H}), 1.66-1.60(\mathrm{~m}, 2 \mathrm{H}), 1.54-1.45(\mathrm{~m}$, $3 \mathrm{H}), 1.33-1.01(\mathrm{~m}, 7 \mathrm{H}), 0.81(\mathrm{t}, J=7.2 \mathrm{~Hz}, 3 \mathrm{H}$, major $), 0.81(\mathrm{t}, J=$ $7.2 \mathrm{~Hz}, 3 \mathrm{H}$, minor); ${ }^{13} \mathrm{C}$ NMR $\left(100 \mathrm{MHz}, \mathrm{C}_{6} \mathrm{D}_{6}\right) \delta: 212.3,167.1$, 129.0, 125.9, 113.8, 102.7, 76.5, 70.8, 58.2, 51.3, 38.0, 32.8, 32.4, 30.6, 29.0, 26.4, 26.4, 26.2, 22.4, 13.7; FTIR $\left(\nu_{\max } / \mathrm{cm}^{-1}\right): 3426,2925,2853$, $1924,1713,1435,1322,1263,1240,1195,1173,1119,1062,1016$, 964, 890, 841, 713; MS (EI, $m / z): 336\left(2, \mathrm{M}^{+}\right), 291(60), 273(8), 259$ (100), 241 (20), 231 (30), 213 (27), 203 (30), 189 (19), 175 (21), 161 (22), 145 (21), 133 (21), 119 (19), 105 (25), 91(47), 79 (33), 67 (21), 55 (45), 45 (65); HRMS (ESI): $\mathrm{C}_{20} \mathrm{H}_{33} \mathrm{O}_{4}(\mathrm{MH})^{+}: 337.2380$ (calculated), 337.2384 (found). 2la: Yield: $21.1 \mathrm{mg}(68 \%) ;{ }^{1} \mathrm{H}$ NMR $\left(400 \mathrm{MHz}, \mathrm{C}_{6} \mathrm{D}_{6}\right) \delta: 6.22$ (dd, $J=15.6,1.6 \mathrm{~Hz}, 1 \mathrm{H}$, major), 6.21 (dd, $J=15.6,1.6 \mathrm{~Hz}, 1 \mathrm{H}$, minor), $5.92(\mathrm{dd}, J=15.6,5.2 \mathrm{~Hz}, 1 \mathrm{H}), 4.23-4.15(\mathrm{~m}, 1 \mathrm{H}), 3.38(\mathrm{~s}, 3 \mathrm{H}), 3.01$ (dd, $\mathrm{A}$ of $\left.\mathrm{ABX}, J_{\mathrm{AB}}=9.2 \mathrm{~Hz}, J_{\mathrm{AX}}=3.6 \mathrm{~Hz}, 1 \mathrm{H}\right), 2.95$ (dd, B of $\mathrm{ABX}$, $\left.J_{\mathrm{AB}}=9.2 \mathrm{~Hz}, J_{\mathrm{BX}}=7.6 \mathrm{~Hz}, 1 \mathrm{H}\right), 2.92(\mathrm{~s}, 3 \mathrm{H}), 2.44-2.37(\mathrm{~m}, 2 \mathrm{H}), 2.29$ (bs, $1 \mathrm{H}), 1.51-1.46(\mathrm{~m}, 2 \mathrm{H}), 1.26(\mathrm{sext}, J=7.2 \mathrm{~Hz}, 2 \mathrm{H}), 1.07(\mathrm{~s}, 9 \mathrm{H})$, $0.81(\mathrm{t}, J=7.2 \mathrm{~Hz}, 3 \mathrm{H}) ;{ }^{13} \mathrm{C}\{1 \mathrm{H}\}$ NMR $\left(100 \mathrm{MHz}, \mathrm{C}_{6} \mathrm{D}_{6}\right) \delta: 208.6$, 167.4, 132.0, 123.6, 117.1, 102.7, 76.4, 70.6, 58.2, 51.3, 33.8, 30.5, 30.1, 28.9, 22.4, 13.7; FTIR $\left(\nu_{\max } / \mathrm{cm}^{-1}\right): 3455,2957,2929,2871,1940$, $1713,1459,1435,1395,1363,1322,1264,1239,1195,1172,1132$, 1062, 1017, 963, 713; LC-MS/MS: $311\left(67, \mathrm{MH}^{+}\right), 292$ (19), 279 (80), 261 (27), 255 (10), 251 (5), 247 (10), 237 (9), 229 (60), 223 (100), 219 (8), 205 (8), 201 (9), 191 (8), 179 (26), 177 (8), 169 (10); HRMS (ESI): $\mathrm{C}_{18} \mathrm{H}_{31} \mathrm{O}_{4}(\mathrm{MH})^{+}: 311.2223$ (calculated), 311.2220 (found).

2ma: Yield: $17.2 \mathrm{mg}(81 \%) ;{ }^{1} \mathrm{H}$ NMR $\left(400 \mathrm{MHz}, \mathrm{C}_{6} \mathrm{D}_{6}\right) \delta: 6.29(\mathrm{~d}$, $J=16.0 \mathrm{~Hz}, 1 \mathrm{H}$, major), $6.26(\mathrm{~d}, J=15.9 \mathrm{~Hz}, 1 \mathrm{H}$, minor), 5.69-5.66 (m, 1H), 5.47 (ddd, $J=15.9,5.2,1.2 \mathrm{~Hz}, 1 \mathrm{H}), 4.17-4.12(\mathrm{~m}, 1 \mathrm{H})$, 3.32 (s, 3H, major), 3.31 (s, 3H, minor), 3.00 (dd, $\mathrm{A}$ of $\mathrm{ABX}, J_{\mathrm{AB}}=9.6$ $\left.\mathrm{Hz}, J_{\mathrm{AX}}=4.0 \mathrm{~Hz}, 1 \mathrm{H}\right), 2.95\left(\mathrm{dd}, \mathrm{B}\right.$ of $\mathrm{ABX}, J_{\mathrm{AB}}=9.6 \mathrm{~Hz}, J_{\mathrm{BX}}=7.2 \mathrm{~Hz}$, $1 \mathrm{H}), 2.95(\mathrm{~s}, 3 \mathrm{H}), 2.08(\mathrm{bs}, 1 \mathrm{H}), 1.55(\mathrm{~d}, J=2.7 \mathrm{~Hz}, 3 \mathrm{H}) ;{ }^{13} \mathrm{C}\{1 \mathrm{H}\}$ NMR $\left(100 \mathrm{MHz}, \mathrm{C}_{6} \mathrm{D}_{6}\right) \delta: 216.1,165.0,130.2,126.0,103.5,87.5$, 76.3, 70.5, 58.2, 51.1, 14.0; FTIR $\left(\nu_{\max } / \mathrm{cm}^{-1}\right): 3429,2925,1944$ $1721,1438,1395,1260,1196,1153,1126,1028,966,840$; MS (EI, $m / z): 212\left(2, \mathrm{M}^{+}\right), 193(1), 167$ (3), 135 (6), 110 (6), 107 (11), 81 (5), 77 (20), 67 (6), 59 (11), 45 (100); HRMS (ESI): $\mathrm{C}_{11} \mathrm{H}_{17} \mathrm{O}_{4}$ $\left(\mathrm{MH}^{+}\right): 213.1121$ (calculated), 213.1120 (found).

2na: Yield: $27.0 \mathrm{mg}(92 \%) ;{ }^{1} \mathrm{H}$ NMR $\left(400 \mathrm{MHz}, \mathrm{C}_{6} \mathrm{D}_{6}\right) \delta: 6.46$ (dd, $J=15.7,1.6 \mathrm{~Hz}, 1 \mathrm{H}), 5.53(\mathrm{dd}, J=15.7,5.5 \mathrm{~Hz}, 1 \mathrm{H}), 4.27-4.20$ (m, $1 \mathrm{H}), 3.36$ (s, 3H, major), 3.35 (s, $3 \mathrm{H}$, minor), 3.05 (dd, A of ABX, $\left.J_{\mathrm{AB}}=9.6 \mathrm{~Hz}, J_{\mathrm{AX}}=4.0 \mathrm{~Hz}, 1 \mathrm{H}\right), 3.00\left(\mathrm{dd}, \mathrm{B}\right.$ of $\mathrm{ABX}, J_{\mathrm{AB}}=9.6 \mathrm{~Hz}, J_{\mathrm{BX}}=$ $7.2 \mathrm{~Hz}, 1 \mathrm{H}), 2.96(\mathrm{~s}, 3 \mathrm{H}), 2.64(\mathrm{tt}, J=11.4,3.4 \mathrm{~Hz}, 1 \mathrm{H}), 2.33(\mathrm{bs}$, $1 \mathrm{H}), 1.95(\mathrm{~d}, J=11.4 \mathrm{~Hz}, 2 \mathrm{H}), 1.71(\mathrm{~s}, 3 \mathrm{H}), 1.64-1.55(\mathrm{~m}, 2 \mathrm{H})$, $1.53-1.45(\mathrm{~m}, 1 \mathrm{H}), 1.30-0.94(\mathrm{~m}, 5 \mathrm{H}) ;{ }^{13} \mathrm{C}$ NMR $\left(100 \mathrm{MHz}, \mathrm{C}_{6} \mathrm{D}_{6}\right)$ $\delta: 213.2,166.6,129.1,105.9,104.3,76.5,70.6,58.2,51.2,37.6,32.8$, 26.4, 26.3, 26.0, 14.6; FTIR $\left(\nu_{\max } / \mathrm{cm}^{-1}\right): 3420,2924,2851,1936$, $1712,1435,1369,1322,1254,1228,1192,1128,1093,1071,1026$, 997, 965, 891, 872, 780; MS (EI, $m / z): 294\left(2, \mathrm{M}^{+}\right), 262(1), 249$ (2), 217 (3), 189 (1), 171 (4), 135 (2), 119 (3), 105 (5), 91 (6), 77 (6), 67 (4), 55 (8), 45 (100); HRMS (ESI): $\mathrm{C}_{17} \mathrm{H}_{27} \mathrm{O}_{4}(\mathrm{MH})^{+}: 295.1904$ (calculated), 295.1908 (found).

2oa: Yield: $23.9 \mathrm{mg}(89 \%) ;{ }^{1} \mathrm{H}$ NMR (400 MHz, $\left.\mathrm{C}_{6} \mathrm{D}_{6}\right) \delta: 6.43$ $(\mathrm{dd}, J=15.8,1.6 \mathrm{~Hz}, 1 \mathrm{H}), 5.51(\mathrm{dd}, J=15.8,5.6 \mathrm{~Hz}, 1 \mathrm{H}), 4.25-4.20$ (m, $1 \mathrm{H}), 3.32$ (s, 3H, major), 3.30 (s, $3 \mathrm{H}$, minor), 3.04 (dd, A of $\mathrm{ABX}$, $\left.J_{\mathrm{AB}}=9.6 \mathrm{~Hz}, J_{\mathrm{AX}}=4.4 \mathrm{~Hz}, 1 \mathrm{H}\right), 2.99\left(\mathrm{dd}, \mathrm{B}\right.$ of $\mathrm{ABX}, J_{\mathrm{AB}}=9.6 \mathrm{~Hz}, J_{\mathrm{BX}}=$ $7.2 \mathrm{~Hz}, 1 \mathrm{H}), 2.96(\mathrm{~s}, 3 \mathrm{H}), 2.34(\mathrm{bs}, 1 \mathrm{H}), 1.68(\mathrm{~s}, 3 \mathrm{H}), 1.29(\mathrm{~s}, 9 \mathrm{H})$; ${ }^{13} \mathrm{C}\{1 \mathrm{H}\} \mathrm{NMR}\left(100 \mathrm{MHz}, \mathrm{C}_{6} \mathrm{D}_{6}\right) \delta: 212.4,165.9,128.9,108.8,103.2$, 76.5, 70.7, 58.2, 50.9, 34.2, 29.3, 14.6; FTIR $\left(\nu_{\max } / \mathrm{cm}^{-1}\right): 3419,2955$, 2870, 1934, 1717, 1435, 1362, 1322, 1258, 1226, 1132, 1062, 965; MS (EI, $m / z): 268\left(1, \mathrm{M}^{+}\right), 236$ (1), 223 (2), 191 (3), 163 (5), 145 (5), 135 (15), 119 (5), 105 (6), 91 (12), 79 (15), 65 (5), 59 (7), 57 (25), 45 (100); HRMS (ESI): $\mathrm{C}_{15} \mathrm{H}_{25} \mathrm{O}_{4}(\mathrm{MH})^{+}: 269.1747$ (calculated), 269.1748 (found).

2ob: Yield: $25.7 \mathrm{mg}$ (91\%): ${ }^{1} \mathrm{H}$ NMR (400 MHz, $\left.\mathrm{C}_{6} \mathrm{D}_{6}\right) \delta: 6.44$ (dd, $J=15.5,1.6 \mathrm{~Hz}, 1 \mathrm{H}$, major), $6.42(\mathrm{dd}, J=15.5,1.6 \mathrm{~Hz}, 1 \mathrm{H}$, minor), $5.52(\mathrm{dd}, J=15.5,6.0 \mathrm{~Hz}, 1 \mathrm{H}), 4.23-4.19(\mathrm{~m}, 1 \mathrm{H}), 3.95(\mathrm{qd}$, $J=7.2 \mathrm{~Hz}, 1.2 \mathrm{~Hz}, 2 \mathrm{H}), 3.03\left(\mathrm{dd}, \mathrm{A}\right.$ of $\mathrm{ABX}, J_{\mathrm{AB}}=9.6 \mathrm{~Hz}, J_{\mathrm{AX}}=4.0$ $\mathrm{Hz}, 1 \mathrm{H}), 2.99\left(\mathrm{dd}, \mathrm{B}\right.$ of $\left.\mathrm{ABX}, J_{\mathrm{AB}}=9.6 \mathrm{~Hz}, J_{\mathrm{BX}}=7.2 \mathrm{~Hz}, 1 \mathrm{H}\right), 2.95(\mathrm{~s}$, $3 \mathrm{H}), 2.32(\mathrm{bs}, 1 \mathrm{H}), 1.70(\mathrm{~s}, 3 \mathrm{H}), 1.31(\mathrm{~s}, 9 \mathrm{H}), 0.89(\mathrm{t}, J=7.2 \mathrm{~Hz}$ $3 \mathrm{H}) ;{ }^{13} \mathrm{C}\{1 \mathrm{H}\} \mathrm{NMR}\left(100 \mathrm{MHz}, \mathrm{CDCl}_{3}\right) \delta: 212.1,166.2,129.0,127.4$, 109.2, 103.1, 76.5, 71.1, 60.3, 59.1, 34.2, 29.5, 14.8, 14.2; FTIR $\left(\nu_{\max } / \mathrm{cm}^{-1}\right): 3420,2959,1939,1713,1459,1366,1322,1255,1223$, 1112, 1060, 965; MS (EI, $m / z): 282\left(2, \mathrm{M}^{+}\right), 249$ (1), 237 (3), 208 (2), 191 (4), 163 (6), 135 (15), 105 (7), 91 (11), 77 (16), 65 (4), 57 (18), 45 (100); HRMS (ESI): $\mathrm{C}_{16} \mathrm{H}_{27} \mathrm{O}_{4}(\mathrm{MH})^{+} ; 283.1904$ (calculated), 283.1908 (found).

2oe: Yield: $26.0 \mathrm{mg}(88 \%){ }^{1} \mathrm{H}$ NMR $\left(400 \mathrm{MHz}, \mathrm{C}_{6} \mathrm{D}_{6}\right) \delta: 6.43(\mathrm{dd}$, $J=15.6,1.6 \mathrm{~Hz}, 1 \mathrm{H}), 5.53(\mathrm{dd}, J=15.6,5.6 \mathrm{~Hz}, 1 \mathrm{H}), 5.01$ (sept, $J=$ $6.4 \mathrm{~Hz}, 1 \mathrm{H}), 4.22-4.19(\mathrm{~m}, 1 \mathrm{H}), 3.03\left(\mathrm{dd}, \mathrm{A}\right.$ of $\mathrm{ABX}, J_{\mathrm{AB}}=9.6 \mathrm{~Hz}$, $\left.J_{\mathrm{AX}}=4.4 \mathrm{~Hz}, 1 \mathrm{H}\right), 2.99\left(\mathrm{dd}, \mathrm{B}\right.$ of $\left.\mathrm{ABX}, J_{\mathrm{AB}}=9.6 \mathrm{~Hz}, J_{\mathrm{BX}}=7.6 \mathrm{~Hz}, 1 \mathrm{H}\right)$, 
2.95 (s, 3H, major), 2.94 (s, 3H, minor), 2.26 (bs, 1H), 1.71 (s, 3H), $1.31(\mathrm{~s}, 9 \mathrm{H}), 1.00(\mathrm{~d}, J=6.4 \mathrm{~Hz}, 3 \mathrm{H}), 0.99(\mathrm{~d}, J=6.4 \mathrm{~Hz}, 3 \mathrm{H})$; ${ }^{13} \mathrm{C}\{1 \mathrm{H}\}$ NMR $\left(100 \mathrm{MHz}, \mathrm{CDCl}_{3}\right) \delta: 211.8,165.9,129.1,127.3$, 109.7, 103.1, 76.5, 71.1, 67.8, 59.0, 34.1, 29.5, 21.8, 21.8, 14.7; FTIR $\left(\nu_{\max } / \mathrm{cm}^{-1}\right): 3410,2960,2926,2896,2825,1935,1708,1458,1373$, 1322, 1255, 1226, 1049, 1021, 964, 833, 783, 713; MS (EI, $m / z): 296$ $\left(1, \mathrm{M}^{+}\right), 278$ (1), 251 (2), 236 (2), 191 (6), 163 (7), 135 (15), 105 (14), 91 (17), 77 (16), 65 (5), 57 (40), 45 (100); HRMS (ESI): $\mathrm{C}_{17} \mathrm{H}_{29} \mathrm{O}_{4}(\mathrm{MH})^{+}: 297.2060$ (calculated); 297.2064 (found).

2og: Yield: $25.9 \mathrm{mg}(88 \%) ;{ }^{1} \mathrm{H}$ NMR (400 MHz, $\left.\mathrm{C}_{6} \mathrm{D}_{6}\right) \delta: 6.42$ (dd, $J=15.6,1.6 \mathrm{~Hz}, 1 \mathrm{H}$, major), $6.40(\mathrm{dd}, J=15.6,1.6 \mathrm{~Hz}, 1 \mathrm{H}$, minor), 5.64 (ddt, $J=17.2,10.8,4.8 \mathrm{~Hz}, 1 \mathrm{H}$ ) $), 5.53(\mathrm{dd}, J=15.6,5.6$ $\mathrm{Hz}, 1 \mathrm{H}), 5.08(\mathrm{dq}, J=17.2,1.6 \mathrm{~Hz}, 1 \mathrm{H}), 4.87(\mathrm{dq}, J=10.8,1.6 \mathrm{~Hz}$, $1 \mathrm{H}), 4.48-4.37(\mathrm{~m}, 2 \mathrm{H}), 4.24-4.19(\mathrm{~m}, 1 \mathrm{H}), 3.03\left(\mathrm{dd}, \mathrm{A}\right.$ of $\mathrm{ABX}, J_{\mathrm{AB}}$ $\left.=9.6 \mathrm{~Hz}, J_{\mathrm{AX}}=4.0 \mathrm{~Hz}, 1 \mathrm{H}\right), 2.99\left(\mathrm{dd}, \mathrm{B}\right.$ of $\mathrm{ABX}, J_{\mathrm{AB}}=9.6 \mathrm{~Hz}, J_{\mathrm{BX}}=$ $7.2 \mathrm{~Hz}, 1 \mathrm{H}), 2.95$ (s, 3H, major), 2.94 (s, $3 \mathrm{H}$, minor), 2.28 (bs, $1 \mathrm{H})$, $1.69(\mathrm{~s}, 3 \mathrm{H}), 1.30(\mathrm{~s}, 9 \mathrm{H}) ;{ }^{13} \mathrm{C}\{1 \mathrm{H}\}$ NMR $\left(100 \mathrm{MHz} \mathrm{CDCl}_{3}\right) \delta$ : 212.4, 165.7, 132.5, 128.7, 127.7, 117.1, 108.9, 103.2, 76.5, 71.1, 64.7, 59.1, 34.2, 29.4, 14.8; FTIR $\left(\nu_{\max } / \mathrm{cm}^{-1}\right): 3410,2957,2927,1935$, $1713,1647,1457,1398,1362,1321,1252,1217,1172,1131,1061$, 1027, 1016, 964, 928, 836, 781, 713; MS (EI, $m / z): 294\left(1, \mathrm{M}^{+}\right), 249$ (3), 236 (2), 191 (4), 163 (5), 135 (6), 105 (5), 91 (10), 77 (9), 65 (3), 57 (16), 45 (100); HRMS (EI): $\mathrm{C}_{17} \mathrm{H}_{27} \mathrm{O}_{4}(\mathrm{MH})^{+}: 295.1904$ (calculated), 295.1907 (found).

2pa: Yield: $29.6 \mathrm{mg}(86 \%) ;{ }^{1} \mathrm{H}$ NMR (400 MHz, $\left.\mathrm{C}_{6} \mathrm{D}_{6}\right) \delta: 7.17-$ $7.05(\mathrm{~m}, 5 \mathrm{H}), 6.43(\mathrm{dd}, J=15.8,1.6 \mathrm{~Hz}, 1 \mathrm{H}), 5.49(\mathrm{dd}, J=15.8,5.6$ $\mathrm{Hz}, 1 \mathrm{H}), 4.28-4.24(\mathrm{~m}, 1 \mathrm{H}), 4.20$ (s, $2 \mathrm{H}$, major), 4.17 (s, 2H, minor), $3.31(\mathrm{~s}, 3 \mathrm{H}), 3.19\left(\mathrm{dd}, \mathrm{A}\right.$ of $\left.\mathrm{ABX}, J_{\mathrm{AB}}=9.2 \mathrm{~Hz}, J_{\mathrm{AX}}=3.6 \mathrm{~Hz}, 1 \mathrm{H}\right), 3.12$ $\left(\mathrm{dd}, \mathrm{B}\right.$ of $\left.\mathrm{ABX}, J_{\mathrm{AB}}=9.2 \mathrm{~Hz}, J_{\mathrm{BX}}=7.2 \mathrm{~Hz}, 1 \mathrm{H}\right), 2.32(\mathrm{bs}, 1 \mathrm{H}), 1.67(\mathrm{~s}$, $3 \mathrm{H}), 1.34$ (s, 9H, minor), 1.29 (s, 9H, major); ${ }^{13} \mathrm{C}\{1 \mathrm{H}\}$ NMR: $(100$ $\left.\mathrm{MHz}, \mathrm{CDCl}_{3}\right) \delta: 212.4,166.5,137.8,128.8,128.5,127.9,127.8,127.7$, 108.8, 103.1, 74.0, 73.4, 71.2, 51.6, 34.2, 29.4, 14.8; FTIR $\left(\nu_{\max } / \mathrm{cm}^{-1}\right)$ : $3422,2954,2865,1934,1715,1496,1479,1454,1434,1391,1362$, 1257, 1225, 1104, 1060, 1029, 965, 781, 737, 698; MS (EI, $m / z): 344$ $\left(2, \mathrm{M}^{+}\right), 311(2), 296$ (2), 223 (2), 191 (3), 168 (4), 135 (11), 107 (7), 91 (100), 57 (50), 41 (25); HRMS (ESI): $\mathrm{C}_{21} \mathrm{H}_{29} \mathrm{O}_{4}(\mathrm{MH})^{+}$: 345.2060 (calculated), 345.2068 (found).

2qa: Yield: $22.1 \mathrm{mg}(87 \%) ;{ }^{1} \mathrm{H}$ NMR (400 MHz, $\left.\mathrm{C}_{6} \mathrm{D}_{6}\right) \delta: 6.32$ (dd, $J=15.8,1.6 \mathrm{~Hz}, 1 \mathrm{H}$, major), $6.09(\mathrm{dd}, J=15.8,6.0 \mathrm{~Hz}, 1 \mathrm{H}$, minor), 5.74 (dd, $J=15.8,1.6 \mathrm{~Hz}, 1 \mathrm{H}$, minor), 5.43 (dd, $J=15.8,6.0$ $\mathrm{Hz}, 1 \mathrm{H}$, major), $4.03-3.99(\mathrm{~m}, 1 \mathrm{H}), 3.35$ (dd, $\mathrm{A}$ of $\mathrm{ABX}, J_{\mathrm{AB}}=11.2$ $\left.\mathrm{Hz}, J_{\mathrm{AX}}=3.6 \mathrm{~Hz}, 1 \mathrm{H}\right), 3.32(\mathrm{~s}, 3 \mathrm{H}), 3.23\left(\mathrm{dd}, \mathrm{B}\right.$ of $\mathrm{ABX}, J_{\mathrm{AB}}=11.2 \mathrm{~Hz}$, $\left.J_{\mathrm{BX}}=7.2 \mathrm{~Hz}, 1 \mathrm{H}\right), 2.44$ (bs, $\left.1 \mathrm{H}\right), 2.22(\mathrm{bs}, 1 \mathrm{H}), 1.67$ (s, 3H, major), 1.57 (s, 3H, minor), 1.34 (s, 9H, minor), 1.29 (s, 9H, major); ${ }^{13} \mathrm{C}$ NMR $\left(100 \mathrm{MHz}, \mathrm{C}_{6} \mathrm{D}_{6}\right) \delta: 212.4,165.9,128.9,108.9,103.2,72.8$, 66.3, 50.9, 34.1, 29.3, 14.6; NMR (100 MHz, $\left.\mathrm{CDCl}_{3}\right) \delta: 212.4,166.5$, 129.2, 127.9, 108.9, 103.1, 73.0, 66.4, 51.7, 34.2, 29.4, 14.8; FTIR $\left(\nu_{\max } / \mathrm{cm}^{-1}\right): 3368,2955,2926,2865,1934,1717,1641,1458,1434$, $1363,1257,1225,1060,1029,965,925,862,781 ;$ MS (EI, $m / z): 254$ $\left(3, \mathrm{M}^{+}\right): 207$ (7), 194 (11), 179 (10), 148 (13), 119 (15), 105 (20), 91 (35), 73 (20), 59 (20), 57 (100), 41 (50); HRMS (EI): $\mathrm{C}_{14} \mathrm{H}_{23} \mathrm{O}_{4}$ $(\mathrm{MH})^{+}: 255.1591$ (calculated), 255.1590 (found).

2ra: Yield: $30.9 \mathrm{mg},(84 \%):{ }^{1} \mathrm{H}$ NMR (400 MHz, $\left.\mathrm{C}_{6} \mathrm{D}_{6}\right) \delta: 6.43$ (dd, $J=15.6,1.6 \mathrm{~Hz}, 1 \mathrm{H}$, major), 6.41 (dd, $J=15.6,1.6 \mathrm{~Hz}, 1 \mathrm{H}$, minor), $5.53(\mathrm{dd}, J=15.6,5.6 \mathrm{~Hz}, 1 \mathrm{H}), 4.17-4.12(\mathrm{~m}, 1 \mathrm{H}), 3.42(\mathrm{dd}$, $\mathrm{A}$ of $\left.\mathrm{ABX}, J_{\mathrm{AB}}=10.0 \mathrm{~Hz}, J_{\mathrm{AX}}=4.0 \mathrm{~Hz}, 1 \mathrm{H}\right), 3.32\left(\mathrm{dd}, \mathrm{B}\right.$ of $\mathrm{ABX}, J_{\mathrm{AB}}=$ $\left.10.0 \mathrm{~Hz}, J_{\mathrm{BX}}=7.6 \mathrm{~Hz}, 1 \mathrm{H}\right), 3.32(\mathrm{~s}, 3 \mathrm{H}), 2.23(\mathrm{bs}, 1 \mathrm{H}), 1.71(\mathrm{~s}, 3 \mathrm{H})$, $1.29(\mathrm{~s}, 9 \mathrm{H}), 0.85(\mathrm{~s}, 9 \mathrm{H}),-0.06(\mathrm{~s}, 6 \mathrm{H}) ;{ }^{13} \mathrm{C}\{1 \mathrm{H}\} \mathrm{NMR}(100 \mathrm{MHz}$, $\left.\mathrm{C}_{6} \mathrm{D}_{6}\right) \delta: 212.4,165.9,129.1,108.8,103.2,72.5,67.2,50.9,34.1,29.3$, 25.6, 18.1, 14.6, -5.6, -5.7; FTIR $\left(\nu_{\max } / \mathrm{cm}^{-1}\right)$ : 3467, 2954, 2927, $2857,1935,1718,1462,1434,1362,1256,1225,1111,1062,1005$, 963, 837, 777; MS (EI, $m / z): 368\left(1, \mathrm{M}^{+}\right), 351$ (1), 311 (3), 279 (2), 237 (10), 223 (8), 191 (10), 177 (15), 163 (20), 159 (43), 145 (20), 135 (25), 105 (30), 89 (45), 75 (85), 73 (100), 57 (55), 41 (38); HRMS (ESI): $\mathrm{C}_{20} \mathrm{H}_{37} \mathrm{O}_{4} \mathrm{Si}(\mathrm{MH})^{+}: 369.2456$ (calculated), 369.2463 (found).

2sa: Yield: $25.1 \mathrm{mg}(87 \%) ;{ }^{1} \mathrm{H}$ NMR $\left(400 \mathrm{MHz}, \mathrm{C}_{6} \mathrm{D}_{6}\right) \delta: 7.71-$ $7.67(\mathrm{~m}, 2 \mathrm{H}), 7.14-7.11(\mathrm{~m}, 2 \mathrm{H}), 7.03-6.99(\mathrm{~m}, 1 \mathrm{H}), 6.41(\mathrm{dd}, J=$ $15.8,1.2 \mathrm{~Hz}, 1 \mathrm{H}$, major), 6.38 (dd, $J=15.8,1.2 \mathrm{~Hz}, 1 \mathrm{H}$, minor), 5.56 (dd, $J=15.8,5.6 \mathrm{~Hz}, 1 \mathrm{H}), 4.21-4.16(\mathrm{~m}, 1 \mathrm{H}), 3.38$ (s, 3H, major), $3.36(\mathrm{~s}, 3 \mathrm{H}, \mathrm{minor}), 3.03-2.96(\mathrm{~m}, 2 \mathrm{H}), 2.96(\mathrm{~s}, 3 \mathrm{H}), 2.30(\mathrm{bs}, 1 \mathrm{H})$, $1.68(\mathrm{~s}, 3 \mathrm{H}) ;{ }^{13} \mathrm{C}\{1 \mathrm{H}\}$ NMR $\left(100 \mathrm{MHz}, \mathrm{C}_{6} \mathrm{D}_{6}\right) \delta: 215.9,165.8,133.2$, $130.5,128.7,128.2,127.5,126.2,104.4,102.7,76.4,70.6,58.2,51.5$, 14.2; FTIR $\left(\nu_{\max } / \mathrm{cm}^{-1}\right): 3419,2922,2851,1926,1716,1492,1434$, 1369, 1321, 1273, 1195, 1171, 1123, 1062, 1039, 964, 918, 898, 781, 694; MS (EI, $m / z): 288\left(2, \mathrm{M}^{+}\right), 256$ (3), 211 (5), 183(5), 155 (17), 115 (8), 89 (4), 77 (9), 51 (5), 45 (100); HRMS (ESI): $\mathrm{C}_{17} \mathrm{H}_{21} \mathrm{O}_{4}$ $(\mathrm{MH})^{+}: 289.1434$ (calculated), 289.1439 (found).

2ta: Yield: $25.4 \mathrm{mg}(82 \%) ;{ }^{1} \mathrm{H}$ NMR $\left(400 \mathrm{MHz}, \mathrm{C}_{6} \mathrm{D}_{6}\right) \delta: 5.80(\mathrm{~d}$, $J=8.2,1.6 \mathrm{~Hz}, 1 \mathrm{H}$, minor), $5.75(\mathrm{dt}, J=8.2,1.6 \mathrm{~Hz}, 1 \mathrm{H}$, major $)$, $4.55-4.50(\mathrm{~m}, 1 \mathrm{H}), 3.36(\mathrm{~s}, 3 \mathrm{H}), 3.08-3.03(\mathrm{~m}, 2 \mathrm{H}), 2.96(\mathrm{~s}, 3 \mathrm{H})$, $2.40(\mathrm{td}, J=7.6,2.8 \mathrm{~Hz}, 2 \mathrm{H}), 2.25-2.11(\mathrm{~m}, 4 \mathrm{H}), 1.60-1.20(\mathrm{~m}, 4 \mathrm{H})$, $0.86-0.78(\mathrm{~m}, 5 \mathrm{H}) ;{ }^{13} \mathrm{C}\{1 \mathrm{H}\}$ NMR $\left(100 \mathrm{MHz}, \mathrm{C}_{6} \mathrm{D}_{6}\right) \delta: 207.1,167.4$, 137.2 , 125.6, 109.3, 99.6, 76.3, 66.9, 58.3, 51.4, 30.7, 30.5, 28.9, 28.5, 25.6, 25.5, 22.2, 13.9; FTIR $\left(\nu_{\max } / \mathrm{cm}^{-1}\right): 3456,2928,2857,2859$, 1946, 1713, 1436, 1436, 1266, 1241, 1128, 1063, 957; MS (EI, $\mathrm{m} / z)$ : $308\left(1, \mathrm{M}^{+}\right), 276$ (25), 231 (65), 201 (35), 175 (40), 161 (50), 117 (55), 91 (95), 77 (40), 55 (30), 45 (100); HRMS (ESI): $\mathrm{C}_{18} \mathrm{H}_{29} \mathrm{O}_{4}$ $(\mathrm{MH})^{+}: 310.2067$ (calculated), 310.2067 (found).

Carbamate Derivatization of $\mathbf{2 b a}$ and Crystal Growth for $\mathrm{X}$-ray Analysis. The mixture of vinylallene ester $(45.2 \mathrm{mg}, 0.2 \mathrm{mmol})$, phenyl isocyanate $(31 \mathrm{mg}, 0.26 \mathrm{mmol})$, and dry pyridine $(15.8 \mathrm{mg}$, $0.2 \mathrm{mmol}$ ) in $2 \mathrm{~mL}$ of toluene was stirred at room temperature for 5 days. The mixture was taken into $\mathrm{Et}_{2} \mathrm{O}$ and washed with brine, dried over $\mathrm{MgSO}_{4}$, and filtered. The solvent was removed in vacuum, and the crude residue was purified by column chromatography on silica gel (hexane-EtAc; 5:1, yield: $0.62 \mathrm{~g}, 80 \%){ }^{26}$ The pure white solid material was dissolved in a boiled hexane and gradually cooled down to $30^{\circ}$ overnight.

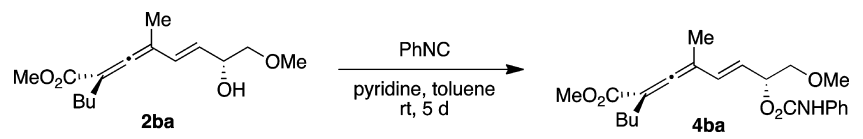

4ba: M.P.: $67-69{ }^{\circ} \mathrm{C} ;{ }^{1} \mathrm{H}$ NMR $\left(400 \mathrm{MHz}, \mathrm{CDCl}_{3}\right) \delta: 7.38(\mathrm{~d}, J=$ $7.6 \mathrm{~Hz}, 2 \mathrm{H}), 7.32-7.28(\mathrm{~m}, 2 \mathrm{H}), 7.06(\mathrm{tt}, J=7.6,1.2 \mathrm{~Hz}, 1 \mathrm{H}), 6.72$ (bs, $1 \mathrm{H}), 6.33(\mathrm{dd}, J=15.8,1.2 \mathrm{~Hz}, 1 \mathrm{H}), 5.70(\mathrm{dd}, J=15.8 \mathrm{~Hz}, 6.4$ $\mathrm{Hz}, 1 \mathrm{H}), 5.53(\mathrm{qd}, J=5.6,1.2 \mathrm{~Hz}, 1 \mathrm{H}), 3.70(\mathrm{~s}, 3 \mathrm{H}), 3.60(\mathrm{~d}, J=2.4$ $\mathrm{Hz}, 1 \mathrm{H}), 3.59(\mathrm{~d}, J=0.8 \mathrm{~Hz}, 1 \mathrm{H}), 3.42(\mathrm{~s}, 3 \mathrm{H}), 2.25(\mathrm{t}, J=7.6 \mathrm{~Hz}$, $2 \mathrm{H}), 1.89(\mathrm{~s}, 3 \mathrm{H}), 1.40-1.28(\mathrm{~m}, 4 \mathrm{H}), 0.88(\mathrm{t}, J=7.6 \mathrm{~Hz}, 3 \mathrm{H})$; ${ }^{13} \mathrm{C}\{1 \mathrm{H}\}$ NMR $\left(100 \mathrm{MHz}, \mathrm{CDCl}_{3}\right) \delta: 213.8,167.5,152.6,137.7$, 130.0, 129.1, 124.8, 123.5, 118.6, 103.0, 100.0, 74.1, 73.5, 59.3, 52.1, $30.1,28.6,22.1,14.7,13.9$.

\section{ASSOCIATED CONTENT}

\section{Supporting Information}

X-ray crystallographic data of $4 \mathrm{ba}$ and copies of ${ }^{1} \mathrm{H}$ NMR and ${ }^{13} \mathrm{C}$ NMR spectra for the substrates $\mathbf{1}$ and $\mathbf{1}^{\prime}$, the products 2 and $\mathbf{2}^{\prime}$, and $\mathbf{4 b a}$. The Supporting Information is available free of charge on the ACS Publications website at DOI: 10.1021/ acs.joc.5b00382.

\section{AUTHOR INFORMATION}

\section{Corresponding Author}

*E-mail: leventartok@iyte.edu.tr

Notes

The authors declare no competing financial interest.

\section{ACKNOWLEDGMENTS}

The financial support of the Scientific and Technological Research Council of Turkey and Federal Ministry of Education and Research (Germany) via the Intensified Cooperation Program (210T092) is gratefully acknowledged. We thank the Biological Mass and Proteomics Facility of Izmir Institute of Technology for MS/MS analyses and the Mass Spectrometry Laboratory of Dortmund University of Technology for HRMS analyses. 


\section{DEDICATION}

Dedicated to the memory of Prof. Dr. Ayhan S. Demir.

\section{REFERENCES}

(1) (a) Bertrand, M.; Grimaldi, J.; Waegel, B. Chem. Commun. (London) 1968, 1141-1142. (b) Baudouy, R.; Delbecq, F.; Gore, J. Tetrahedron 1980, 36, 189-195. (c) Schneider, R.; Siegel, H.; Hopf, H. Liebigs Ann. Chem. 1981, 1812-1825. (d) Deutsch, E. A.; Snider, B. B. J. Org. Chem. 1982, 47, 2682-2684. (e) Tolstikov, G. A.; Romanova, T. Y.; Kuchin, A. V. J. Organomet. Chem. 1985, 285, 7182. (f) Reich, H. J.; Eisenhart, E. K.; Whipple, W. L.; Kelly, M. J. J. Am. Chem. Soc. 1988, 110, 6432-6442. (g) Gibbs, R. A.; Bartels, K.; Lee, R. W. K.; Okamura, W. H. J. Am. Chem. Soc. 1989, 111, 3717-3725. (h) Wang, K. K.; Andemichael, Y. W.; Dhumrongvaraporn, S. Tetrahedron Lett. 1989, 30, 1311-1314. (i) Krause, N. Liebigs Ann. Chem. 1993, 521-525. (j) Koop, U.; Handke, G.; Krause, N. Liebigs Ann. 1996, 1487-1499. (k) Murakami, M.; Ubukata, M.; Itami, K.; Ito, Y. Angew. Chem., Int. Ed. 1998, 37, 2248-2250. (1) Murakami, M.; Itami, K.; Ito, Y. Organometallics 1999, 18, 1326-1336. (m) Murakami, M.; Itami, K.; Ito, Y. J. Am. Chem. Soc. 1999, 121, 4130-4135. (n) Regás, D.; Afonso, M. M.; Rodríguez, M. L.; Palenzuela, J. A. J. Org. Chem. 2003, 68, 7845-7852. (o) Murakami, M.; Ashida, S.; Matsuda, T. J. Am. Chem. Soc. 2004, 126, 10838-10839. (p) Lee, J. H.; Toste, F. D. Angew. Chem., Int. Ed. 2007, 46, 912-914. (q) Funami, H.; Kusama, H.; Iwasawa, N. Angew. Chem., Int. Ed. 2007, 46, 909911. (r) Yang, M.; Yokokawa, N.; Ohmiya, H.; Sawamura, M. Org. Lett. 2012, 14, 816-819.

(2) Bond, D. J. Org. Chem. 1990, 55, 661-665.

(3) (a) Gore, J.; Dulcere, J. P. J. Chem. Soc., Chem. Comm. 1972, 866-867. (b) Ruitenberg, K.; Kleijn, H.; Elsevier, C. J.; Meijer, J.; Vermeer, P. Tetrahedron Lett. 1981, 22, 1451-1452. (c) Keinan, E.; Bosch, E. J. Org. Chem. 1986, 51, 4006-4016. (d) Mandai, T.; Ogawa, M.; Yamaoki, H.; Nakata, T.; Murayama, H.; Kawada, M.; Tsuji, J. Tetrahedron Lett. 1991, 32, 3397-3398. (e) Purpura, M.; Krause, N. Eur. J. Org. Chem. 1999, 267-275. (f) Satoh, T.; Hanaki, N.; Kuramochi, Y.; Inoue, Y.; Hosoya, K.; Sakai, K. Tetrahedron 2002, 58 , 2533-2549. (g) Ogasawara, M.; Fan, L.; Ge, Y.; Takahashi, T. Org. Lett. 2006, 8, 5409-5412. (h) Molander, G. A.; Sommers, E. M.; Baker, S. R. J. Org. Chem. 2006, 71, 1563-1568. (i) Zhao, J.; Yu, Y.; Ma, S. Chem.-Eur. J. 2010, 16, 74-80. (j) Ye, J.; Li, S.; Ma, S. Org. Lett. 2012, 14, 2312-2315. (k) Dabrowski, J. A.; Haeffner, F.; Hoveyda, A. H. Angew. Chem., Int. Ed. 2013, 52, 7694-7699.

(4) (a) Akpınar, G. E.; Kuş, M.; Üçüncü, M.; Karakuş, E.; Artok, L. Org. Lett. 2011, 13, 748-751. (b) Üçüncü, M.; Karakuş, E.; Kuş, M.; Akpınar, G. E.; Aksın-Artok, Ö.; Krause, N.; Karaca, S.; Elmac1, N.; Artok, L. J. Org. Chem. 2011, 76, 5959-5971. (c) Karagöz, E. Ş.; Kuş, M.; Akpınar, G. E.; Artok, L. J. Org. Chem. 2014, 79, 9222-9230.

(5) CCDC 1019595 contains the supplementary crystallographic data of phenylcarbamate derivative of $\mathbf{2 b a}$. This data can be obtained free of charge from The Cambridge Crytallographic Data Centre via www.ccdc.cam.ac.uk/data_request/cif.

(6) Major diastereomeric products could usually be enriched by vigorous column purification. For instance, it has been possible to improve the $\mathrm{dr}$ of $2 \mathrm{ja}$ to $99: 1$ by column chromatography.

(7) The preparation of enyne oxiranes containing a phenyl group in the $R^{2}$ or $R^{3}$ positions failed owing to their decomposition during epoxidation process.

(8) Such a trend was also observed for the alkoxycarbonylation reactions of conjugated 2,4-enyne carbonates (ref 4c).

(9) The mechanism of this method is in keeping with those of analogue reactions of 2,4-enyne carbonates (ref 4c).

(10) (a) Trost, B. M.; Verhoeven, T. R. J. Org. Chem. 1976, 41, 3215. (b) Matsushita, H.; Negishi, E.-i. J. Chem. Soc., Chem. Commun. 1982, 160-161. (c) Hayashi, T.; Hagihara, T.; Konishi, M.; Kumada, M. J. Am. Chem. Soc. 1983, 105, 7767-7768. (d) Oshima, M.; Yamazaki, H.; Shimizu, I.; Nisar, M.; Tsuji, J. J. Am. Chem. Soc. 1989, 111, 62806287. (e) Shimizu, I.; Maruyama, T.; Makuta, T.; Yamamoto, A. Tetrahedron Lett. 1993, 34, 2135-2138.
(11) Hayashi, T.; Yamamoto, A.; Hagihara, T. J. Org. Chem. 1986, 51, 723-727.

(12) (a) Tsuji, J.; Sugiura, T.; Minami, I. Tetrahedron Lett. 1986, 27, 731-734. (b) Piotti, M. E.; Alper, H. J. Org. Chem. 1997, 62, 84848489. (c) Wang, Y.; Zhang, W.; Ma, S. J. Am. Chem. Soc. 2013, 135, 11517-11520.

(13) It has been reported that vinylallenes feature a strong tendency to enantiomerization (see ref $3 \mathrm{~h}$ ).

(14) Ukai, T.; Kawazura, H.; Ishii, Y.; Bonnet, J. J.; Ibers, J. A. J. Organomet. Chem. 1974, 65, 253-266.

(15) Wang, C.; Tobrman, T.; Xu, Z.; Negishi, E. Org. Lett. 2009, 11, 4092-4095.

(16) Stille, J. K.; Simpson, J. H. J. Am. Chem. Soc. 1987, 109, 21382152.

(17) Denmark, S. E.; Wang, Z. Org. Synth. 2005, 81, 42-52.

(18) Fang, Z.; Liao, P. C.; Yang, Y. L.; Yang, F. L.; Chen, Y. L.; Lam, Y.; Hua, Y. F.; Wu, S. H. J. Med. Chem. 2010, 53, 7967-7978.

(19) Ming-Yuan, L.; Madhushaw, R. J.; Liu, R. S. J. Org. Chem. 2004, 69, 7700-7704.

(20) Urabe, H.; Suzuki, K.; Sato, F. J. Am. Chem. Soc. 1997, 119, 10014-10027.

(21) Kajikawa, T.; Aoki, K.; Singh, R. S.; Iwashita, T.; Kusumoto, T.; Frank, H. A.; Hashimoto, H.; Katsumura, S. Org. Biomol. Chem. 2009, 7, 3723-3733.

(22) Caldentey, X. C.; Cambeiro, X. C.; Pericàs, M. Tetrahedron 2011, 67, 4161-4168.

(23) Schmidt, B.; Pohler, M.; Costisella, B. Tetrahedron 2002, 58, 7951-7958.

(24) Buss, A. D.; Warren, S. J. Chem. Soc., Perkin Trans. I 1985, $2307-2325$

(25) Lian, J. J.; Chiang, P. C.; Lin, Y. P.; Ting, H. C.; Liu, R. S. J. Am. Chem. Soc. 2006, 128, 11372-11373.

(26) Frigerio, M.; Santagostino, M.; Sputore, S.; Palmisano, G. J. Org. Chem. 1995, 60, 7272-7276. 